The copyright of this thesis vests in the author. No quotation from it or information derived from it is to be published without full acknowledgement of the source. The thesis is to be used for private study or noncommercial research purposes only.

Published by the University of Cape Town (UCT) in terms of the non-exclusive license granted to UCT by the author. 


\title{
A CFD study of fuel evaporation and related thermofluid dynamics in the inlet manifold, port and cylinder of the CFR octane engine
}

\author{
by
}

Anja Thiart

Thesis presented in partial fulfillment for the degree of

Master of Science in Engineering

Department of Mechanical Engineering

University of Cape Town

Supervised by

Dr. G. Floweday (SAFL) and Prof. C.M. Meyer (CERECAM)

January, 2012 


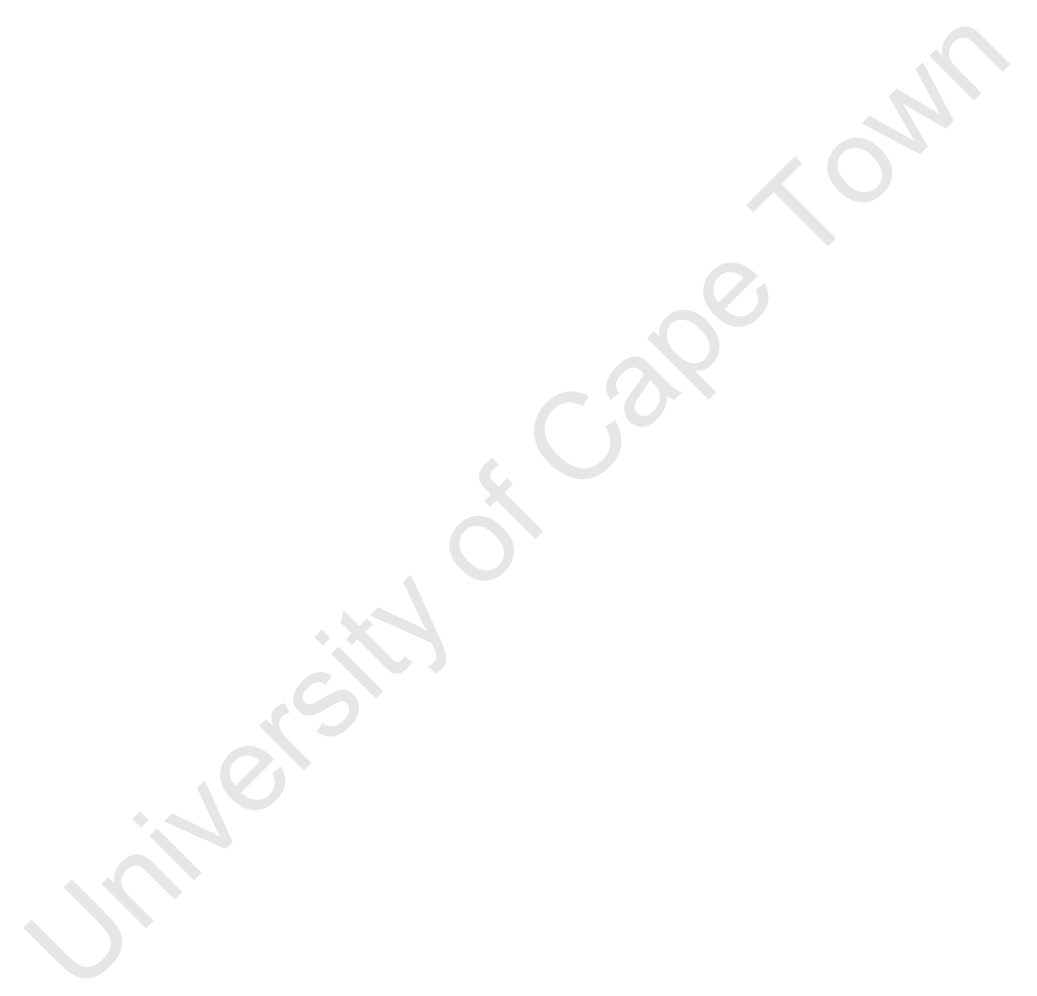




\section{Abstract}

Spark Ignition (SI) engine fuels' anti-knock properties are measured in the Co-operative Fuel Research (CFR) engine under two different test conditions as prescribed by the American Society for Testing and Materials (ASTM) for Research Octane Number (RON) and Motor Octane Number (MON) ratings. Recent research has been focused on determining whether the numerical difference between RON and MON, known as Octane Sensitivity (OS), is a result of the chemical or physical properties of the fuel. Reheating of the air-fuel mixture during the MON test aims to achieve full evaporation of the liquid fuel prior to entry into the intake port and cylinder. Under RON conditions, the air-fuel mixture is not reheated, and there is the possibility of fuel evaporative cooling to affect the final gas temperatures. Furthermore, the possibility of in-cylinder fuel evaporation could result in non-uniform temperature distributions within the cylinder.

The present research examined the effect that the operating environment has on fuel evaporation, and thus OS, in the CFR engine. A 3-dimensional (3D) Computational Fluid Dynamic (CFD) model of the CFR engine which accounts for the intake and compression cycle was developed. The carburettor function was approximated as a fuel spray and was modelled using a discrete particle method approach. The in-cylinder temperature distributions and heat transfer from the port and cylinder walls were studied for two fuels, iso-octane and toluene, under both RON and MON conditions.

It was found that, although a significant amount of liquid fuel entered the intake port and cylinder under RON conditions, the majority of the latent heat of vaporisation (LHV) was sourced from the hot cylinder walls, rather than resulting in a lasting evaporative cooling effect on the charge gasses. At the start of compression, radial and strong axial temperature gradients were set up in the cylinder under RON and MON conditions, with the radial profiles under RON conditions exhibiting asymmetry. The asymmetry of the radial temperature profiles and the strong axial temperature gradients observed were not obvious and not assumed in literature. The compression heating behaviour of the air-fuel mixture constituents - i.e. the value of the ratio of specific heats, known as gamma $(\gamma)$ - was shown to significantly influence 
final temperatures compared to evaporative cooling LHV effects on temperature development during intake. Even though the toluene-air-mixture benefited from a higher LHV for toluene compared to iso-octane, it was hotter at the end of compression. The combustion chamber wall temperature was also shown to play an important role in the temperature distribution within the cylinder. When the wall temperature was greater than the gas temperature prior to compression, it would heat the gas adjacent to it, resulting in hotter gas regions closer to the walls. For knock prediction in SI engines, these insights had implications when determining whether auto-ignition would occur, and also the location where it would initiate. 


\title{
Acknowledgements
}

\author{
Dr. Gareth Floweday and Prof. Chris Meyer
}

Thanks for the supervision, guidance and patience. Your time and effort are greatly appreciated.

\section{Sasol Advanced Fuels Laboratory}

Thanks for taking me on as a postgraduate student and providing adequate financial support. Thanks to the SAFL staff, in particular to Mr. Marlan Perumal and Prof. Andy Yates, for the interest and valuable input along the way.

\section{Prof. Daya Reddy and CERECAM}

Thanks for providing me with a lovely mountain view from my desk in CERECAM. Thanks also for providing access to a computer and a computing cluster to run my simulations on.

My friends: Kevin, JP, Bev, Jonathan, Kat, James, Dayne, Reuben, Richard, Tiri and Justin

Thanks for being around to discuss various topics. Thanks for all the language, IT, FLUENT $^{T M}$, GAMBIT $^{T M}$ and $\mathrm{AT}_{\mathrm{E}} \mathrm{X}$ support.

\section{Department of Mechanical Engineering}

Thanks to the staff at the UCT Department of Mechanical Engineering, in particular Prof. Genevieve Langdon, Prof. Gerald Nurick and Prof. Chris Redelinghuys, for the support. 


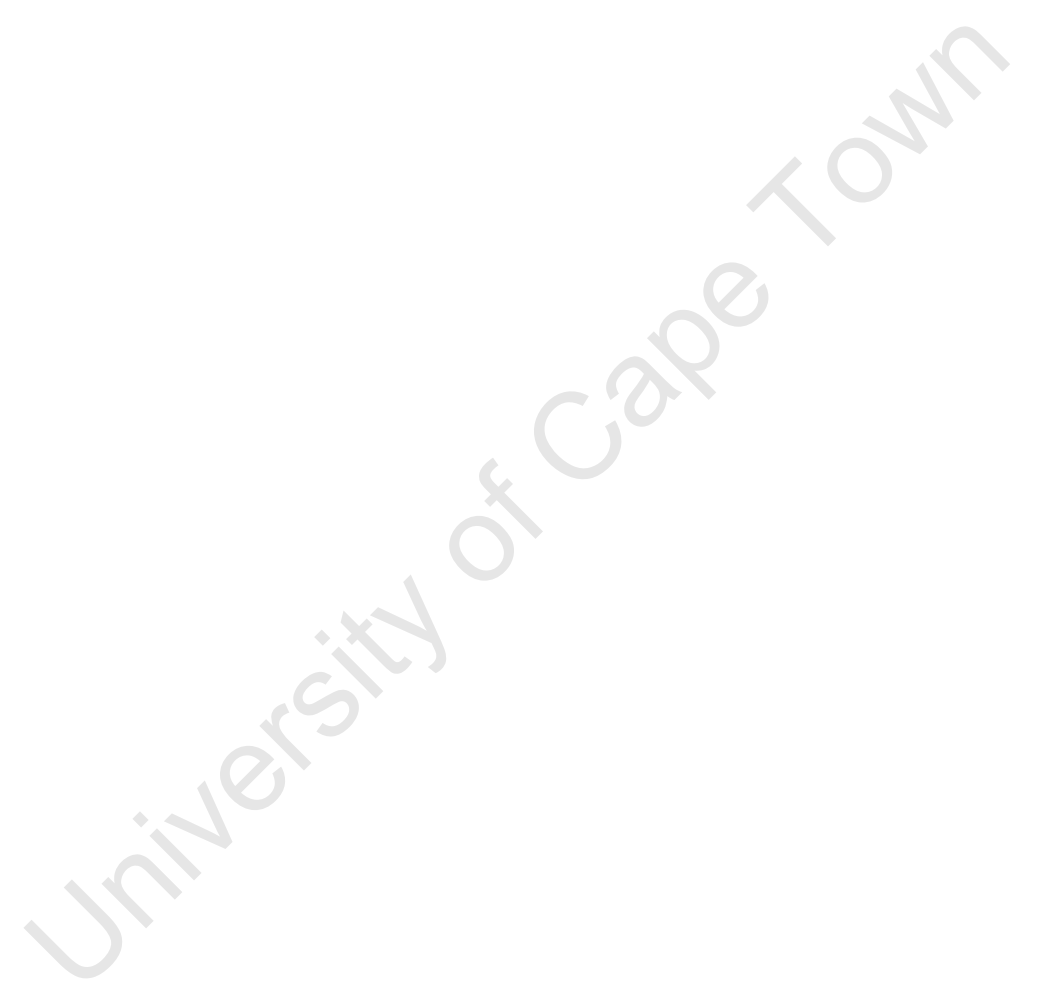




\section{Declaration}

1. I know that plagiarism is wrong. Plagiarism is to use another's work and pretend that it is one's own.

2. I have used the standard IEEE convention for citation and referencing. Each significant contribution to, and quotation in, this report from the work(s) of other people has been attributed, and has been cited and referenced.

3. This report is my own work.

4. I have not allowed, and will not allow anyone to copy my work with the intention of passing it off as his or her own work.

SIGNED:

Anja Thiart

THRANJ001 


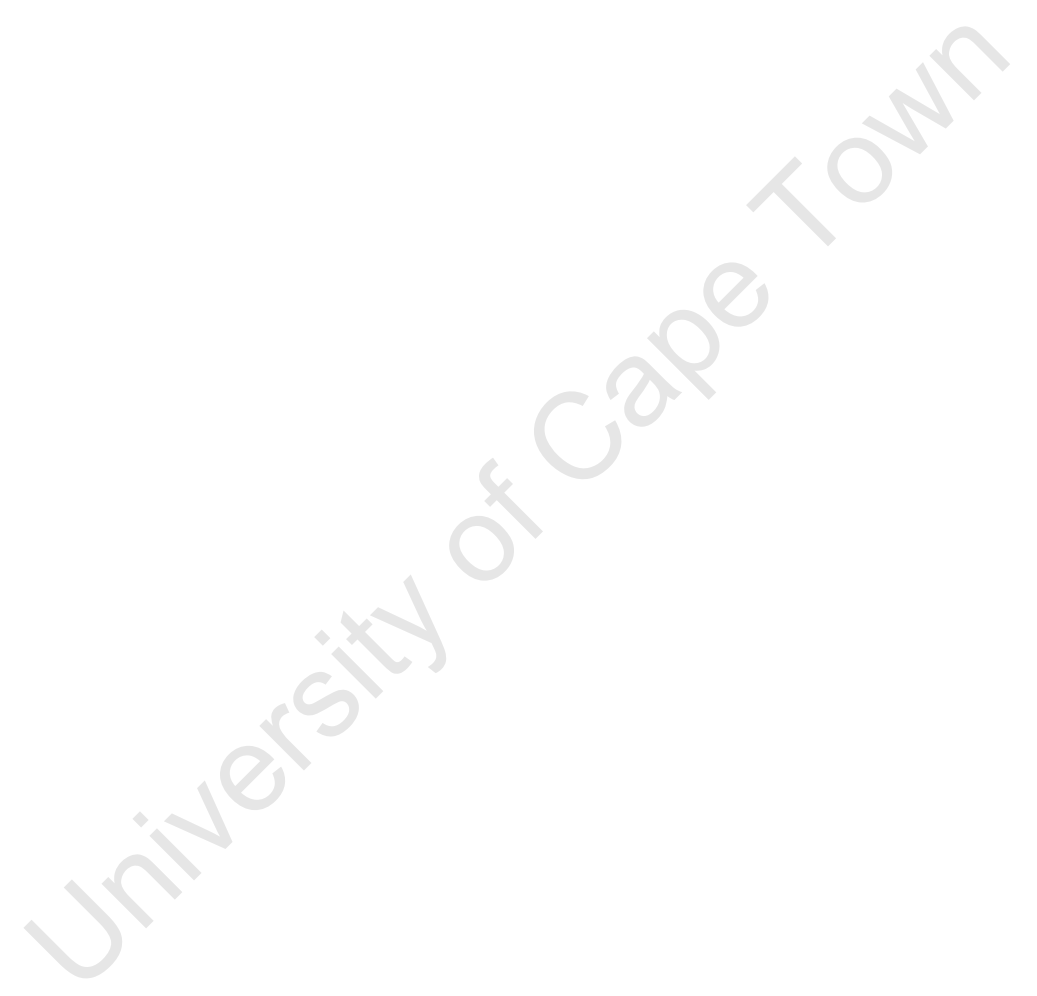




\section{Contents}

Abstract ii

Acknowledgements iii

Declaration v v

Contents ix

List of figures $\quad$ xii

List of tables $\quad$ xiii

Nomenclature $\quad$ xv

1 Introduction $1-1$

1.1 Combustion and knock in SI engines . . . . . . . . . . . . . . . . 1-1

1.2 Fuel anti-knock performance and Octane Sensitivity . . . . . . . . . . . . . 1-2

1.3 Current importance of octane numbers . . . . . . . . . . . . . . . . . . . . 1-3

1.4 Current research overview $\ldots \ldots \ldots \ldots$. . . . . . . . . . . . . 1-4

1.4 .1 Research objectives . . . . . . . . . . . . . . . . . . . . . . . 1-4

1.4 .2 Scope and limitations . . . . . . . . . . . . . . . . 1-5

1.4.3 Research approach . . . . . . . . . . . . . . . . . . . 1-5

1.4 .4 Thesis outline . . . . . . . . . . . . . . . . . 1-5

2 Literature review $2-1$

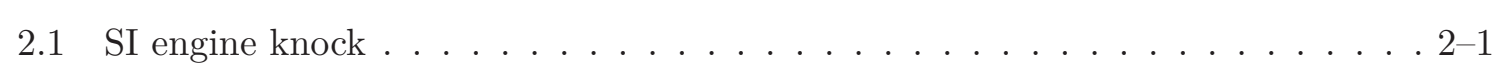

2.1 Auto-ignition of hydrocarbon fuels . . . . . . . . . . . . . . 2-2

2.2 Octane rating in the CFR engine . . . . . . . . . . . . . . . . 2-4

2.2 .1 Octane Sensitivity . . . . . . . . . . . . . . . . . 2-7

2.3 Engine modelling . . . . . . . . . . . . . . . . . . . . . . . . . 2-8

2.4 Liquid fuel spravs in SI engines . . . . . . . . . . . . . . . . . . . 2-11 
2.4 .1 Heat transfer . . . . . . . . . . . . . . . . . . . . 2-11

2.4 .2 Mass transfer . . . . . . . . . . . . . . . . . . . . . . . . . 2-13

2.4.3 Thermo-physical properties required for sprav evaporation modelling . . 2-14

2.5 Current questions surrounding OS, knock and evaporative cooling . . . . . . . 2-16

2.6 Summary . . . . . . . . . . . . . . . . . . . . 2-21

3 Computational Fluid Dynamics $3-1$

3.1 Numerical modelling of the continuous phase fluid flow . . . . . . . . . . . . . 3-1

3.1 .1 Pre-processing . . . . . . . . . . . . . . . . . . . . . . 3-1

3.1 .2 Governing equations . . . . . . . . . . . . . . . . . . . . 3-4

3.1.3 The Finite Volume Method for fluid flow . . . . . . . . . . . . . . . . . 3-5

3.1.4 Revnolds-averaged Navier-Stokes Equations . . . . . . . . . . . . . . . 3-6

3.1 .5 Turbulence modelling . . . . . . . . . . . . . . . . . . . . . . . . . 3-7

3.1.6 Discretization scheme and pressure-velocity coupling . . . . . . . . . . 3-9

3.1.7 Solution convergence: spacial and temporal discretization consideration 3-12

3.2 Numerical modelling of the discrete phase fluid flow . . . . . . . . . . . . . 3-12

3.2.1 Droplet particle trajectory and position . . . . . . . . . . . . . . . . 3-13

3.2.2 Heat transfer to and from the droplet particles . . . . . . . . . . . . . . 3-14

3.2 .3 Mass transfer to / from droplet particle . . . . . . . . . . . . . . . 3-14

3.2.4 Overall heat transfer to droplet . . . . . . . . . . . . . . . . . . . . . . 3-14

3.3 Numerical modelling of the wall film fluid flow . . . . . . . . . . . . . 3-15

3.4 Coupling between fluid and liquid phase . . . . . . . . . . . . . 3-16

4 Numerical approach $\quad$ 4-1

4.1 Initial models used in the development phase . . . . . . . . . . . . . . . . 4-1

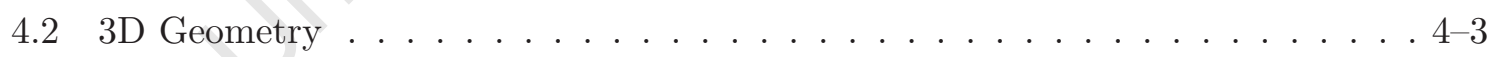

4.3 Computational grid . . . . . . . . . . . . . . . . . . . . . . . . . .

4.4 Dvnamic model setup . . . . . . . . . . . . . . . . . . 4-9

4.5 Solver setup . . . . . . . . . . . . . . . . . . . . . . . . . . . . 4-11

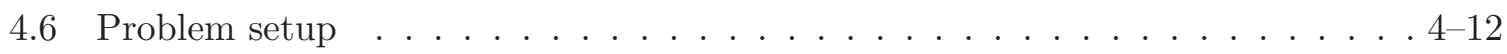

4.6 .1 Initial conditions . . . . . . . . . . . . . . . . . . . . . 4-13

4.6 .2 Boundary conditions . . . . . . . . . . . . . . . . . . . . 4-14

4.6 .3 Injection set-up . . . . . . . . . . . . . . . . . . . . . . . . 4-16

4.6 .4 Materials set-up . . . . . . . . . . . . . . . . . . 4-16

4.7 Modelling procedure sequence . . . . . . . . . . . . . . . . . . . 4-16

5 Results $5-1$

5.1 Model development . . . . . . . . . . . . . . . . . . . . . 5-1

5.1 .1 AFR UDF . . . . . . . . . . . . . . . . . . . . 
5.1.2 MON mixture temperature calibration using the 3D model . . . . . . . 5-6

5.2 Results of the final 3D models . . . . . . . . . . . . . . . . . . . . 5-11

5.2 .1 Evaporative cooling . . . . . . . . . . . . . . . . . . 5-11

5.2 .2 In-cvlinder temperature distribution . . . . . . . . . . . . . . . 5-16

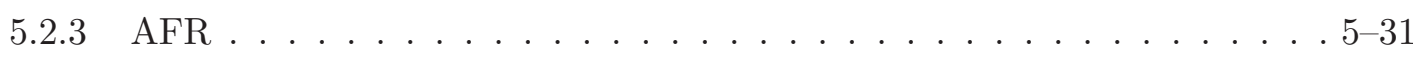

6 Discussion of results $\quad 6-1$

6.1 Evaporative cooling . . . . . . . . . . . . . . . . . . . . 6-1

6.2 In-cylinder temperature distribution . . . . . . . . . . . . . . 6-2

6.3 Combustion chamber wall temperatures . . . . . . . . . . . 6-6

6.4 Fixed temperature wall boundary conditions . . . . . . . . . . . 6-7

7 Conclusions $7-1$

8 Recommendations 8-1

8.1 Further CFD modelling with the existing model . . . . . . . . . . . . . . . . . 8-1

8.2 Simple 0D and 1D engine modelling to investigate $\gamma$ effects . . . . . . . . 8-1

8.3 Improvement of the results obtained from CFD analyses . . . . . . . . . . . 8-2

8.4 Experimental work required to provide better initial and boundary conditions . 8-2

8.5 Combustion and auto-ignition modelling using full 3D CFD . . . . . . . . . . 8-3

\begin{tabular}{|l|l} 
A User-defined functions & $\mathbf{A}-1$
\end{tabular}

A.1 DPM mass flow rate UDF . . . . . . . . . . . . . . . . A-1

$\begin{array}{ll}\text { References } & \text { Ref-1 }\end{array}$ 


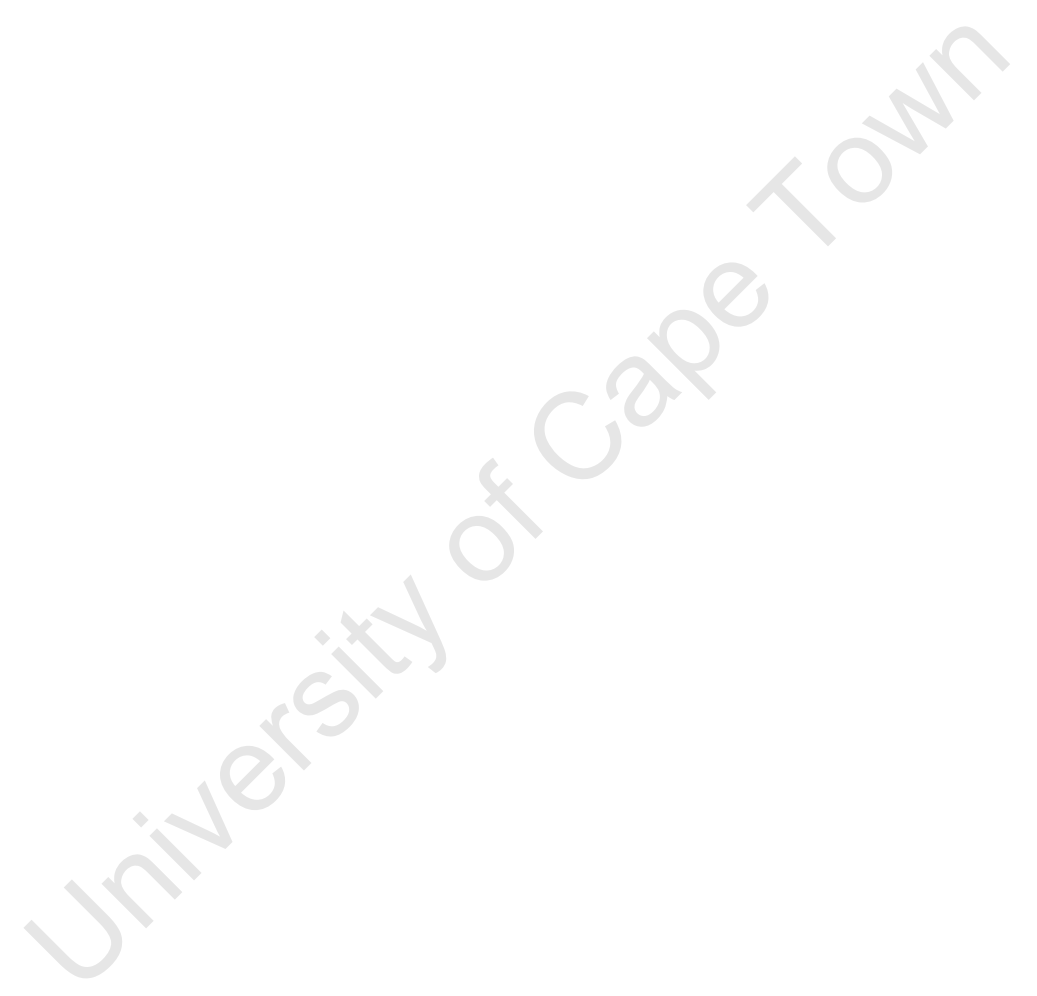




\section{List of Figures}

2.1 Normal and knocking combustion . . . . . . . . . . . . . . . . 2-1

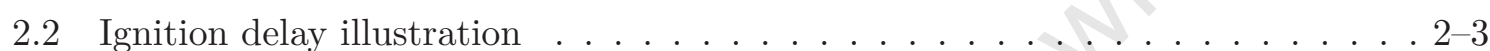

2.3 The CFR engine . . . . . . . . . . . . . . . . . . . . . . . . 2-5

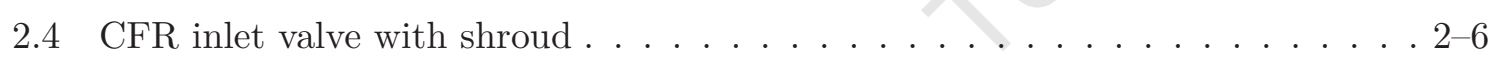

2.5 Engine schematid . . . . . . . . . . . . . . . . . . . .

3.1 Types of mesh elements . . . . . . . . . . . . . . . . . . . . . . . . . . . . . . . . . . .

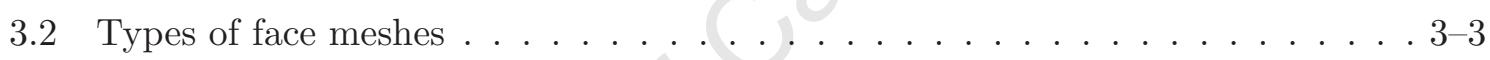

3.3 Skewness range for quadrilateral elements . . . . . . . . . . . . . . . 3-3

3.4 Boundary laver showing buffer zone . . . . . . . . . . . . . . . . . 3-9

3.5 Flow chart for SIMPLE algorithm . . . . . . . . . . . . . . . . 3-11

3.6 Wall film: physical processes . . . . . . . . . . . . . . . . 3-15

4.1 Geometrv and boundary conditions for the $2 \mathrm{D}$ manifold model . . . . . . . . . 4-1

4.2 Geometry used for the $2 \mathrm{D}$ planar model . . . . . . . . . . . . . . . . . 4-2

4.3 CFD model geometries used for 3D simulations . . . . . . . . . . . . . . . 4-4

4.4 Inlet port with shrouded inlet valve . . . . . . . . . . . . . . . . 4-5

4.5 Grid $\mathrm{MON} \ldots \ldots \ldots \ldots \ldots \ldots \ldots$

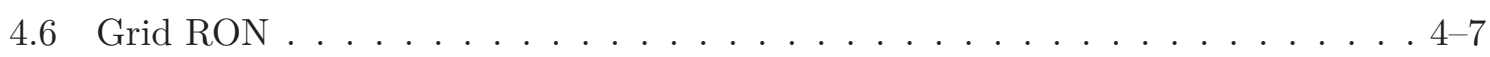

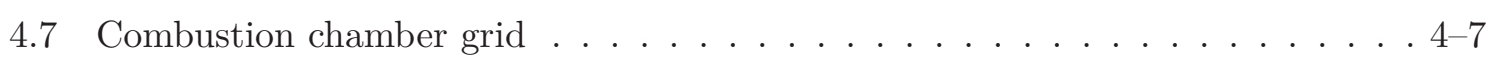

4.8 Dynamic mesh lavering above and below the inlet valve . . . . . . . . . . 4-8

4.9 General engine geometry and parameters . . . . . . . . . . . . . 4-9

4.10 Special considerations during problem setup (MON) . . . . . . . . . . . . . . . 4-12

4.11 Special considerations during problem setup (RON) . . . . . . . . . . . . . 4-13

$5.1 n$-Hexane liquid sprav: Average static temperature vs manifold position . . . . 5-2

5.2 Average static temperature vs. distance along manifold for iso-octane . . . . . 5-3

5.3 Average in-cylinder $\lambda$ vs. crank angle for RON and MON setup with iso-octand 5-5

5.4 MON heating manifold and mixture temperature measurement location . . . . 5-7

5.5 3D Heating $\mathrm{T}$ BC calibration with air . . . . . . . . . . . . . 5-8 


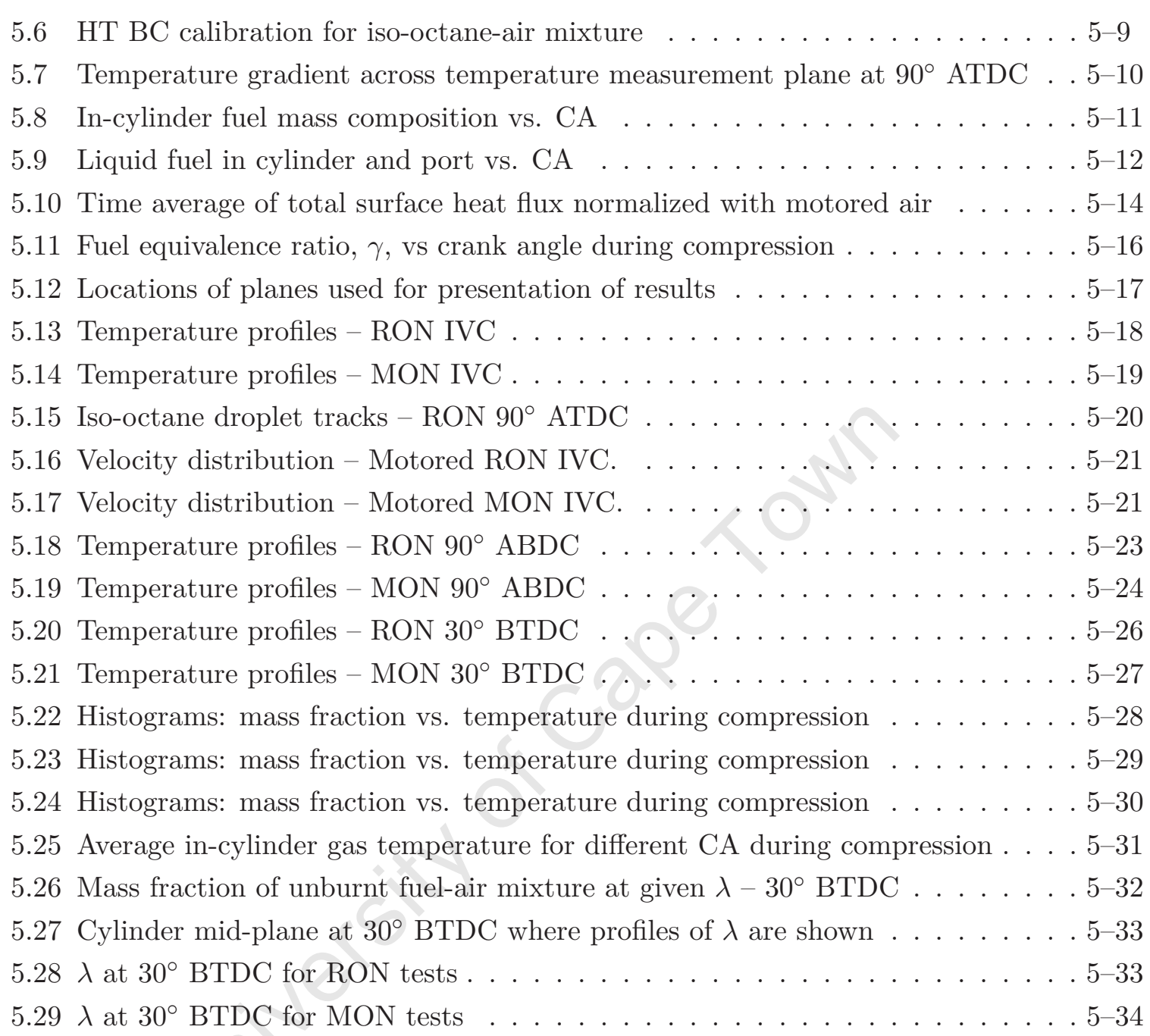

6.1 Assumed initial radial temperature profiles used in literature . . . . . . . . 6-3

6.2 Diagram showing lines along which temperature profiles are plotted . . . . . 6-3

6.3 Radial temperature profiles at IVC for MON tests . . . . . . . . . . . . . 6-4

6.4 Radial temperature profiles at IVC for MON tests . . . . . . . . . . . 6-5

6.5 Radial temperature profile associated with higher chamber wall temperatures. . 6-6 


\section{List of Tables}

2.1 CFR engine parameters . . . . . . . . . . . . . . . 2-6

2.2 CFR engine operating conditions . . . . . . . . . . . . . . . . . 2-7

2.3 Thermophysical properties . . . . . . . . . . . . . . . . . . . . . . 2-14

4.1 Engine parameters for in-cylinder model . . . . . . . . . . . . . . . . . . 4-10

4.2 In-cvlinder conditions after the exhaust stroke at IVO . . . . . . . . . . . . . . 4-13

4.3 BCs at the air inlet . . . . . . . . . . . . . . . . . . . . . 4-14

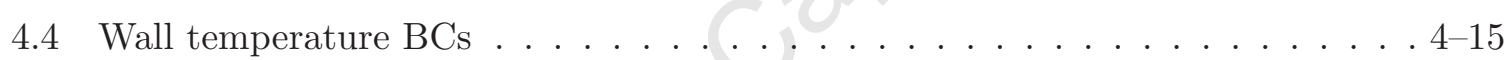

4.5 Summary of injection properties $\ldots \ldots \ldots \ldots$. . . . . . . . . . . . . .

5.1 Final HT BC chosen for heating elements . . . . . . . . . . . . . . . . . . . 5-10

5.2 Cooling potential of the liquid fuel in port and cylinder . . . . . . . . . . . 5-13

5.3 Average $\lambda$ at $30^{\circ}$ BTDd . . . . . . . . . . . . . . . . . . . . . . . . . . . 


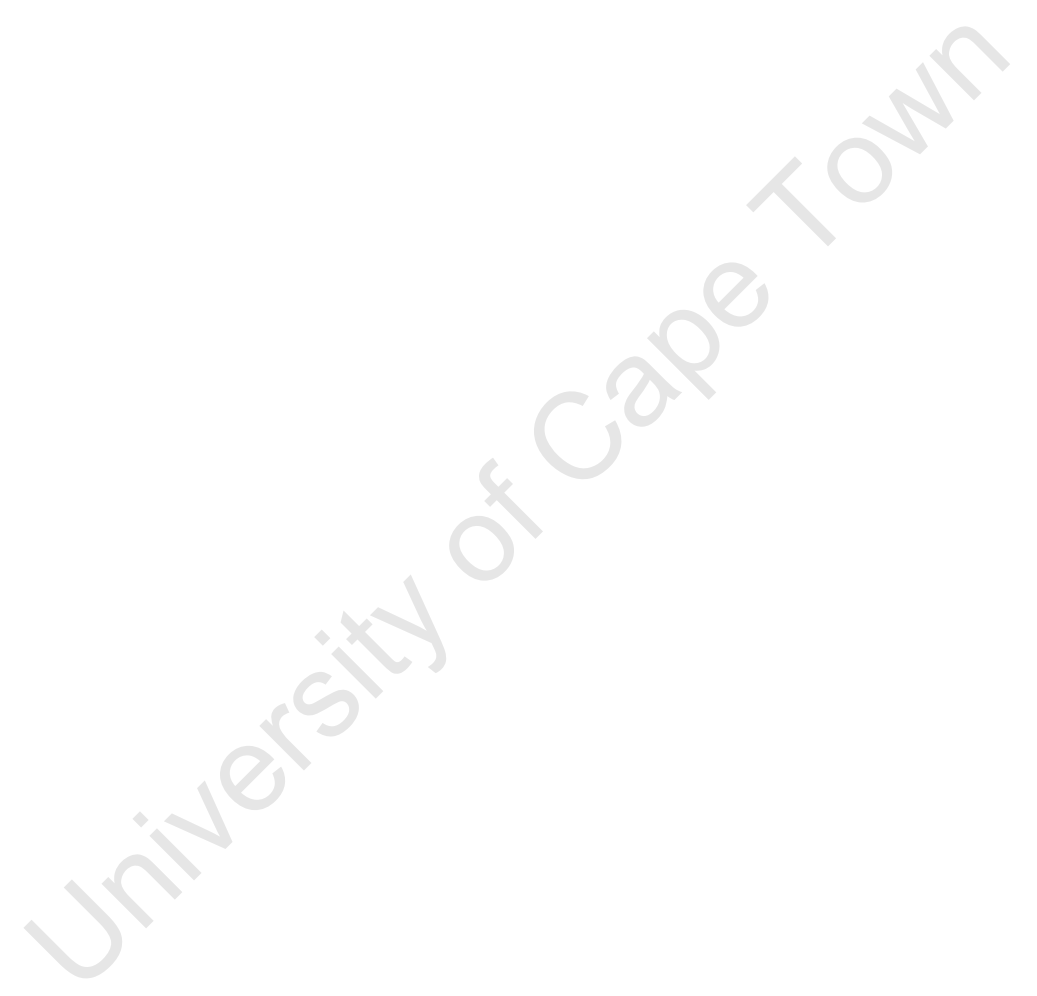




\section{Nomenclature}

\section{Symbols}

$\boldsymbol{u}$

A

a

C

c

D

$d$

E

F

$F^{\prime}$

$g$

$h$

$i$

$k$

$k$

$k$

$L$

l

m

$r$

$s$

$t$

V

$v_{r}$

$x_{p}$

$\mathrm{Nu}$

$\mathrm{P}$

$\operatorname{Pr}$
Velocity vector $\left(m . s^{-1}\right)$

Area $\left(m^{2}\right)$

Crank radius $(\mathrm{mm})$

Concentration $\left(\mathrm{kg} \cdot \mathrm{m}^{-3}\right)$

Specific heat capacity $\left(J \cdot \mathrm{kg}^{-1} \cdot \mathrm{K}^{-1}\right)$

Diffusion coefficient $\left(m^{2} \cdot s^{-1}\right)$

Diameter $(m)$

Energy $(J)$

Force $(N)$

Force per unit particle mass

Gravitational acceleration $\left(m . s^{-2}\right)$

Heat transfer coefficient $\left(W \cdot m^{-2} \cdot K^{-1}\right)$

Specific internal energy $\left(J . \mathrm{kg}^{-1}\right)$

Mass transfer coefficient $\left(m . s^{-1}\right)$

Thermal conductivity $\left(W \cdot m^{-1} \cdot K^{-1}\right)$

Turbulent kinetic energy $(J)$

Length $(m)$

Connecting rod length $(\mathrm{mm})$

Mass $(k g)$

Radius $(m)$

Stroke $(\mathrm{mm})$

Time $(s)$

Volume $\left(m^{-3}\right)$

Relative velocity $\left(m . s^{-1}\right)$

Piston displacement measured from TDC $(\mathrm{mm})$

Nusselt number

Pressure $(\mathrm{Pa})$

Prandtl number 
$\mathrm{R}$

Re

Sh

$\mathrm{T}$

\section{Greek Letters}

$\epsilon$

$\gamma$

$\lambda$

$\mu$

$\mu_{t}$

$\bar{\tau}_{w}$

$\tau$

\section{Subscripts}

$\infty$

d

g

1

$\mathrm{w}$

Specific gas constant $\left(k J . k g^{-1} \cdot K^{-1}\right)$

Reynolds number

Sherwood number

Temperature $(K)$

Dissipation rate of $k\left(m^{2} \cdot s^{-3}\right)$

Gamma

Mixture equivalence ratio

Dynamic viscosity $\left(k g \cdot m^{-1} \cdot s^{-1}\right)$

Eddy viscosity $\left(\mathrm{kg} \cdot \mathrm{m}^{-1} \cdot \mathrm{s}^{-1}\right)$

Average wall shear stress $(P a)$

Induction time $(m s)$

In the bulk gas

Droplet

Gas phase

Liquid phase

Wall

\section{Abbreviations}

$L H V$

ABDC

Latent heat of vaporisation $\left(J . \mathrm{kg}^{-1}\right)$

AFR

After BDC

ASTM

Air to Fuel mass Ratio

ATDC

BDC

American Society for Testing and Materials

After TDC

CARS

Bottom Dead Centre

CFD

Coherent Anti-Stokes Raman Spectroscopy

CFR

Computational Fluid Dynamics

CR

Co-operative Fuel Research

$\mathrm{CV}$

Compression Ratio

DPM

Control Volume

FVM

Discrete Particle Method

HCCI

Finite Volume Method

IC

Homogeneous Charge Compression Ignition

IVC

Internal Combustion

Inlet Valve Close 


$\begin{array}{ll}\text { IVO } & \text { Inlet Valve Open } \\ \text { LHV } & \text { Latent Heat of Vaporisation } \\ \text { MON } & \text { Motor Octane Number } \\ \text { MUSCL } & \text { Monotone Upstream-centred Scheme for Conservation Laws } \\ \text { ON } & \text { Octane Number } \\ \text { OS } & \text { Octane Sensitivity } \\ \text { PDE } & \text { Partial Differential Equation } \\ \text { PDPA } & \text { Phase Doppler Particle Anemometry } \\ \text { PISO } & \text { Pressure Implicit with Splitting of Operators } \\ \text { PIV } & \text { Particle Image Velocimetry } \\ \text { PLIF } & \text { Planar Laser-induced Fluorescence } \\ \text { PRF } & \text { Primary Reference Fuel } \\ \text { QUICK } & \text { Quadratic Upstream Interpolation for Convective Kinetics } \\ \text { RANS } & \text { Reynolds Averaged Navier-Stokes } \\ \text { REG } & \text { Residual Exhaust Gas } \\ \text { RON } & \text { Research Octane Number } \\ \text { RPM } & \text { Revolutions Per Minute } \\ \text { SAFL } & \text { Sasol Advanced Fuels Laboratory } \\ \text { SI } & \text { Spark Ignition } \\ \text { SIMPLE } & \text { Semi-implicit Pressure Linked Equations } \\ \text { SIT } & \text { Spark Ignition Timing } \\ \text { TDC } & \text { Top Dead Centre } \\ \text { TDMA } & \text { Tri-diagonal Matrix Algorithm } \\ \text { UCT } & \text { University of Cape Town } \\ \text { UDF } & \text { User-defined Function }\end{array}$




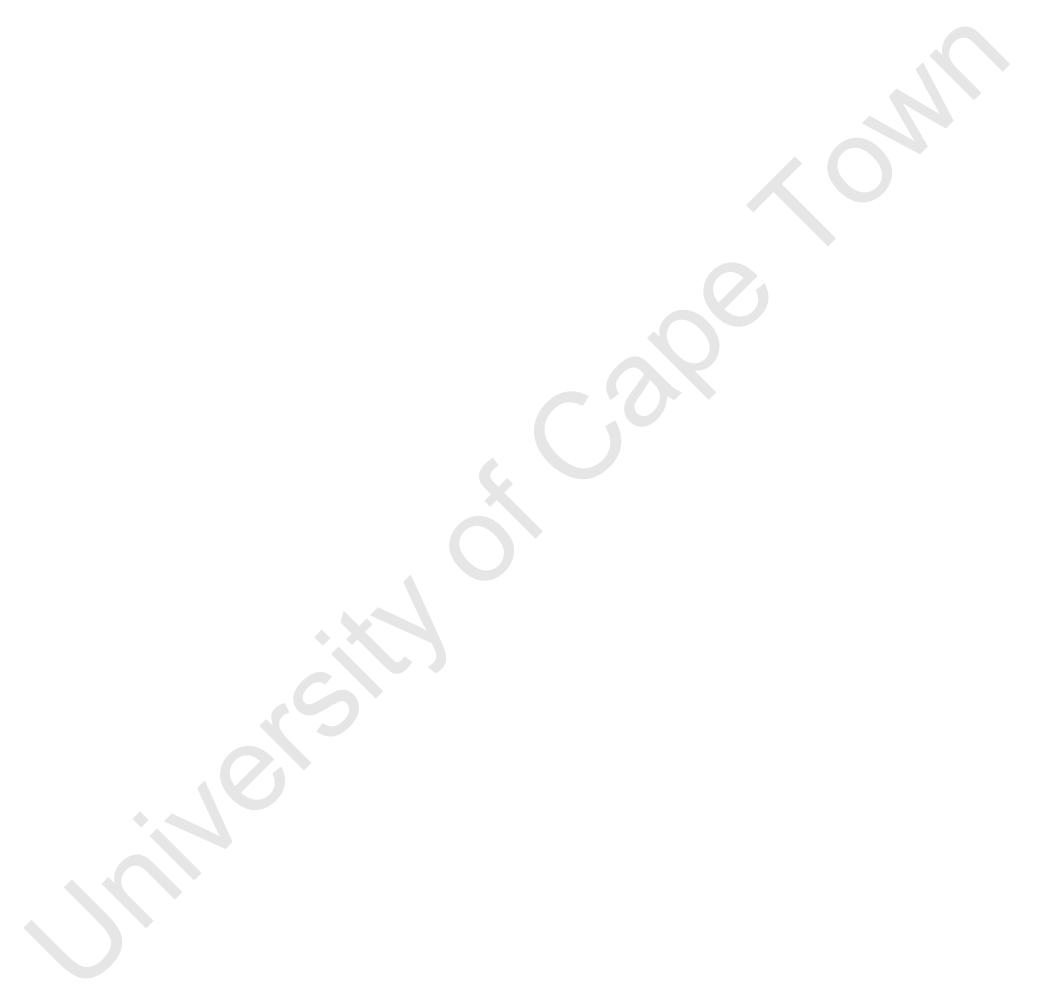




\section{Introduction}

This chapter gives a brief overview of the project topic, the reason for the project and the current research objectives and scope, as well as a short orientation of the thesis layout.

\subsection{Combustion and knock in SI engines}

Octane Number (ON) is a fuel property which relates to combustion in Spark Ignited (SI) engines. Combustion in SI engines can be divided into normal and abnormal combustion, where normal combustion is desired for optimal operation. During normal combustion the fuel-air mixture is ignited by a spark which provides the necessary energy for the chemical combustion reactions to initiate. The fuel-air mixture then burns out steadily from the sparkplug in a progressive turbulent flame propagation. Conversely, knocking combustion relates to an unwanted combustion event in SI engines known as auto-ignition. Auto-ignition occurs where some or all of the unburnt gas 1 ignites spontaneously without some external ignition source before the advancing flame front is able to consume the unburnt gas. In SI engines, the term 'knock' is used to describe the engine noise caused by this rapid auto-ignition of unburnt gas [1].

In order to increase the thermal efficiency of an SI engine, the compression ratio (CR) must be increased. However, increasing the CR also increases the unburnt gas pressure and temperature. At higher temperatures, the engine is more prone to knock. During knocking combustion, the auto-ignition of the unburnt gas creates localized pressure spikes and gas turbulence, which can be harmful to the engine due to the associated increase in the combustion chamber heat transfer. Engine knock can thereby cause damage to expensive engine components [1, 2].

The onset of knock in SI engines effectively limits the CR and thus the overall engine efficiency. The design of engines and fuels to reduce knock tendency while pushing the CR to the limit

\footnotetext{
${ }^{1}$ The unburnt gas refers to the air-fuel mixture present prior to ignition as well as the unburnt air-fuel mixture ahead of the flame after ignition.
} 
is therefore fundamental to engine fuel development.

\subsection{Fuel anti-knock performance and Octane Sensitivity}

Engine design and operating variables influence a fuel's tendency to knock because of the associated unburnt gas conditions such as temperature and pressure. However, the presence, or absence, of knock in engines depends primarily on the anti-knock quality of the fuel [1]. In order to compare different fuel blends, a practical measure of a fuel's resistance to knock is used. This measure is defined by the octane scale and measured according to standard octane rating methods [3, 4] performed using the Cooperative Fuels Research (CFR) engine [5].

The ON scale is based on the resistance to knock of the two hydrocarbons $n$-heptan 2 and iso-octan 3 , which are defined as having ONs of 0 and 100 respectively. These hydrocarbons differ greatly in their ability to resist knock, with iso-octane having the higher resistance to knock. A blend of $90 \%$ iso-octane and $10 \% n$-heptane has an ON of 90 . A fuel's ON is determined by measuring what blend of iso-octane and $n$-heptane matches the fuel's knock resistance 4 . The fuel sample is in turn rated using two different procedures, the research and motor methods, which result in two different ON, namely the Research Octane Number (RON) and the Motor Octane Number (MON) [1, 5].

These two procedures were originally intended to represent the typical on-road operation of a vehicle. The RON method [3] correlated with full-scale spark-ignited engine anti-knock performance at low speed. The MON method [4] represented more severe engine operating conditions which are more likely to produce knock [1]. The numerical difference between these two ON values is known as the fuel's Octane Sensitivity (OS) defined as:

$$
\text { Octane Sensitivity }=\text { RON - MON }
$$

The main difference between the two tests methods is the way in which the inlet fuel-air mixture temperature is specified. During the MON test, the air-fuel mixture is reheated to $149^{\circ} \mathrm{C}$ in the inlet manifold and hence it is assumed that most of the fuel is evaporated before it enters the cylinder [6]. The RON test does not provide for any re-heating of the mixture, and only the inlet air temperature is specified at $52^{\circ} \mathrm{C}$. There is thus a much greater possibility of fuel evaporation during the inlet and compression stroke to affect the in-cylinder temperature and its distribution at the time of ignition. The other main difference is the engine speed $600 \mathrm{rpm}$ for RON and $900 \mathrm{rpm}$ for MON. The RON tests thus results in a $50 \%$ reduction of

\footnotetext{
${ }^{2} \mathrm{n}$-Heptane is a straight chain paraffin

${ }^{3}$ Iso-octane is also known as 2,2,4-Trimethylpentane, and is a branched chain paraffin

${ }^{4}$ This is discussed more extensively in subsequent chapters
} 
auto-ignition development times w.r.t. that of the MON test.

Opinions differ widely when it comes to an explanation for high values of OS. Some studies [7, 8] attribute OS entirely to evaporative cooling effects associated with different classes of fuels (e.g. paraffins, olefins, aromatics and alcohols). Other studies ascribe the difference to a chemical distinction [9, 10. This conflict arises from the huge difficulty in determining the exact value of the gas temperature profile in the engine that is set up to perform the research method. Studies have been done on the measurement of the in-cylinder temperature in the CFR engine [11, 12], but difficulty lies in knowing what temperature is being measured gas, liquid or a combination. Additionally, the spatial variation of temperature is of interest, making direct measurements more complicated.

\subsection{Current importance of octane numbers}

Today, gasoline specifications in South Africa specify a minimum of 91/93/95 RON and 85 MON5. About a third of the country's fuel is synthetically produced in a coal-based FischerTropsch plant operated by Sasol [13]. The onset of the phasing out of octane enhancing heavy metal fuel additives (such as lead in 2006) has placed emphasis on developing fuels with intrinsically higher octane.

The refinery processes have to be designed to produce fuel compositions with higher octane ratings. Low-octane components need to be separated out, while high-octane components need to be produced or synthesized. The high energy input and operational costs associated with these processes are joined by capital investment costs to render octane one of the most expensive properties of fuel today. It is thus important to understand octane measurement at a fundamental level.

The Sasol advanced fuels laboratory (SAFL) has thus returned to the problem of octane measurement and the nature of auto-ignition during the octane rating of gasoline fuels [13]. In particular, attention has been given to the influence of both the chemical and physical properties of the fuel. Since the octane scale is calibrated against a blend of primary reference fuels with defined octane numbers, the evaporative characteristics of these reference fuels are entrenched in the octane scale [6].

\footnotetext{
${ }^{5}$ These values differ slightly depending on altitude.
} 


\subsection{Current research overview}

In order to understand better the discrepancy between the RON and MON measurements, or rather OS, this study was focused on the engine operating environment and its contribution to fuel performance. In particular, the effect of fuel evaporation due to the physical environment was examined. The current research was not intended to prove that OS is due mainly to either chemical or physical attributes. Rather, the aim was to study the physical processes that take place during intake and compression in order to gain a better understanding of OS in the CFR engine.

The current research forms part of an ongoing effort at SAFL to be able to predict the ON of a fuel solely from its physical and chemical properties. Chemists can characterize most fuel properties from a very small sample of synthesized fuel (a few millilitres). However, an experimental octane determination requires several litres of fuel, which can become time consuming and expensive. Since ON plays such a huge role in understanding SI engine knock, it is important to fully understand the underlying physical processes involved during the octane rating of gasoline fuels.

\subsubsection{Research objectives}

For engine knock prediction, it is vital to know the magnitude and profile of the mixture temperature set up during octane rating and this project was intended to provide an improved estimate of this parameter. It therefore made sense to utilize computational methods to study the in-cylinder conditions set up during octane rating of fuels in the CFR engine.

The aims of the current research were thus to:

- Develop a CFD model of the intake and compression cycle of the CFR engine based on the octane rating tests.

- Determine the extent of liquid fuel present in the engine during intake and compression.

- Determine the extent of in-cylinder fuel evaporation.

- Determine the temperature profile set up in the cylinder prior to, and after, compression.

- Compare results for both the RON and MON cases for single component fuels with and without OS, as well as motored conditions.

- Compare results to other similar studies found in the literature. 


\subsubsection{Scope and limitations}

This project was not scoped to build a complete CFD model of the CFR engine. Only the intake stroke was studied. Where necessary, other cycles were approximated using boundary conditions. Operating conditions relating to the RON and MON tests were studied, with the single component fuel iso-octane 6 . Additionally toluene, a fuel which exhibits high OS, was also studied. No radiation heat transfer or chemical kinetics were included in the computational model.

\subsubsection{Research approach}

The following approach was used:

1. A 2D geometry of the CFR engine's intake and compression system was created. This included the inlet manifold, inlet port and valve, and the cylinder.

2. This geometry was extended so that it included the dynamic motion of the valve and piston.

3. The dynamic model was then developed fully to include the necessary physical complexity such as gas turbulence, species transport, liquid fuel evaporation models and wall film flow. This also entailed implementing a user-defined function to govern the liquid fuel spray flow rate.

4. Once fully developed, the 2D model was extended to 3D.

5. Studies were then conducted for toluene and iso-octane under both RON and MON conditions.

6. Results of these were then compared to motored cases with air under RON and MON conditions respectively in order to compare the heat transfer and temperature profiles which result when fuel is used.

\subsubsection{Thesis outline}

The thesis begins with an overview of the relevant literature. Previous work done, as well as the physics and tools required to understand the work are discussed. The next chapter explains in depth the approach taken in developing and setting up the CFD model. This is then followed by the results and discussions. Finally conclusions are drawn based on these discussions followed by any relevant recommendations.

\footnotetext{
${ }^{6} n$-Heptane and $n$-hexane were also used in initial studies.
} 


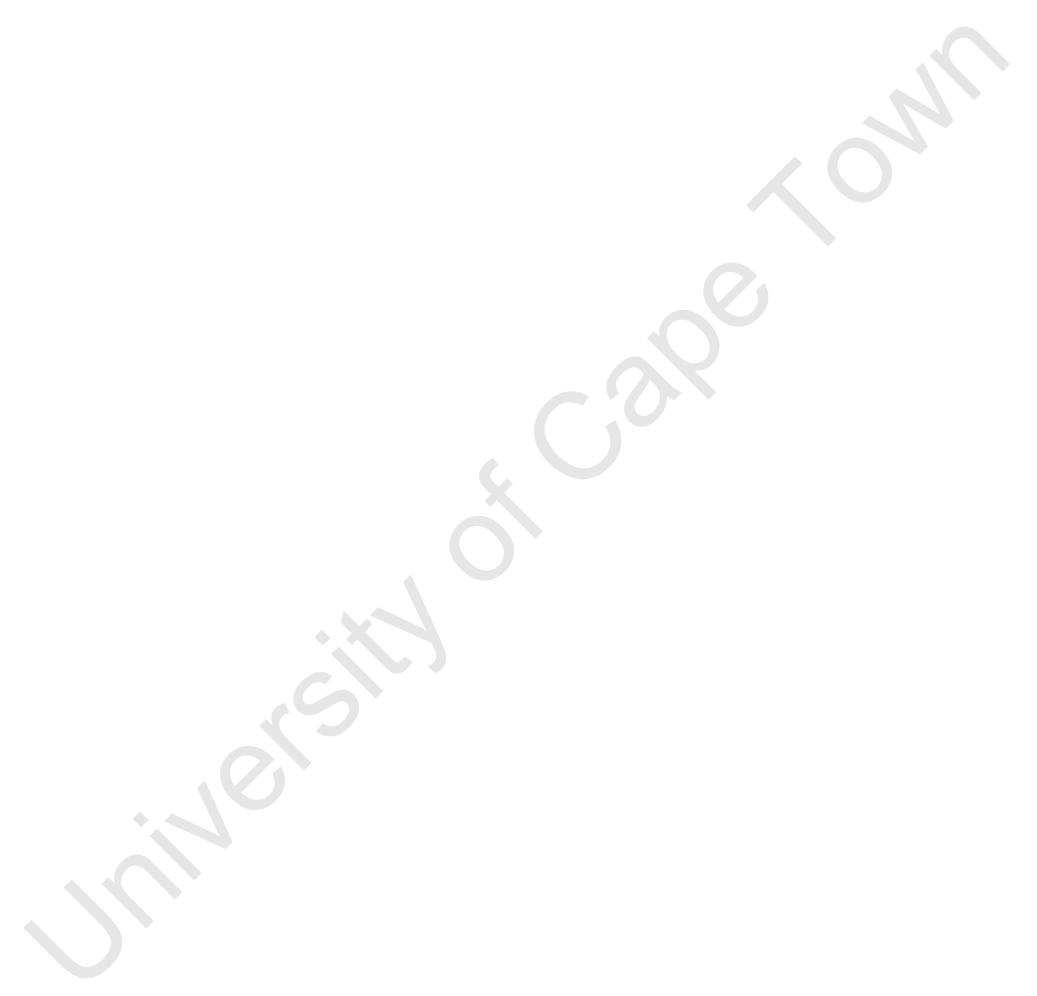




\section{Literature review}

This chapter starts by explaining knock and auto-ignition in SI engines and the CFR engine in particular. Although the modelling of these phenomena was outside the scope of this research, it formed the basis for the work that was done; the current work was necessary in order to gain more insight in the field of engine knock. Next, the background to and work done in octane rating is discussed, with the emphasis on OS. The physical processes involved in the current work are then covered and, finally, the implementation into a computational framework is discussed.

\subsection{SI engine knock}

Engine knock was briefly described in the introduction. This section provides more insight into this phenomenon and the underlying physics and chemistry relating to knock.

Figure 2.1 shows the in-cylinder pressure time records for normal to intense knocking combustion.

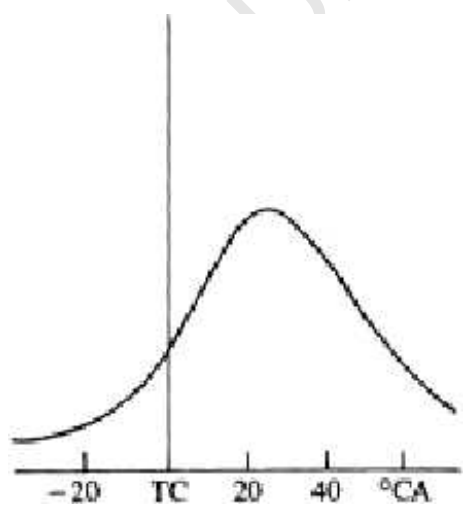

(a) Normal combustion, spark $28^{\circ} B T D C$

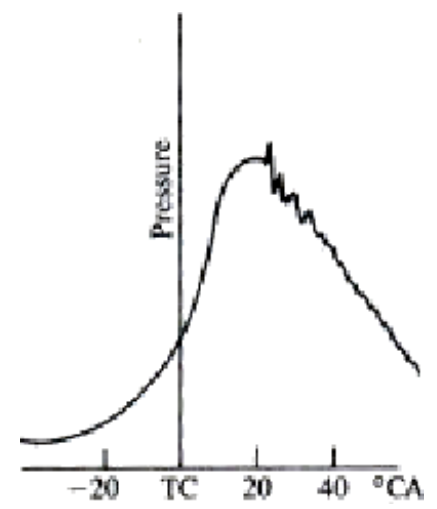

(b) Slight knock, spark $28^{\circ} B T D C$.

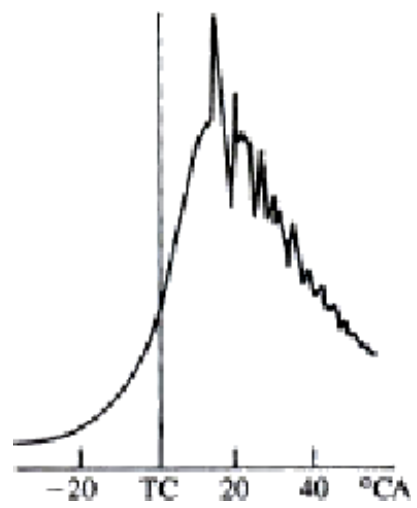

(c) Intense knock, spark $32^{\circ} B T D C$.

Figure 2.1: Cylinder pressure vs. crank angle traces for a $4000 \mathrm{rpm}$, single-cylinder engine at wideopen throttle [1]. 
The high frequency pressure fluctuations visible during knocking combustion are caused by the spontaneous local increase in gas pressure and temperature, thereby causing a shockwave to propagate away from the unburnt gas region across the combustion chamber. These waves are then reflected by the chamber walls and create the oscillatory pressure vs. time records exhibited in Figure 2.1. The larger the amplitudes of these fluctuations, the more intense the knocking [1]. Fundamental theories of knock are based on models for the auto-ignition of the fuel-air mixture in the unburnt gas [11].

\subsubsection{Auto-ignition of hydrocarbon fuels}

When hydrocarbons are oxidized they can behave in one or more of the following ways, depending on the temperature of the mixture [1]:

1. Slow reactions which occur at low pressures and temperatures below $470 \mathrm{~K}$. These types of phenomena do not typically occur in engines.

2. Single or multiple cool flames. At $570 \mathrm{~K}$ to $670 \mathrm{~K}$ small fractions of the gas react causing a slight temperature rise of only tens of degrees. These are called cool flames.

3. Two-stage ignition. Depending on the temperature and fuel, the above mentioned cool flame may be followed by a 'hot flame' or high temperature explosion where the reaction accelerates rapidly after ignition.

4. Single-stage ignition (hot flame) occurs where the fuel does not exhibit the cool flame or two-stage ignition behaviour. After the initial induction period the reaction takes place and accelerates rapidly.

The above is illustrated in Figure 2.2 for a sample fuel-air mixture by showing the temperature history for ignition of the sample fuel over a range of initial gas temperatures. 


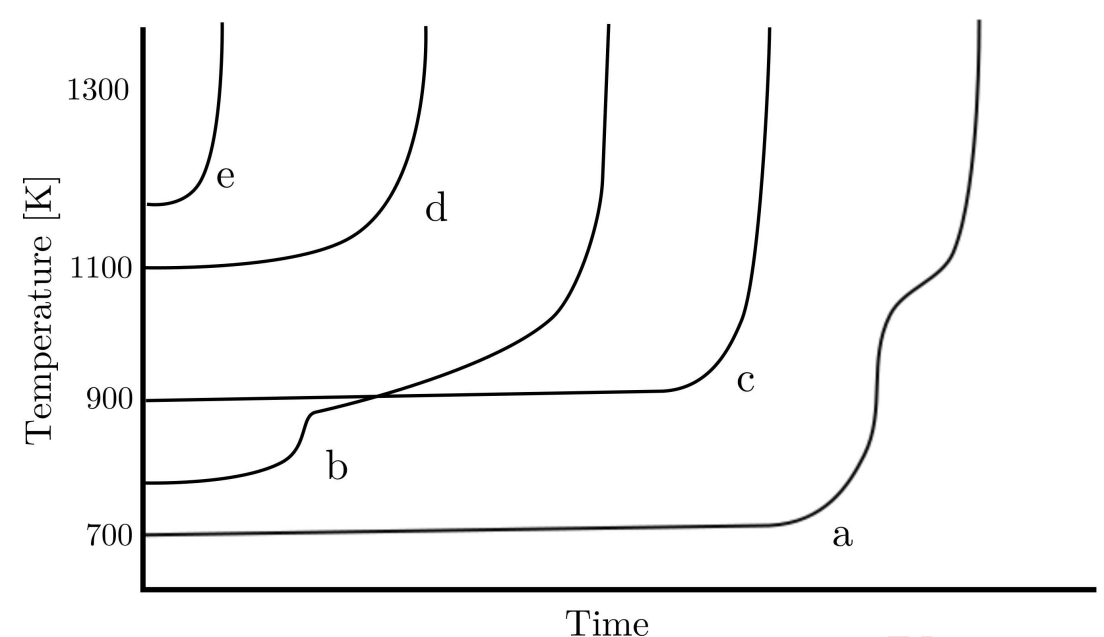

Figure 2.2: Temperature-time records for ignition of a sample fuel-air mixture over a range of initial gas temperatures - adapted from [11].

For different initial temperatures the ignition behaviour is as follows:

c, d, e - single-stage ignition with no cool flame

b - two-stage ignition with cool flame

a - two-stage ignition with initial cool flame heat release

For this sample fuel, at a particular pressure and air-fuel mass ratio (AFR), two-stage ignition is observed below initial temperatures of $900 \mathrm{~K}$, while single-stage ignition with no cool flames occurs above $900 \mathrm{~K}$.

This serves to show that auto-ignition is highly sensitive to temperature. Certain fuels may behave in a two-stage manner below a given initial temperature, whereas others may do so below much higher initial temperature conditions. Below a certain temperature a fuel which exhibits two-stage behaviour would not knock, however if this temperature changes slightly, the fuel could behave in a single-stage manner and knock. Normal paraffins give strong cool flames (two-stage), while branched-chain paraffins exhibit weak cool flames while still exhibiting two-stage auto-ignition behaviour. Therefore, in order to predict whether an auto-ignition event will occur, one needs to know the in-cylinder temperature distribution and history.

The main methods for modelling auto-ignition are detailed chemical kinetic models, reduced chemical kinetic models and empirical induction-time correlations. The most comprehensive of these is chemical kinetics which embodies all the hydrocarbon oxidation processes. This 
is computationally expensive, especially when using it with other models. To overcome this computational difficulty, methods which reduce the number of chemical processes that need to be modelled have been developed. This forms a very large part of the previous and ongoing research in the field and a comprehensive review can be found in [11]. The $\mathrm{PhD}$ thesis produced by Viljoen [14] also provides a comprehensive review of auto-ignition chemistry models.

Simpler models based on empirical induction-time correlations are also widely used. These correlations are derived by matching an Arrhenius function to measured auto-ignition times for given fuel-air mixtures over the relevant pressure and temperature ranges [1]. The ignition delay time, $\tau$, is given by [15]:

$$
\tau=A p^{-n} \exp \left(\frac{B}{T}\right)
$$

$A, n$ and $B$ are fitted parameters that depend on the fuel. The induction-time $(\tau)$ is measured in milliseconds; the absolute pressure $(p)$ in atmospheres.

\subsection{The octane rating of gasoline fuels in the CFR engine}

Practical fuels are blends of paraffins, napthenes, olefins and aromatics. In order to compare different fuel blends, a practical measure of a fuel's resistance to knock is used. This measure is defined by the octane scale and measured according to standard octane rating methods performed using the CFR engine shown in Figure 2.3. The ONs of commercial fuels are measured by comparing it to the onset of knock under the same conditions for a mixture of the PRFs $n$-heptane and iso-octane. 


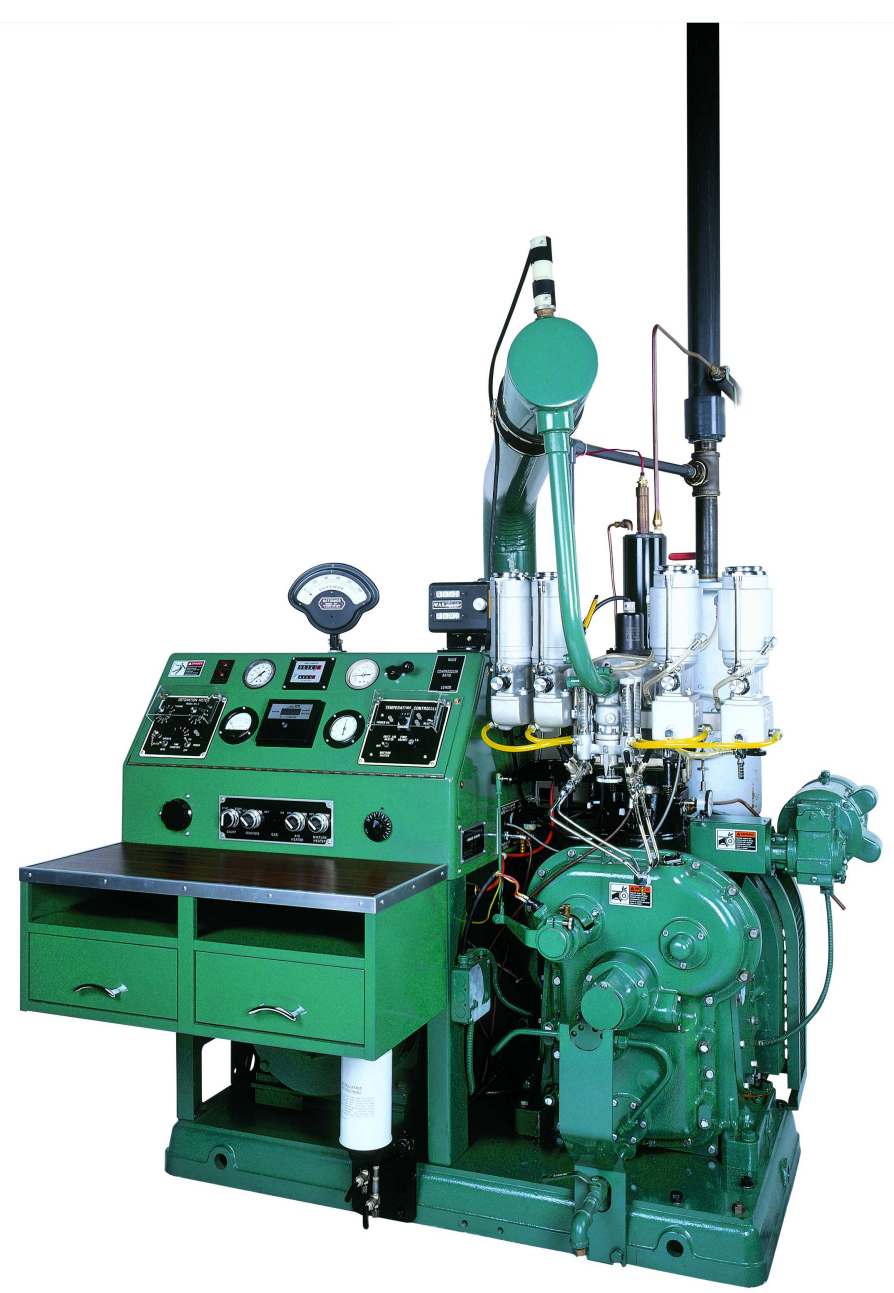

Figure 2.3: The CFR engine [16] 
The CFR engine is a single cylinder carburettor engine which operates at low speeds (600 and $900 \mathrm{rpm}$ ). Detailed information on the engine setup and parameters are given in Table 2.1.

Table 2.1: $C F R$ engine parameters

\begin{tabular}{lcc}
\hline Bore & $B$ & $0.08255 \mathrm{~m}$ \\
Stroke & $L$ & $0.11430 \mathrm{~m}$ \\
Con rod length & $l$ & $0.254 \mathrm{~m}$ \\
Inlet valve opens & IVO & $10^{\circ}$ ATDC \\
Inlet valve closes & IVC & $34^{\circ}$ ABDC \\
Compression ratio & RC & variable \\
\hline
\end{tabular}

One of the important design features of the CFR engine is the inlet valve. This valve (see Figure 2.4 has a shroud that closes off $50 \%$ of the valve curtain area. The purpose of the shroud is to create swirling flow as well as turbulence in the cylinder. The inlet valve is also locked in its orientation, unlike production engine valves that are free to rotate.

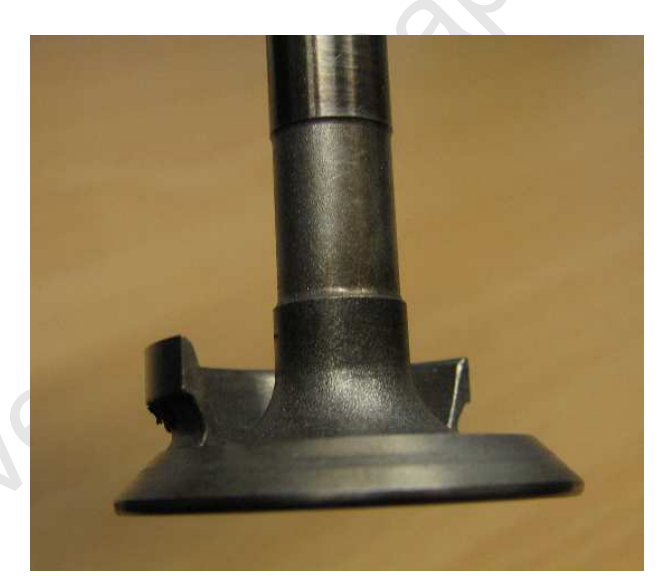

Figure 2.4: CFR inlet valve with shroud [17]

The RON and MON octane rating tests require critical adjustment of the air-fuel mass ratio (AFR) and compression ratio (CR) to maximum knock intensity at any operating condition. The anti-knock quality of a gasoline sample is determined by compatring it with that of primary reference fuel blends.

The two procedures are used to determine the $\mathrm{ON}$ of a fuel and were originally intended to represent the typical on-road operation of a vehicle. The operating parameters for both tests are taken from [5] and shown in Table 2.2. 
Table 2.2: CFR engine operating conditions

\begin{tabular}{lccc}
\hline & & RON & MON \\
\hline Engine speed & {$[\mathrm{RPM}]$} & 600 & 900 \\
Spark timing & {$[\mathrm{BTDC}]$} & $13^{\circ}$ & $19^{\circ}$ to $26^{\circ}$ \\
Inlet air temperature & {$[\mathrm{K}]$} & 325 & 311 \\
Inlet mixture temperature & {$[\mathrm{K}]$} & not specified & 422 \\
\hline
\end{tabular}

The inlet temperature is controlled at (a) and the mixture temperature at (b) as shown in Figure 2.5 below.

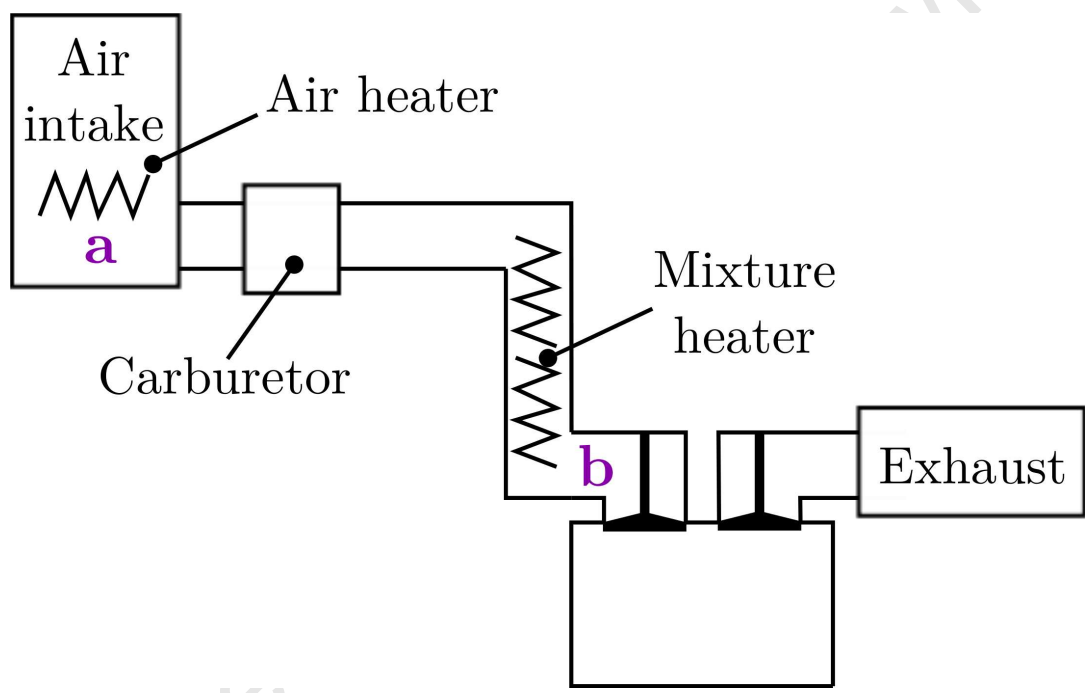

Figure 2.5: Schematic of CFR engine indicating temperature control locations

Combined, these tests provide a means of defining the antiknock quality of a motor fuel for use in vehicles on the road, given by [5]:

$$
\text { Antiknock Index }=\frac{\mathrm{RON}+\mathrm{MON}}{2}
$$

\subsubsection{Octane Sensitivity}

In general RON is higher than MON. The difference between the two is known as the fuel's OS:

$$
\mathrm{OS}=\mathrm{RON}-\mathrm{MON}
$$

The higher RON values are of particular interest and it is believed that evaporative cooling may play a role. In the RON test the air temperature is heated to $325 \mathrm{~K}$, which is measured at a single point. At this temperature, not all the fuel is evaporated in the manifold, and 
thus the possibility of cooling of the mixture exists. This is due to the latent heat required to evaporate the liquid fuel.

Engine modelling can aid in gaining detailed insight into knocking and OS in the CFR engine. Although modern day engines use direct injection methods as opposed to carburettors, the latter type of engine is still being used to rate the $\mathrm{ON}$ of fuels for modern day advanced engines.

\subsection{Engine modelling}

Engine modelling is defined as the development and use of the appropriate combination of assumptions and equations that permit critical features of the process to be analyzed. The primary processes involved are the intake, compression, combustion, expansion and exhaust processes. The process of interest dictates the modelling techniques that are required and often the in-cylinder processes such as combustion, both normal and abnormal (engine knock) are the primary focus.

Modelling of the intake and exhaust processes - also known as manifold models - is important as these provide initial or boundary conditions to more detailed models of in-cylinder processes. The main sub-models for calculating intake and exhaust flows are listed below [18]:

1. Quasi-Steady flow models are often used to calculate the flow into and out of the cylinder through the inlet and exhaust valves. The main aim of these is to provide mass-flow rates into and out of the cylinder.

2. Filling and emptying models are used when the finite volumes of the critical manifold components need to be accounted for. These models allow for the division of a single volume into many sections, with flow restrictions such as throttle valve or inlet valve before, after or in between volumes. Each volume then forms a control volume (CV) with gas at a uniform state and with mass flow rates into and out of it.

3. When the spatial variations in flow and pressure throughout the manifolds are important, gas dynamic models are incorporated. These models calculate the mass, momentum and energy conservation equations for the unsteady, compressible flow in the intake and exhaust. Normally the 1D unsteady flow equations are used, however this can be extended to multi-D flow models which can take into account the 3D effects of the flow in the manifolds.

The overall dynamic characteristics of intake and exhaust flows are modelled relatively accurately using 1D unsteady fluid dynamic models. However, the flow through the ports 
and in the cylinder is inherently unsteady and 3D. Engine models can be developed using 3D CFD, and it is a very powerful tool in predicting gas-flow patterns. Its capability to accurately predict all the real engine processes is, however, still in question. The prediction of fuel spray behaviour is possible, however, its capability to represent real life spray flow is less complete. This is due to fuel sprays consisting of millions of droplets of different sizes. Modelling individual droplets is not feasible due to the huge computational cost associated with it. Instead, statistical averaging is applied to break the large spray into parcels. Additionally, many of the sub-models - such as the wall film models - are based on single droplet research which has not been validated for spray applications where thousands of droplets are interacting with the wall film. Combustion calculations are computationally expensive and the management and optimization of computational expense and accuracy can become challenging. Conventional 0D multi-zone models for combustion are thus still favoured [18].

The in-cylinder processes are generally modelled using $0 \mathrm{D}$ or 1D thermodynamic models combined with a two-zone (or multi-zone) model for the combustion process. The gas species which make up the working fluids in IC engines can usually be treated as ideal gases [19] which are governed by the equation of state for an ideal gas given by:

$$
P V=m R T
$$

$R$ is the specific gas constant. $P, V, m$ and $T$ are the pressure, volume, mass and temperature of the bulk gas respectively.

For the multi-zone model, the combustion chamber is divided into a burnt and unburnt zone. Within each zone, empirical correlations are used to describe thermodynamic processes, and heat and mass transfer occurs between the two zones. Multi-zone models allow for the burnt and / or unburnt zones to be further divided. Since this is a 0D approach, gas properties and state variables such as pressure, temperature and density represent an average over a zone; non-uniformities cannot be accounted for. This also results in the assumption that all the air and fuel is fully and uniformly mixed. Since liquid fuel distributions cannot be accounted for, all the liquid fuel is assumed to form part of the wall film or is fully evaporated [7, 20].

As mentioned in section 2.1.1, auto-ignition events can be modelled using detailed or reduced chemical kinetics or by empirical induction-time correlations. Since auto-ignition is a predominantly localized event, spatial variations in flow properties need to be accounted for. Multi-zone models allow either or both the burnt and unburnt zone to be divided into more zones. The greater the number of zones, the more spatial resolution of properties is possible. In a recent study by Perumal [17, 21], such a multi-zone approach was implemented. 
The combustion chamber was split into many annular zones in order to take into account the spacial variation of unburnt gas properties. Floweday's [22] rate-based global auto-ignition model for reaction modelling and auto-ignition prediction was then applied to each zone respectively. The difficulty arose in assigning initial properties to each individual zone. Experimental temperature measurements or full 3D simulations would be required. The direct measurement of unburnt gas temperatures still proves difficult, because non-invasive in-cylinder measurement techniques are not very accurate. Coherent anti-Stokes Raman Spectroscopy (CARS), for example, measures in-cylinder temperatures to within $50 \mathrm{~K}$ [11]. This uncertainty is very significant as auto-ignition is extremely sensitive to temperature. The use of 3D CFD can thus be a powerful tool if used for this purpose, and, although limited experimental validation of CFD is possible, it is much less expensive and complex than a CARS laser system.

The benefits that full 3D CFD present, for at least some of the processes described above, is evident. Instead of sampling properties at a limited number of positions, the complete spacial and temporal distribution of these properties are available. These temporal distributions can provide better estimates of initial and boundary conditions for thermodynamic models. Spatial variation of properties obtained from CFD solutions can be used in multi-zone models where the number of zones can be optimized to best represent the spatial gradients of properties.

In the current study the spatial distribution of temperature is of primary interest as this dictates the onset of auto-ignition. However, spatial variation in AFR can also provide insight into mixing of the fresh charge and the hot residual exhaust gas (REG) within the cylinder. Most two-zone SI engine models have a burnt and unburnt zone, where each of these zones have a set of properties assigned to them and interaction between the zones can take place. Coupling the outputs of a full 3D CFD model with such a 0D multi-zone auto-ignition model can thus be useful in terms of more comprehensive prediction of auto-ignition and knock in SI engines.

Furthermore, full 3D CFD coupled with a liquid fuel spray model can aid in better understanding the effect that liquid fuel and fuel evaporation has on the in-cylinder temperature distribution. It can provide more information regarding the in-cylinder in-cylinder mixing of the fresh fuel-air mixture and the hot REG under both RON and MON conditions. 


\subsection{Liquid fuel sprays in SI engines}

Sirignano [23] and Sazhin [24, 25, 26, 27] have published many papers pertaining to fuel spray and the modelling thereof. There is also a lot of literature on spray wall interaction and fuel films (for example [28, 29, 30]), but the majority of these only consider single droplets. In a recent review paper by Moreira [31], the extent of usefulness of single-droplet studies was evaluated. The review was mainly focused on IC engines applications. The main findings of this review were that the formulation for the modelling of wall film dynamics is well documented and implemented in commercial CFD codes, but that it is not known how accurately these models represent fuel film flow in engines.

Various models of droplet heating and vaporization exist. Three of these models, which are suitable for implementation in CFD codes, are discussed briefly. Their definitions are taken directly from Sazhin [24]:

1. Models based on the assumption that there is no temperature gradient inside droplets (infinite thermal conductivity of liquid).

2. Models taking into account finite liquid thermal conductivity, but not the re-circulation inside droplets (conduction limit).

3. Models taking into account both finite liquid thermal conductivity and the re-circulation inside droplets via the introduction of a correction factor to the liquid thermal conductivity (effective conductivity models).

More complex models based on vortex dynamics and full solutions to the Navier-Stokes equations exist. These are not considered as their implementation becomes too complex when considering thousands of droplets in a spray. The models mentioned do not include droplet breakup, coalescence or heating and vaporization on heated surfaces. The infinite thermal conductivity model is the simplest model and is used by most commercial CFD packages.

\subsubsection{Heat transfer}

There are two main processes that need to be accounted for when considering droplet heating. Firstly, during heating, the droplet might deform in the air stream. Secondly, there may be a non-uniform distribution of temperature at the droplet surface at any given time. When modelling a large number of droplets, as found in spray applications, these processes can lead to computational difficulty and substantial errors. A reasonable compromise between accuracy and computational efficiency is found via these two main assumptions for the modelling of droplet heating [24]:

- The droplet remains spherical even in the process of its movement. 
- The temperature over the whole droplet surface is the same (although it can vary with time).

In the case of droplet heating where there is no temperature gradient inside the droplet, the temperature of the droplet can be found from an energy balance:

$$
m_{d} c_{d} \frac{d T}{d t}=h A_{d}\left(T_{\infty}-T_{d}\right)+\text { radiation }
$$

where:

$$
\begin{array}{rll}
m_{d} & - \text { droplet mass }[\mathrm{kg}] \\
c_{d} & - \text { droplet specific heat }[\mathrm{J} / \mathrm{kgK}] \\
A_{d} & - \text { droplet surface area }\left[\mathrm{m}^{2}\right] \\
T_{\infty} & - \text { temperature of the bulk gas }[\mathrm{K}] \\
T_{d} & - \text { droplet temperature }[\mathrm{K}]
\end{array}
$$

The heat transfer coefficient, $h$, is found from the dimensionless Nussult number correlation given by [24]:

$$
N u=\frac{h L}{k}=2+\beta_{c} \operatorname{Re}^{\frac{1}{2}} \operatorname{Pr}^{\frac{1}{3}}
$$

The Prandtl number is based on specific heat capacity $\left(c_{p}\right)$, dynamic viscosity $(\mu)$ and thermal conductivity $(k)$ of the gas phase given by:

$$
\operatorname{Pr}=\frac{c_{p} \mu}{k}
$$

The Reynolds number is based on the droplet diameter $(d)$, the relative velocity $\left(v_{r}\right)$ between the droplets and surrounding gas, and the gas density $(\rho)$ and viscosity $(\mu)$ given by:

$$
R e=\frac{\rho v_{r} d}{\mu}
$$

Models for droplet heating based on this approach are known as the Spalding model as given in [23].

For time-dependent cases, changes in the surrounding gas' temperature and flow velocity need to be accounted for in the heat transfer calculations. The assumption that the flow is fixed is often used, since the time over which flow parameters change significantly is much less than the time required for significant droplet heating to occur 24]. CFD calculations are performed over small time steps over which the variations of gas and liquid parameters such as thermal conductivities, specific heat capacities and densities - can be ignored within a time step. This simplification allows the properties to be updated from time step to time step [24]. 


\subsubsection{Mass transfer}

Evaporation is the transport of the saturated vapour to the surface of the droplet and the change of phase at the surface. The driving force is the difference in concentration of the droplet vapour between the droplet surface and the free stream [32]. Most models assume that the fuel vapour in the vicinity of the droplet surface is always saturated. Hence, the rate of fuel evaporation is equal to the rate of fuel diffusion from the droplet surface to the gas. Such models are known as hydrodynamic models. These models do not take detailed chemical kinetics into account as these are mathematically complicated. For the hydrodynamic models, the difference in vapour concentration at the droplet surface and the bulk gas acts as the gradient driving mass diffusion [24].

Similar to the convection heat transfer coefficient, $h$, the mass transfer coefficient, $k$, is used to describe evaporation. In the general case, $k$ can be found using the Sherwood number correlation given by [33]:

$$
S h=\frac{k d}{D_{i, m}}
$$

Where $D_{i, m}$ is the diffusion coefficient of vapour in the bulk gas with units of $\mathrm{m}^{2} \cdot \mathrm{s}^{-1}$. The correlation for $S h$ for moving evaporating droplets is obtained from [33]:

$$
S h=2.0+0.6 R e_{d}^{\frac{1}{2}} S c^{\frac{1}{3}}
$$

The Schmidt number $S c$ is the ratio of kinematic viscosity of the gas phase $\left(\frac{\mu}{\rho}\right)$ and mass diffusivity of the vapour phase given by:

$$
S c=\frac{\mu}{\rho D_{i, m}}
$$

This is known as the Ranz-Marshall correlation [32].

The molar flux of droplet vapour into the surrounding gas, $N_{i}$, is related to the difference in vapour concentration at the droplet surface, $C_{i, s}$, and the bulk gas, $C_{i, \infty}$, by [33]:

$$
N_{i}=k\left(C_{i, s}-C_{i, \infty}\right)
$$

$C_{i, s}$ is evaluated by assuming that the partial pressure of the vapour at the interface is equal to the saturated vapour pressure, $p_{\text {sat }}$, at the droplet temperature, $T_{d}$ :

$$
C_{i, s}=\frac{p_{s a t}\left(T_{d}\right)}{R T_{d}}
$$

$R$ is the universal gas constant. $C_{i, \infty}$ is known from the transport equation for species $i$ : 


$$
C_{i, \infty}=X_{i} \frac{p}{R T_{\infty}}
$$

where:

$X_{i} \quad$ - bulk mole fraction of species $i$

$p \quad$ - local absolute pressure

$T_{\infty}$ - local bulk temperature in the gas

If the molar flux of vapour equation is multiplied through by the molar mass and the droplet surface area, this equation reduces to the Maxwell equation [33]:

$$
\dot{m}=A_{d} k\left(\rho_{i, s}-\rho_{i, \infty}\right)
$$

\subsubsection{Thermo-physical properties required for spray evaporation modelling}

The liquid phase thermodynamic properties required are provided in Table 2.3 at either the reference or boiling temperature. These properties were taken from [34].

Table 2.3: Thermophysical properties 34]

\begin{tabular}{lccc}
\hline & & Toluene & Iso-octane \\
\hline Liquid density & {$\left[\mathrm{kg} \cdot \mathrm{m}^{-3}\right]$} & 866 & 688 \\
Specific heat & {$\left[\mathrm{J} \cdot \mathrm{kg}^{-1} \cdot \mathrm{K}^{-1}\right]$} & 2088 & 2088 \\
Thermal conductivity & {$\left[\mathrm{W} \cdot \mathrm{m}^{-1} \cdot \mathrm{K}^{-1}\right]$} & 0.151 & 0.137 \\
Binary diffusivity & {$\left[\mathrm{m}^{2} \cdot \mathrm{s}^{-1}\right]$} & $8.8 \mathrm{e}-6$ & $6.65 \mathrm{e}-06$ \\
$\mathrm{~T}_{\text {boiling }}$ & {$[\mathrm{K}]$} & 384 & 372.4 \\
Latent heat at $\mathrm{T}_{\text {boiling }}$ & {$\left[\mathrm{J} \cdot \mathrm{kg}^{-1}\right]$} & 363431 & 270000 \\
\hline
\end{tabular}

Density, specific heat, thermal conductivity and binary diffusivity are all given at atmospheric conditions. LHV is given at the liquid phase boiling temperature.

The binary diffusivity of the fuel, also known as the mass diffusivity, is used to calculate the mass transfer coefficient, $k$. The binary diffusivity of the droplet particle, $D_{i, m}$, can be defined as a function of temperature. Also, $D_{i, m}=D_{A B}$, where $D_{A B}$ is the diffusion coefficient of vapour in the bulk gas $\left(\mathrm{m}^{2} \cdot \mathrm{s}^{-1}\right)$ [33].

$D_{A B}$ is found from the Fuller equation, which has shown the best performance over a wide range of substances and is recommended for use by Poling et al. [35]. The Fuller equation is given by: 


$$
D_{A B}=\frac{0.00143 T^{1.75}}{P M_{A B}^{\frac{1}{2}}\left[\left(\Sigma_{v}\right)_{A}^{\frac{1}{3}}+\left(\Sigma_{v}\right)_{B}^{\frac{1}{3}}\right]^{2}}
$$

where:

$$
\begin{array}{cl}
\mathrm{M}_{A B} & -2\left[\frac{1}{M_{A}}+\frac{1}{M_{B}}\right]^{-1} \\
\mathrm{M}_{A}, \mathrm{M}_{B} & - \text { molecular weights of A and } \mathrm{B},\left[\mathrm{g} \cdot \mathrm{mol}^{-1}\right] \\
\Sigma_{v} & - \text { sum of atomic diffusion volumes }
\end{array}
$$

$\Sigma_{v}$ is found for each component by summing atomic diffusion volumes as found in [35]. The authors report an average absolute error of $4 \%$ when using this equation.

Additionally to the properties mentioned above, the saturation vapour pressure of the liquid phase must be defined over the entire temperature range. Its definition is critical to the evaporation process as it is used to calculate the vapour concentration at the droplet surface. An upper limit on the amount of fuel that can evaporate occurs when the air becomes saturated. For pure component fuels this is when the partial pressure of the fuel equals its saturation pressure.

The two most widely used equations for calculating vapour pressure are the Wagner and Antoine equations. In [36] the Wagner equation was used and showed good results. Empirical constants for a wider range of hydrocarbons are however available for the Antoine equation give by [37]:

$$
\log _{10} P_{v p}=A-\frac{B}{T+C-273.15}
$$

$A, B$ and $C$ are empirical constants determined experimentally for each fluid. The benefit of using the Wagner equation is that extrapolation to lower or higher temperatures is possible. Extrapolation of the Antoine equation is not reliable and hence care must be taken in ensuring that it is used within the stated temperature limits. Constants for the Antoine equation are given for iso-octane and various other fuels over a temperature range of roughly $275 \mathrm{~K}$ to 400 $\mathrm{K}$ in [37]. The droplet temperatures are not expected to be outside of these limits. 


\subsection{Current questions surrounding OS, knock and evaporative cooling}

Two schools of thought exist regarding OS in the CFR engine. Many papers have attributed it to the chemical characteristics of the fuels, and there is an abundance of literature available that study knock using detailed chemical kinetics [11, 9, 10]. Others have concluded that the difference in test conditions gives rise to evaporative cooling effects in the RON test which explains the better anti-knock measurement (higher RON value). This evaporative cooling hypothesis was studied in a masters thesis by Moran [8] at the University of Cape Town (UCT) and later followed up with research work in the industry with Taylor [7]. Combining physical and chemical effects would be the best way to study knock in SI engines, and this was done in the work by Stauch and Maas [38]. Their study did however not account for the transient engine environment and operating conditions, nor the geometrical characteristics and flow fields. Bradley and Head [39] conducted a study to establish the relationship between autoignition delay times and $\mathrm{ON}$.

The evaporative cooling effect investigated in [8] was carried out looking only at the inlet manifold. The model was based on the inlet manifold of the CFR engine. A 1D model was used to model the fuel spray, evaporation and wall film flow. Steady state conditions were assumed, with the model run for representative flow velocities. The computer model simulations were compared to experimentally measured temperatures in a test pipe. The main conclusion of this work was that OS can almost fully be explained by evaporative cooling effects and that the MON method ranks fuels by their chemical resistance to knock, while the RON tests give fuels an advantage via evaporative cooling of the unburnt gas. The transient nature of intake and compression in SI engines could not be accounted for in this study and this remained an area of interest. Pulsating flow and variation in air speed can only be fully included in a transient simulation.

In a later paper by the same author [7], an additional model was created to investigate the unburnt gas temperature in the cylinder under RON conditions. One of the focuses of this study was liquid fuel entering the cylinder and its effect on unburnt gas temperatures. The study assumed that all of the liquid fuel entering the cylinder would deposit on the chamber walls where it would gain energy for vaporization from the hot metal surfaces, and not the surrounding gas. This assumption also implied that the liquid evaporating on the chamber surfaces would not affect the unburnt gas temperatures much. The main conclusion was that fuel evaporative cooling plays an important role in fuel anti-knock performance and OS. It should be noted that, if the latent heat transfer is not cooling the unburnt gas, it is cooling the chamber walls. As the walls are cooled, less heat is available to vaporise the liquid film, and 
hence more energy would be required from the surrounding gas. This important observation was not made in this study. The assumptions made in the study pose two main issues:

1. If all the liquid does not impinge on the wall, then the liquid remaining in free flow will take its heat of vaporisation from the surrounding gas.

2. If the wall film cools the hot metal surfaces as it evaporates, then a stage would be reached where the energy for vaporization will have to be sourced from the gas phase and hence can still have a cooling effect.

This hypothesis has received much attention from the SAFL research group with much of the work being refocused on octane tests and OS. One such a study done by Swarts and Yates [6] aimed at quantifying the in-cylinder fuel evaporation based on the polytropic character of compression. Swarts later produced a Ph.D. thesis on the insights relating to octane rating and the underlying role of auto-ignition [13] where knock in the CFR was studied in detail.

This issue of OS did not only receive interest at the SAFL. Another study done at the University of Oxford by Wyszynski et al. [36] aimed at correlating the extent of evaporative cooling in the cylinder by looking at improvements in volumetric efficiency (VE) between port and direct fuel injection. The further the injection point from the cylinder, the less fuel will enter the cylinder as liquid, and thus the less in-cylinder fuel evaporative cooling will take place. The findings indicated a $9 \%$ increase in VE when changing from port to direct injection. A further 3\% was gained when adding $10 \%$ methanol to the fuel blend. These differences were then used to calculate the extent of cooling by evaporation and comparing it to the maximum evaporation potential of the fuel. The maximum evaporation potential was calculated using a model that assumed that all the LHV went to cooling the gas and none to the walls and other sources of thermal energy. It was found that the VE improvement was generally between $50 \%$ and $70 \%$ of the maximum possible improvements calculated according to the assumption that all the evaporative cooling is applied to the unburnt gas and none to the walls. This improvement is rather substantial as it indicates that unburnt gas cooling does in fact play a role within the cylinder. Although these investigations were not done on a CFR engine, it again demonstrates the effect that evaporative cooling can have.

In [40] Chen et al. combined the discrete particle method (DPM) for tracking sprays with the 1D gas flow code WAVE. The aim of this model was to study the behaviour of the fuel spray and wall film as these are key factors in determining air-fuel mixing and hence combustion and emissions. This study claimed that 3D CFD calculations are inefficient on computing time and cost, since the transient engine process persists over hundreds of engine cycles. The 1D code included impingement on flow obstacles such as the port and cylinder walls, as well as the fuel film formation. Two important points regarding mixing time were also made: 
1. The further the injection point from the inlet, the more charge preparation time there is and the more mixing time is available.

2. The further the injection point from the inlet, the longer it takes for the air-fuel mixture to reach the cylinder. This retards the response to fuel equivalence ratio $(\lambda)$ changes within the cylinder.

The mixing time during MON is longer than RON1, however during MON the increased mixing time would result in more homogeneous charge preparation before it reaches the cylinder. Since the transient process is too computationally expensive to be used in 3D CFD, Chen et al. [40] suggested the use of a 1D model for gas flow simulations with liquid fuel spray. Hsiao 41] produced a Master's thesis at SAFL where flame propagation in the CFR engine under knocking and non-knocking conditions was studied. The flame propagation and combustion was modelled with a two-zone engine model. This study also used CFD to study the incylinder flow set up prior to combustion, and this information aided in making assumptions regarding the flame shape. In order to determine spatial variations in in-cylinder temperature and charge, 3D CFD would be required, especially when the focus of the current work is to determine the spatial variations of temperature within the cylinder.

In [42], Liu and Karim developed such a 3D CFD simulation, which also included detailed chemical kinetics, for the Homogeneous Charge Compression Ignition (HCCI) engine. The aim of the study was to investigate the combustion characteristics of HCCI engines, and how changes in variables such as $\mathrm{CR}$ and combustion chamber wall temperature affect auto-ignition and combustion characteristics of HCCI engines. A CFR engine was used and modified to act as an HCCI engine. Although a CFR engine operates as a SI engine, while a HCCI engine operates as a Compression Ignition (CI) engine, this study is useful in the context of the current work, as it investigated the in-cylinder temperature distribution set up during compression. In the HCCI engine, the liquid fuel is injected directly into the cylinder, allowing for evaporative cooling of the in-cylinder gas. Based on the temperature profiles observed in the combustion chamber, it was concluded that the combustion chamber wall temperature greatly influences the auto-ignition timing and its location. For very hot wall temperatures, the temperature profiles indicated that the hotter gas is found in the regions adjacent the walls, and not in the central regions of the combustion chamber. This finding further demonstrates the importance of knowing the in-cylinder temperature distribution in predicting knock.

In 20] a 1D droplet evaporation model was developed and used to estimate the fraction of incylinder liquid fuel volume that vaporizes before impacting on the cylinder walls or piston top.

\footnotetext{
${ }^{1}$ Although the MON engine speed is faster than that of RON, the extra heating manifold increases the distance the fuel and air travels prior to entering the cylinder.
} 
The droplet characteristics were measured in the valve vicinity using Phase Doppler Particle Anemometry (PDPA) and Planar Laser-Induced Fluorescence (PLIF) imaging. Measured port and valve temperatures as well as in-cylinder gas temperature and the mean air velocity in the valve gap as obtained from a $0 \mathrm{D}$ engine simulation code were used to account for the transient environment in the cylinder during each cycle. The initial size and velocity of the droplets in the vicinity of the inlet valve was found using the experimental setup which can be found in [43. A transparent single cylinder square-piston engine was used with PDPA measurements for the droplet characteristics. The initial temperature of the droplets at entry to the cylinder, however, was estimated to be the average of the valve and the port wall temperatures, which were measured using the experimental engine. Injection of the fuel occurred in the port close to the inlet valve. For the computational model, the engine cylinder was modelled with a circular geometry, with the cylinder bore such that the round cylinder cross-section equalled the square one used in the experimental setup. The in-cylinder air temperature and air velocity in the valve gap during intake were calculated using a $0 \mathrm{D}$ engine cycle simulation.

One of the main findings of this study was that the initial droplet temperature had a significant influence on the rate of droplet evaporation, compared to that of the surrounding gas which was found to be negligible. The latter observation was attributed to the time that the droplets spent in the cylinder before impacting on the combustion chamber walls. This period was not greater than the heat-up period required before the droplets reached the wet-bulb temperature2. Increased heat transfer through increased cylinder temperatures did not speed up evaporation. However, increased initial temperatures shortened the heat-up period of the droplets and hence less heat up time was required and evaporation set on sooner. Limitations of this study are listed below:

- Droplet interactions with spatial features of the cylinder flow field were not taken into account. The model could not account for the spatial variation of in-cylinder flow, which is believed to carry droplets with diameters smaller than approximately $10 \mu \mathrm{m}$ with it.

- Droplet interactions within the spray were not modelled.

- The initial temperatures of the droplets were found to be significant, however were estimated to be the average of the wall and gas temperatures.

- The highly transient and 3D flow through the port was calculated using a 0D engine simulation.

The most interesting observation made in this study was that the droplet travelling time within the cylinder was short and that it could be expected that droplet heat up would

\footnotetext{
${ }^{2}$ This is the temperature a droplet is heated to before evaporation starts taking place
} 
still be occurring at the time the droplets impact the cylinder or piston walls, implying that evaporative cooling within the cylinder is limited. However, with the swirling flow field which is set up in circular bore engines - especially in the CFR engine where the shrouded inlet valve directs the flow circumferentially along the cylinder boundaries - this travelling time may be increased, allowing additional evaporative cooling of the mixture to take place. These findings also become less applicable when considering carburettor fuel intake in the CFR engine upstream of the inlet port. The effect of the high velocities through the inlet valve need also be considered as the advective heat transfer can increase the rate of evaporation of the fuel liquid further.

In [9], Mehl et al. discussed the possibility and limits of two zone models for SI engines using a quasi-D, two-zone combustion model coupled with a 1D thermo-fluid dynamic code, in which detail kinetic schemes in the unburnt mixture was introduced. The work was focussed on the octane rating in the CFR engine and was aimed at developing a knock prediction model for SI engines as a function of the engine characteristics and operating conditions as well as the fuel composition. It was found that the coupling of chemical kinetics with quasi-D, two zone model for SI engines allowed a good description of the knocking propensity of different hydrocarbons. The study also provided a means of predicting a fuel's knock propensity making it very useful in octane rating.

This work was later extended in [10] to the prediction of knock in 4-cylinder, naturally aspirated engines. It was once again shown how a coupled chemical kinetic 1D, twozone model for SI engines produces a very good description of the knocking propensity of different hydrocarbon mixtures. Mehl et al. also stated that CFD models can provide useful information about the flow pattern inside the combustion chambers or ducts, but that overall engine performance is better optimized using 1D models.

The problem with 0D and also 1D approaches, however, is that assumptions often need to be made about the state of the in-cylinder contents and distribution of its properties. For example, in [6, 20] the assumption was made that all the liquid fuel entering the cylinder impinges on the walls of the combustion chamber. In [21], which was discussed in Section 2.3. Perumual incorporated a multi-zone model with multiple annular zones making up the combustion chamber volume. This was done so that an initial temperature profile could be applied to the combustion chamber at the start of compression. Assumptions had to be made regarding the shape and size of this temperature profile. Different initial profile shapes were studied, however under the further assumption that these profiles were symmetrical about the cylinder axis. Since the current work is focussed on the fuel evaporation dynamics and its effect on the in-cylinder temperature distribution, such assumptions become particularly 
limiting and full 3D CFD is required.

\subsection{Summary}

The octane rating of fuels used in SI engines requires further study due to the effect of OS on fuel anti-knock performance. It is becoming increasingly evident that conventional quasi-D thermodynamic models should be coupled with 3D fluid dynamic models for this purpose. 3D CFD can be especially useful in determining spatial variations of in-cylinder initial conditions and boundary conditions and can be a powerful tool in this regard when used with the knowledge of its flaws and limitations. 


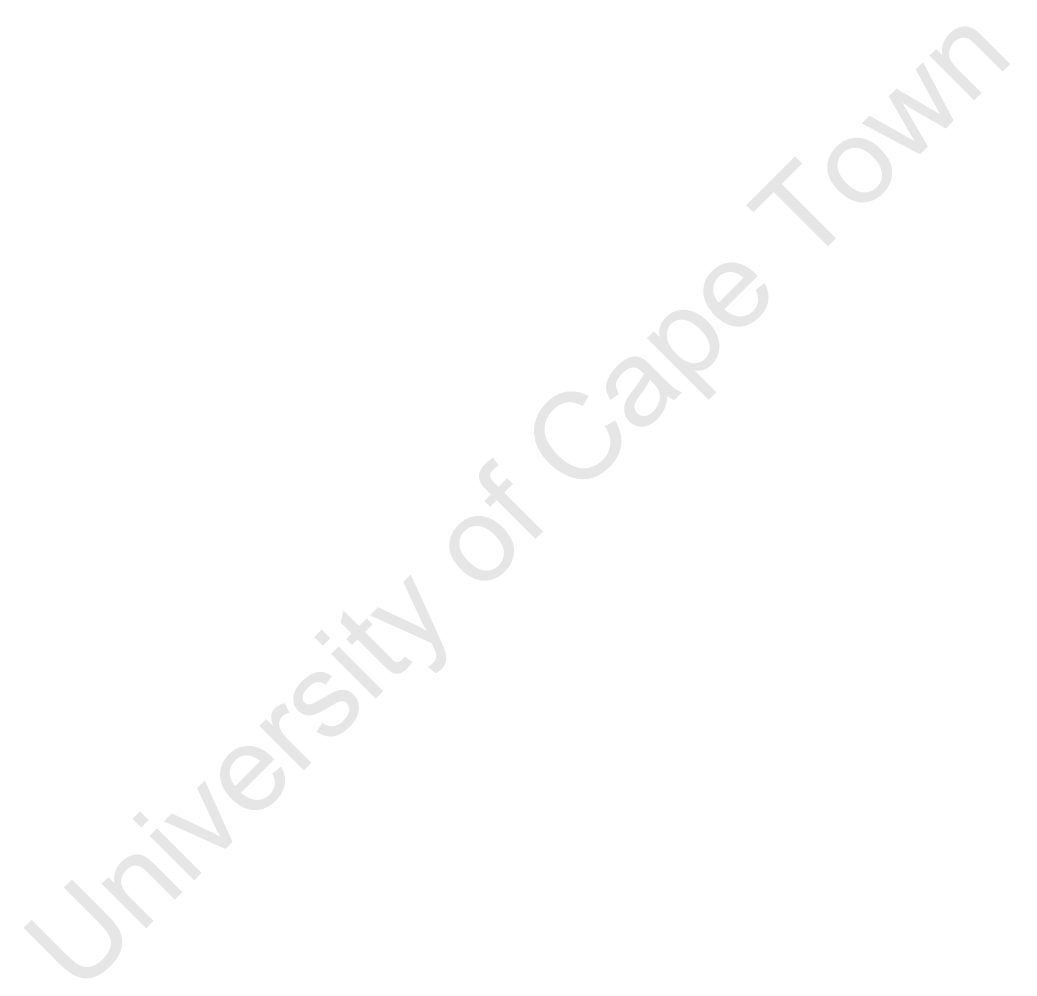




\section{Computational Fluid Dynamics}

This chapter provides a brief description of the theory involved in Computational Fluid Dynamics (CFD), with the emphasis on aspects relevant to this thesis. The theory is also linked to the commercial software packaged that were used in this thesis. The commercial CFD code FLUENT $T^{\mathrm{TM}}$ was used for all flow calculations, while the commercial pre-processing package GAMBIT ${ }^{\mathrm{TM}}$ was used to for geometry and mesh generation.

\subsection{Numerical modelling of the continuous phase fluid flow}

CFD is a method used to analyze fluid flow, heat transfer and other related phenomena using a computer. The equations that govern these phenomena are solved by breaking the flow domain into many discrete CVs. Each equation is solved for each CV. CFD is used over a wide range of applications, with IC engines being a very popular one. The engine geometry is created during the pre-processing phase. Once a suitable computational grid is created and the boundary conditions (BCs) defined, the flow problem can be solved. The user steps through these stages in a structured manner by setting up, solving and finally analysing the fluid flow problem and solution.

\subsubsection{Pre-processing}

At this stage, the flow problem is set up for the solver. It is essential that the problem be set up correctly, as it affects the final solution greatly. The flow problem is set up by creating the geometry that represents the computational domain. This domain must then be meshed by dividing it into an appropriate number of smaller CVs, or elements. Different mesh elements can be used depending on the complexity of the geometry. These are shown in Figure 3.1 


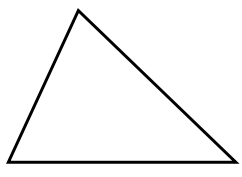

(a) tri

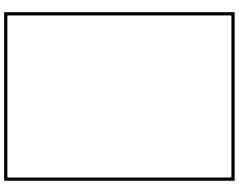

(b) quad

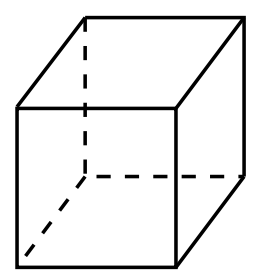

(c) hex

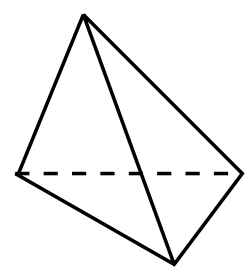

(d) tet

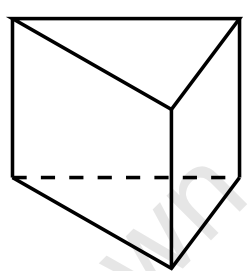

(e) wedge

Figure 3.1: Types of mesh elements

Each element has a node associated with it where the solution to a flow problem is determined. The accuracy of a CFD solution and the time it takes to obtain a solution are thus strongly dependent on the number of elements, and hence nodes, in the mesh. Often more than half the time spent on a CFD analysis is spent in the pre-processor. It is therefore important to find a compromise between the required accuracy and the computational power available.

Incorrect solutions or 'failure to solve' (divergence) often occur due to poor meshing quality. Structured meshes 1 enable the user to control the number of cells in a block of mesh, as well as their placement. Unstructured meshes adapt to the geometry being meshed and, although it can be controlled to some extent2, good mesh quality is not ensured or even always possible.

The general meshing strategy that should be followed is to have a fine, structured mesh (see Figure 3.2a in regions where there are steep variable gradients, and a coarser mesh in regions where the flow is relatively unchanging. The mesh must be fine enough to capture these steep gradients, as well as phenomena such as vortices. The mesh cannot resolve flow phenomena which are smaller than a single element. It is also useful to partition the computational domain into smaller segments to allow for easier meshing. This is useful when a fine, structured mesh is required in one region, but a coarse mesh suffices in another region. An intermediate block, usually consisting of an unstructured mesh (see Figure $3.2 \mathrm{~b}$ ), is then required to link the elements to the fine mesh to the coarse mesh (see Figure $3.2 \mathrm{C}$ ).

\footnotetext{
${ }^{1}$ The term structured here refers to a mesh containing four-sided (paralellogram) elements for the $2 \mathrm{D}$ case. For the 3D case, these elements would be six sided (paralelliped).

${ }^{2}$ Size functions and edge meshing which incorporates grading can be used to control unstructured meshes.
} 


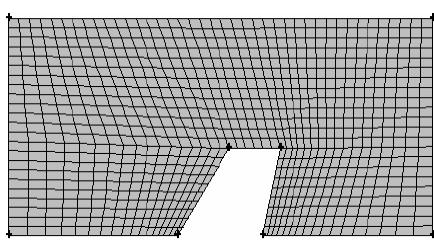

(a) Structured mesh 44,

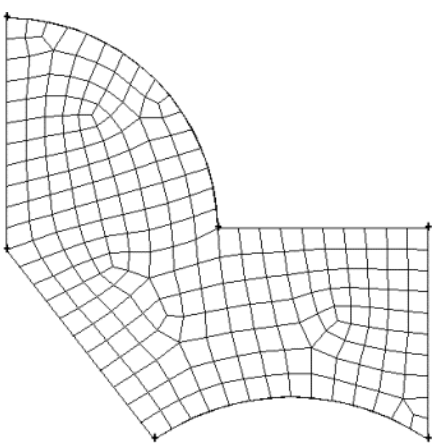

(b) Unstructured mesh 45]

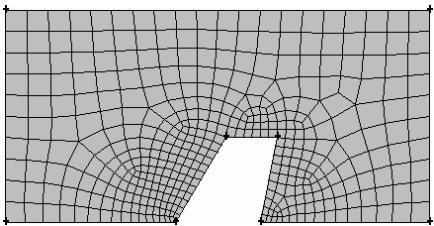

(c) Mesh growth from fine to coarse elements [4]

Figure 3.2: Types of face meshes

The mesh quality should always be checked and, if it is not sufficient, the region containing the poor elements should be remeshed. Various methods for assessing mesh quality exist. The skewness of the mesh elements are often used to indicate the quality of the mesh. All elements are graded between 0 and 1 according to the convention shown in Figure 3.3 .

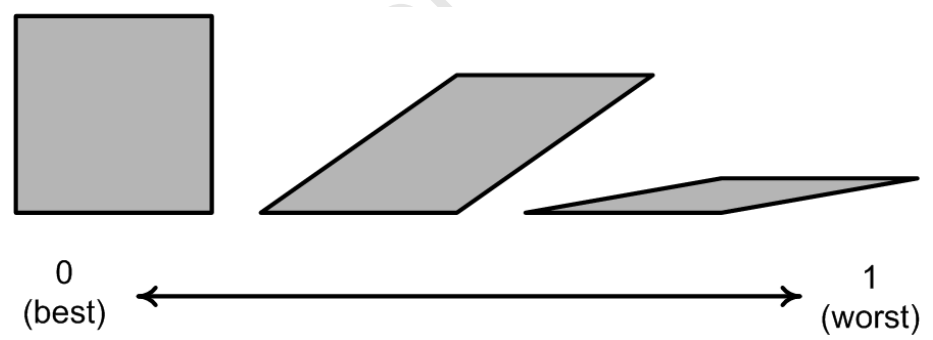

Figure 3.3: Skewness range for quadrilateral elements

The definition changes slightly for 3D elements, however the principals remain the same. The skewness of a cell is calculated based on the following general equation [46]:

$$
\text { skewness }=\max \left[\frac{q_{\max }-q_{e}}{180^{\circ}-q_{e}}, \frac{q_{e}-q_{\min }}{q_{e}}\right]
$$

where:

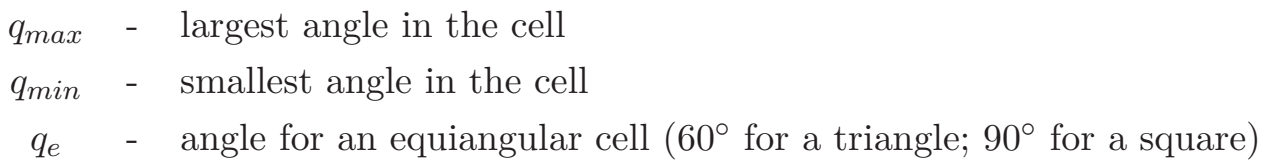

The skewness for all cells should be below 0.85 [44. In the regions of interest, this element skewness should be closer to 0 , and hence structured meshing is generally used in these regions. 


\subsubsection{Governing equations}

The continuous phase fluid flow is governed by the conservation equations for mass, momentum and energy.

The continuity equation states that no mass can be created or destroyed. The rate of increase of mass in the fluid volume is equal to the net rate of flow of mass into the fluid volume. This can be expressed mathematically as:

$$
\frac{\partial \rho}{\partial t}+\operatorname{div}(\rho \mathbf{u})=0
$$

The first term is the time rate of change of the density of the fluid in the fluid element. The second term describes the net flow of mass out of the fluid element across its boundaries and is called the convective term [47].

Newton's second law states that the rate of change of momentum of a fluid particle is equal to the sum of the forces acting on the particle. The conservation of momentum in the flow is described by the Navier-Stokes equations, which, in the $x$ direction and for compressible flow, is given by [47]:

$$
\frac{\partial(\rho u)}{\partial t}+\operatorname{div}(\rho u \mathbf{u})=-\frac{\partial p}{\partial x}+\operatorname{div}(\mu \operatorname{grad} u)+S_{M_{x}}
$$

The term on the left hand side is the rate of change of $x$-momentum of the fluid element. The terms on the right hand side are the forces in the $\mathrm{x}$-direction on the element due to surface stresses plus the rate of increase of $x$ - momentum due to sources. The $y$ - and $z$ - momentum equations have an identical form with the $x$ component of the velocity $(u)$ replaced by the $y(v)$ or $z(w)$ component of the velocity vector, $\mathbf{u}$.

The energy equation is derived from the first law of thermodynamics which states that the rate of change of energy of a fluid element is equal to the rate of heat addition to the fluid element plus the rate of work done on the element, given by:

$$
\frac{\partial(\rho i)}{\partial t}+\operatorname{div}(\rho i \mathbf{u})=-p \operatorname{div}(u)+\operatorname{div}(k \operatorname{grad} T)+\Phi+S_{i}
$$

The dissipation function $\Phi$ describes all the effects due to viscous stresses in the energy equation [47].

The governing equations described previously contain four thermodynamic variables namely density, $\rho$, pressure, $p$, specific internal energy, $i$, and temperature, $T$. These variables are related via the equations of state which are given by $p=f(\rho, T)$ and $i=f(\rho, T)$. For an 
ideal gas the following equations of state are used:

$$
\begin{aligned}
p & =\rho R T \\
i & =c_{v} T
\end{aligned}
$$

For compressible fluids the equations of state provide linkage between the energy equation and the momentum and mass conservation equations [47].

The conservation of momentum equations are known as the Navier-Stokes equations. The Navier-Stokes equations, together with the continuity equation, the energy equation and the equations of state, form the governing equations for fluid flow. Together, these equations form a set of coupled, non-linear partial differential equations (PDEs) that cannot be solved analytically. Numerical techniques are therefore required to solve these equations. This is done by discretising these equations using the Finite Volume Method (FVM). The conservation laws are then solved in each cell.

\subsubsection{The Finite Volume Method for fluid flow}

The FVM is used to solve the equations governing fluid flow. The first step in this numerical algorithm is to integrate the Navier-Stokes equations over each of the CVs within the domain. The resulting integral equations are then discretised into a set of algebraic equations which can then be solved by an iterative method.

The CV integration mentioned above is unique to the FVM, and results in statements that express the conservation of the relevant properties at each finite cell. A general flow variable within a $\mathrm{CV}$ can be expressed as a balance between the various processes which tend to increase or decrease it [48]. The governing equations share many commonalities and with the introduction of a general variable, $\phi$, the conservative form of all fluid flow equations, including scalar quantities such as temperature and species concentrations, can be written in this general form:

$$
\frac{\partial(\rho \phi)}{\partial t}+\operatorname{div}(\rho \phi \mathbf{u})=\operatorname{div}(\Gamma \operatorname{grad} \phi)+S_{\phi}
$$

This becomes the transport equation for a property, $\phi$, and in words states that:

$$
\left|\begin{array}{c}
\text { Rate of change } \\
\text { of } \phi \text { in the } \\
\text { CV w.r.t. time }
\end{array}\right|=\left|\begin{array}{c}
\text { Net rate of } \\
\text { increase of } \phi \\
\text { due to convection }
\end{array}\right|+\left|\begin{array}{c}
\text { Net rate of } \\
\text { increase of } \phi \\
\text { due to diffusion }
\end{array}\right|+\left|\begin{array}{c}
\text { Net rate } \\
\text { of creation } \\
\text { of } \phi
\end{array}\right|
$$

What is useful here is that there is a clear relationship between the numerical algorithm and the underlying physical principle. Convection refers to transport due to fluid flow, and 
diffusion to transport due to variations of $\phi$ from point to point. Source and / or sink terms also arise and are associated with the creation or destruction of $\phi$ respectively. FLUENT ${ }^{T M}$ has discretisation techniques that can deal with all of these phenomena, including the rate of change with respect to time.

The underlying physical phenomena are complex and non-linear, and thus iterative solution approaches are used to solve the algebraic form of the Navier-Stokes equations. The most popular procedures are the Tri-Diagonal Matrix Algorithm (TDMA) line-by-line solver and the Semi-Implict Pressure Linked Equations (SIMPLE) algorithm. The latter ensures the correct linkage between the velocity and the pressure [48]. The SIMPLE method is explained in more detail in Section 3.1.6.

\subsubsection{Reynolds-averaged Navier-Stokes Equations}

Turbulent flows are characterized by fluctuating velocity fields which, in turn, cause transported quantities such as momentum, energy and species concentration to fluctuate as well. These fluctuations are often small in scale and impractical to simulate directly. Instead, the instantaneous governing equations are time-averaged or ensemble-averaged to remove the resolution of small scales. This results in a modified set of equations that are less expensive to solve computationally, but add additional unknowns that need to be solved in terms of known quantities [49].

In 1895, Reynolds presented a first approximate method for modelling turbulence, known as Reynolds Averaging. Here the flow variables are broken into two components: the mean and fluctuating terms [50], given by:

$$
u=U+u^{\prime} \quad v=V+v^{\prime} \quad w=W+w^{\prime} \quad p=P+p^{\prime}
$$

Where $U, V$ and $W$ represent the time-averaged $x, y$ and $z$ velocities, and $P$ the time-averaged pressure. The fluctuating terms are indicated with an accent: $u^{\prime}, v^{\prime}, w^{\prime}$ and $p^{\prime}$. The mean values are generally of most interest to engineers, hence the governing transport equations are solved for these values. This time averaging results in the Reynolds-averaged NavierStokes Equations (RANSE). For the 3D, incompressible case, the RANSE have the following form [50]:

$$
\begin{aligned}
\frac{\partial U}{\partial t}+\operatorname{div}(U \mathbf{U})= & -\frac{1}{\rho} \frac{\partial P}{\partial x}+\nu \operatorname{div}(\operatorname{grad})(\mathrm{U}) \\
& +\frac{1}{\rho}\left[\frac{\partial\left(-\rho \overline{u^{\prime 2}}\right)}{\partial x}+\frac{\partial\left(-\rho \overline{u^{\prime} v^{\prime}}\right)}{\partial y}+\frac{\partial\left(-\rho \overline{u^{\prime} w^{\prime}}\right)}{\partial z}\right]
\end{aligned}
$$




$$
\begin{aligned}
\frac{\partial V}{\partial t}+\operatorname{div}(V \mathbf{U})= & -\frac{1}{\rho} \frac{\partial P}{\partial y}+\nu \operatorname{div}(\operatorname{grad})(\mathrm{V}) \\
& +\frac{1}{\rho}\left[\frac{\partial\left(-\rho \overline{u^{\prime} v^{\prime}}\right)}{\partial x}+\frac{\partial\left(-\rho \overline{v^{\prime 2}}\right)}{\partial y}+\frac{\partial\left(-\rho \overline{v^{\prime} w^{\prime}}\right)}{\partial z}\right] \\
\frac{\partial W}{\partial t}+\operatorname{div}(W \mathbf{W})= & -\frac{1}{\rho} \frac{\partial P}{\partial z}+\nu \operatorname{div}(\operatorname{grad})(\mathrm{W}) \\
& +\frac{1}{\rho}\left[\frac{\partial\left(-\rho \overline{u^{\prime} v^{\prime}}\right)}{\partial x}+\frac{\partial\left(-\rho \overline{v^{\prime} w^{\prime}}\right)}{\partial y}+\frac{\partial\left(-\rho \overline{w^{\prime 2}}\right)}{\partial z}\right]
\end{aligned}
$$

The additional six terms are the derivatives of the Reynolds stresses, three normal stresses,

$$
-\rho \overline{u^{\prime 2}}, \quad-\rho \overline{v^{\prime 2}}, \quad-\rho \overline{w^{\prime 2}}
$$

and three shear stresses,

$$
-\rho \overline{u^{\prime} v^{\prime}}, \quad-\rho \overline{u^{\prime} w^{\prime}}, \quad-\rho \overline{v^{\prime} w^{\prime}}
$$

The compressible form of the above equations can be obtained in [50]; the above form is sufficient in demonstrating how the six Reynolds stress derivatives add six extra unknowns. The Boussinesq approach couples the Reynolds stresses to the gradient of the average velocities by an eddy viscosity, $\mu_{t}$ [51]:

$$
-\rho u_{i}^{\prime} u_{j}^{\prime}=\mu_{t}\left(\frac{\partial u_{i}}{\partial x_{j}}+\frac{\partial u_{j}}{\partial x_{i}}\right)-\frac{2}{3}\left(\rho k+\mu_{t} \frac{\partial u_{i}}{\partial x_{i}}\right) \partial_{i j}
$$

where $k$ is the average kinetic energy of the turbulence. The eddy viscosity, $\mu_{t}$, has the same dimensions as the dynamic viscosity, $\mu$, but varies depending on the velocity field. Substituting this for the Reynolds stresses allows the RANSE to be solved numerically. Various turbulence models exist for this purpose, each with its own method.

\subsubsection{Turbulence modelling}

The appearance of turbulence manifests itself as random fluctuations of the measured velocity component about a mean value. All other flow variables (pressure, temperature, density) will also exhibit this additional time-dependent behaviour [50]. This happens when the flow reaches a certain Reynolds numbers (Re) and transition from laminar to turbulent flow occurs. 
For internal flows in pipes this value is generally taken as $\mathrm{Re}=4000$, with transition from laminar to turbulent flow being where $2300<\operatorname{Re}<4000$. Here Re is calculated using the pipe diameter. Once the flow is fully turbulent, flow modelling and prediction becomes complicated, and different methods are required to deal with these types of flow. Various methods have been developed to approximate turbulence.

Turbulence models can be algebraic ( 0 equation models) or based on one or more differential equations (1- or 2-equation models), with the latter being more time consuming, but also more accurate. The algebraic models compute the eddy viscosity $\left(\mu_{t}\right)$ directly, while the 1or 2-equation models have to solve extra differential equations [50].

The most widely used turbulence model for in-cylinder engine flows is the $k-\epsilon$ model [18]. The realizable $k-\epsilon$ model is often used in practice for in-cylinder engine flows [52]. For this model, the eddy viscosity is expressed as a function of the local turbulent kinetic energy, $k$, and its dissipation rate, $\epsilon$ :

$$
\mu_{t}=C_{\mu} \rho \frac{k^{2}}{\epsilon}
$$

The empirical constant $\mathrm{C}_{\mu}$ is generally equal to $0.09 ; k$ is expressed as $\frac{\mathbf{u} \cdot \mathbf{u}}{2}$.

\section{Near wall treatment}

The velocity and temperature boundary layers occurring in engine cylinder application are generally too thin to be resolved explicitly with a suitably sized mesh. These boundary layers are however important as they determine the wall shear and heat flux. Sub-models known as wall functions can be used to connect the wall shear stresses, heat fluxes, wall temperatures, etc. to the conditions at the outer edge of the boundary layer. This eliminates the need to have a grid point in this layer. More detailed explanations of these sub-models can be found in [18, 50, 51].

The $k-\epsilon$ model cannot be applied at a wall because it assumes high local Re. The fluid is, however, stationary at the wall. In order to enforce a no-slip condition directly at the wall, a wall function coupled to the standard $k-\epsilon$ model must be introduced. The wall function, $y^{+}$, is a non-dimensional wall distance which is empirically determined for $2 \mathrm{D}$ flows [53] as:

$$
y^{+} \equiv \frac{\rho \mu_{\tau} y}{\mu}
$$

Where $y$ is the co-ordinate distance from the wall to the centre of the first cell adjacent the wall. The FLUENT ${ }^{T M}$ documentation [49] states that the centres of the cells closest to the 
wall should lie within a distance where $30<y^{+}<300$ and that it is very important that the cells adjacent the wall are not placed within the buffer layer: $y^{+}=5-30$. The buffer layer is shown in Figure 3.4 .

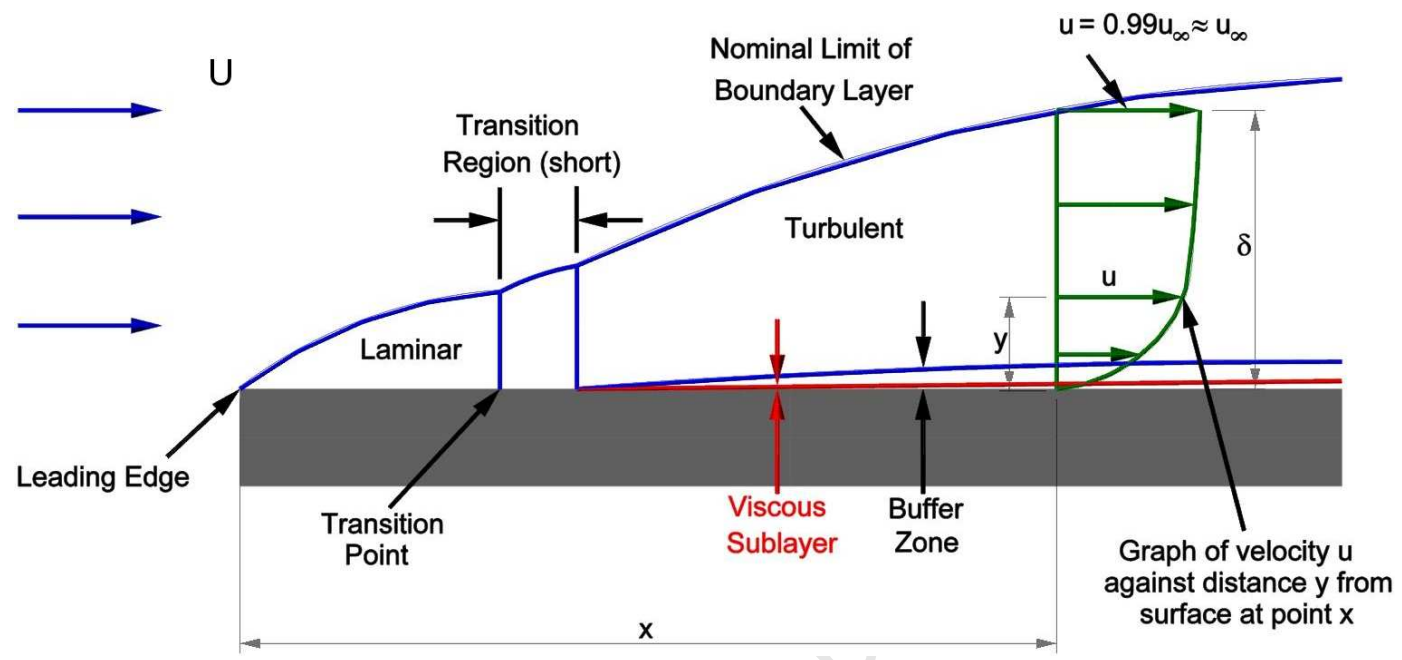

Figure 3.4: The boundary layer that forms on a flat plate, with the buffer zone indicated [54]

\subsubsection{Discretization scheme and pressure-velocity coupling}

The governing equations are converted from their differential form to an algebraic form that can be solved numerically by discretization of these equations over the entire computational grid. The discretisation scheme chosen determines how property values stored at cell centres are interpolated to give the property values at cell faces [55]. A few of the most generally used schemes are discussed briefly.

The simplest scheme is the first order upwind scheme which takes the direction of the flow into account by making the boundary value equal to the value at the node in the CV upstream of it. This becomes problematic when the property values of the flow is not normal to the CV boundary, leading to what is known as numerical diffusion. Numerical diffusion can be decreased or eliminated by using more nodes upstream to determine the property values at the CV in question. This is known as a higher order upwind approach. Here the property gradient is calculated in the CV upstream, and the property value upstream of this is used to calculate the boundary value by extrapolation. Second, third and fourth order upwind schemes can be used. The central differencing scheme which linearly interpolates between the nodes of the adjacent CVs to determine the property value at the boundary between CVs does not take into account the flow directionality and is seldom used in flows where advection and diffusion both play important roles [55]. 
The Quadratic Upstream Interpolation for Convective Kinetics (QUICK) discretisation scheme uses an upstream-weighted quadratic interpolation to solve for the boundary values. This scheme can minimise numerical diffusion and, although it can give very accurate solutions when used correctly, FLUENT ${ }^{T M}$ only allows for the QUICK scheme to be used on hexahedral meshes where unique upstream and downstream CVs can be identified. This is generally not possible when modelling engine flows where the geometry does not lend itself to complete hexahedral meshes [55]. The third-order Monotone Upstream-centred Scheme for Conservation Laws (MUSCL) blends a central-differencing scheme and a second-order upwind scheme and, unlike QUICK, is applicable to arbitrary meshes. This scheme can be particularly superior to second-order upwind schemes when used with complex 3D flows [56].

The velocity components for a given pressure are determined by the scalar equations derived from the conservation of momentum vector equations. These velocities do not always satisfy the continuity equation, and hence a pressure correction equation, derived from the continuity equation, is introduced. Most commonly, SIMPLE and Pressure Implicit with Splitting of Operators (PISO) methods for such velocity coupling are used [53. Both the SIMPLE and PISO algorithms are based on a guess-and-correct iterative method for calculating the pressure and velocity. When other scalars are coupled to the momentum equation, the calculation needs to be done sequentially. The sequence followed by CFD codes for the SIMPLE algorithm is shown in Figure 3.5 [57]. 


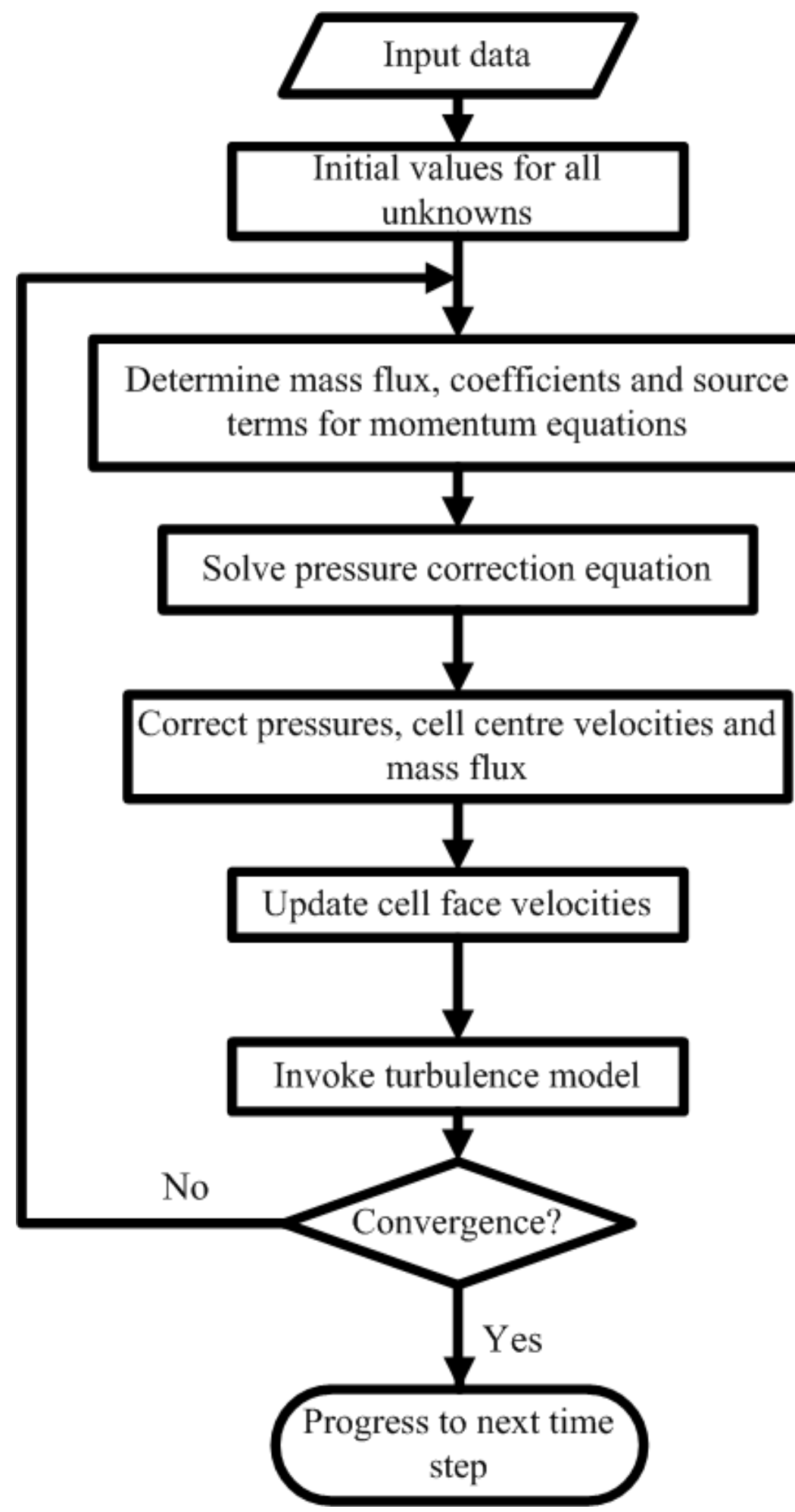

Figure 3.5: Flow chart for SIMPLE algorithm adapted from [57] 
The PISO algorithm is similar, however uses several corrector steps compared to SIMPLE. This results in PISO are more stable, but less efficient. The PISO procedure is carried out at each time level to calculate the velocity and pressure fields [58].

\subsubsection{Solution convergence: spacial and temporal discretization considera- tion}

The size and the spacing of the cells in the mesh, as well as the temporal time-steps, typically have a dramatic effect on solution accuracy.

The solution grid should be sufficiently fine so that discretization approximations become small. Generally, solutions should be run on two or more grids, one coarse and one fine. If the difference between the tow solutions are small enough to be considered negligible, then the courser grid can be considered to be sufficiently fine for discretization errors to be insignificant. This is often referred to as grid independence [59]. The recommended method for discritization error estimation is the Richardson Extrapolation (RE) method [60, 61]. This method has many shortfalls, one of which is that the approach is generally only successful when used with sufficiently fie grids [59]. An acceptable and recommended method that has been evaluated over several hundred CFD cases can be found in 62 .

For unsteady flows, the mesh size and spacing can impact on the choice of solution timestep. The dimensionless Courant number is often used to determine suitable time-step sizes. For a flow velocity, $u$, mesh size $\Delta x$ and time-step $\Delta t$, the Courant number, $C$, is given by:

$$
C=\frac{u \Delta t}{\Delta x}
$$

The restriction that the $C$ be less than 1 is often applied [59]. This can however be very limiting, since refining the grid will result in smaller time-step requirements.

Spacial and temporal resolution should be considered carefully when planning any CFD solution. These guidelines should be considered within the context of each problem, the restriction on time and computational resource, and the level of accuracy required.

\subsection{Numerical modelling of the discrete phase fluid flow}

Droplets are introduced into the calculated flow field with a certain size, velocity, mass and temperature. The trajectory equations, and any other equations describing heat or mass transfer to and from the particles, are solved by stepwise integration over discrete time steps. This method of tracking droplets is known as a Lagrangian approach and differs to the 
treatment of the continuous phase which is solved in an Eulerian framework. Coupling with the continuous phase is achieved via source terms in the Navier-Stokes equations [33, 63].

\subsubsection{Droplet particle trajectory and position}

Newton's second law of motion:

$$
\begin{array}{r}
F=m a \\
a=F^{\prime}
\end{array}
$$

where $F^{\prime}$ is the force per unit particle mass.

The main forces that act on the particle are the drag, opposite to the droplet's direction of motion, and gravity. The droplet's buoyancy is included in the gravitational force term which can be expressed as:

$$
\begin{gathered}
F_{g}=m_{d} g-V_{d} \rho_{g} g=m_{d} g-\frac{m_{d}}{\rho_{d}} \rho_{g} g=m_{d} g\left(1-\frac{\rho_{g}}{\rho_{d}}\right) \\
=m_{d} g \frac{\rho_{d}-\rho_{g}}{\rho_{d}} \\
F_{g}^{\prime}=g \frac{\rho_{d}-\rho_{g}}{\rho_{d}}
\end{gathered}
$$

Equation 3.20 is the expressed as force per unit mass. The drag force can also be expressed as force per unit droplet mass:

$$
\begin{aligned}
F_{D}=\frac{1}{2} \rho C_{D}\left(v-v_{d}\right)^{2} A_{d} & =\frac{1}{2} \rho C_{D}\left(v-v_{d}\right)^{2} \frac{3 V_{d}}{r_{d}} \\
& =\frac{1}{2} \rho C_{D}\left(v-v_{d}\right)^{2} \frac{3 m_{d}}{\rho_{d} r_{d}} \\
F_{D}^{\prime}=\frac{1}{2} \rho C_{D}\left(v-v_{d}\right)^{2} \frac{3}{\rho_{d} r_{d}} &
\end{aligned}
$$

This can now be written as an ordinary differential equation [33]:

$$
\frac{d u_{d}}{d t}=F_{D}^{\prime}+\frac{g\left(\rho_{d}-\rho\right)}{\rho_{d}}+F_{x}^{\prime}
$$

$F_{x}^{\prime}$ is an additional acceleration (force per unit mass) term that can be used to include other forces that may come into play. Equation (3.24) is integrated over time to yield the velocity of the particle at each point along its trajectory. The trajectory itself is predicted by:

$$
\frac{d x}{d t}=u_{d}
$$

Equations (3.24) and (3.25) are a set of coupled ordinary differential equations (ODEs) which can be solved using either analytical integration or numerical methods [33]. 


\subsubsection{Heat transfer to and from the droplet particles}

A modelling parameter, $T_{v a p}$, is used to indicate the onset of evaporation. This value must be specified together with other material properties for the liquid phase. It has no significant meaning other than controlling the onset of evaporation. When $T_{d}<T_{v a p}$, Equation (2.4) is applied. This equation is integrated to obtain the particle temperature at the next time value, yielding:

$$
T_{d}\left(t_{n+1}\right)=\alpha_{d}+\left[T_{d}\left(t_{n}\right)-\alpha_{d}\right] e^{-\beta_{d} \Delta t}
$$

Below $T_{\text {vap }}$ only sensible heat transfer occurs. This implies that all the heat gained by the droplet is used to increase its temperature. This is better known as the sensible heat transfer to the droplet. The heat lost or gained by the droplet appears as a source or sink in the subsequent calculations of the continuous phase 33].

\subsubsection{Mass transfer to / from droplet particle}

When the droplet temperature surpasses $T_{\text {vap }}$, the liquid starts to evaporate and now some of the heat gained by the droplet is used to evaporate the liquid. This is known as latent heat transfer. A certain amount of latent heat is required to evaporate a certain mass of fuel liquid. The mass evaporated during a time step is calculated first. Next, the latent heat required to evaporate this mass is determined. The mass of fuel evaporated is calculated from Equation (2.12) which results in:

$$
m_{\text {evaporated }}=N_{i} A_{d} M_{w, i} \Delta t
$$

The definition of the vapour pressure, $p_{s a t}$, is critical, as it is used to obtain the driving force for the evaporation process. Accurate vapour pressure values for temperatures over the entire range of possible droplet temperature must be provided. $M_{w, i}$ is the molecular weight of species $i$. Note that the rate of vaporization, $N_{i}$, is assumed to be positive. If it becomes negative, then the droplet is treated as inert $\left(N_{i}=0\right)$; no mass transfer will be calculated and any heating will either raise or lower the droplet temperature. The mass of the droplet is updated according to [33]:

$$
m_{d, n+1}=m_{d, n}-N_{i} A_{d} M_{w, i} \Delta t
$$

The mass evaporated appears as a source term in the fuel vapour species transport equation.

\subsubsection{Overall heat transfer to droplet}

The droplet temperature is updated according to the heat balance that relates the sensible heat change in the droplet to the convective and latent heat transfer between the droplet and 
the continuous phase. The same is done for the continuous phase via source or sink terms in the Navier-Stokes equations for each fluid volume.

$$
m_{d} c_{d} \frac{d T_{d}}{d t}=h A_{d}\left(T_{\infty}-T_{d}\right)+\frac{d m_{d}}{d t} \mathrm{LHV}
$$

where:

$$
\begin{aligned}
& \text { LHV - latent heat of vaporisation }\left(\mathrm{J} \mathrm{kg}^{-1}\right) \\
& \frac{d m_{d}}{d t} \text { - rate of evaporation }\left(\mathrm{kg} \cdot \mathrm{s}^{-1}\right)
\end{aligned}
$$

Any heat transferred to or from the liquid phase appears as a source or sink in subsequent continuous phase calculations [33].

\subsection{Numerical modelling of the wall film fluid flow}

Spray-wall interaction plays an important part in mixture preparation in IC engines where the fuel is injected upstream of the inlet valve. The fuel spray impinges on the manifold walls, inlet port surfaces and also the intake valve. It can form a film on these surfaces or splash and subsequently evaporate. All the fuel does not evaporate and several cycles worth of fuel could remain in the intake tract due to this film formation 33 .

The wall film model in FLUENT ${ }^{T M}$ allows a liquid droplet to impinge on a boundary and form a thin film. Only single component droplets can be used with the wall film model. The schematic shown in Figure 3.6 shows the basic mechanisms which need to be considered for the wall-film model.

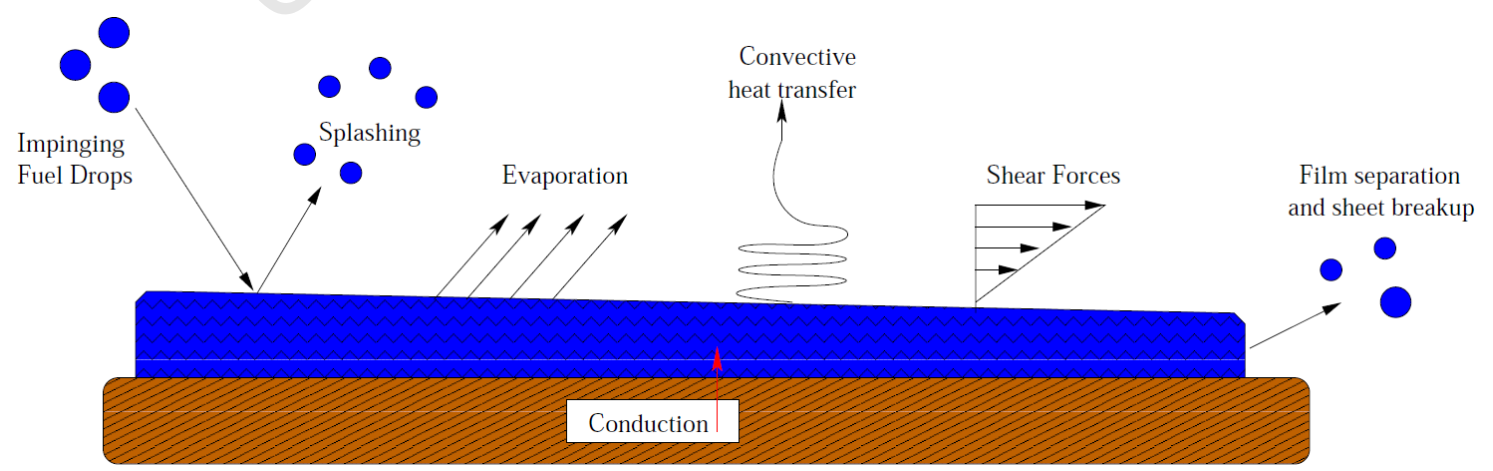

Figure 3.6: Wall film mechanisms involved when using the wall film model in FLUENT ${ }^{\mathrm{TM}}$ [33]

This section discusses the model's basic working briefly. More detailed information can be found in [33]. 
The interaction between the film and the spray is based on the impact energy (defined in [33]) and the boiling temperature of the liquid. This is illustrated in Figure 3.7.

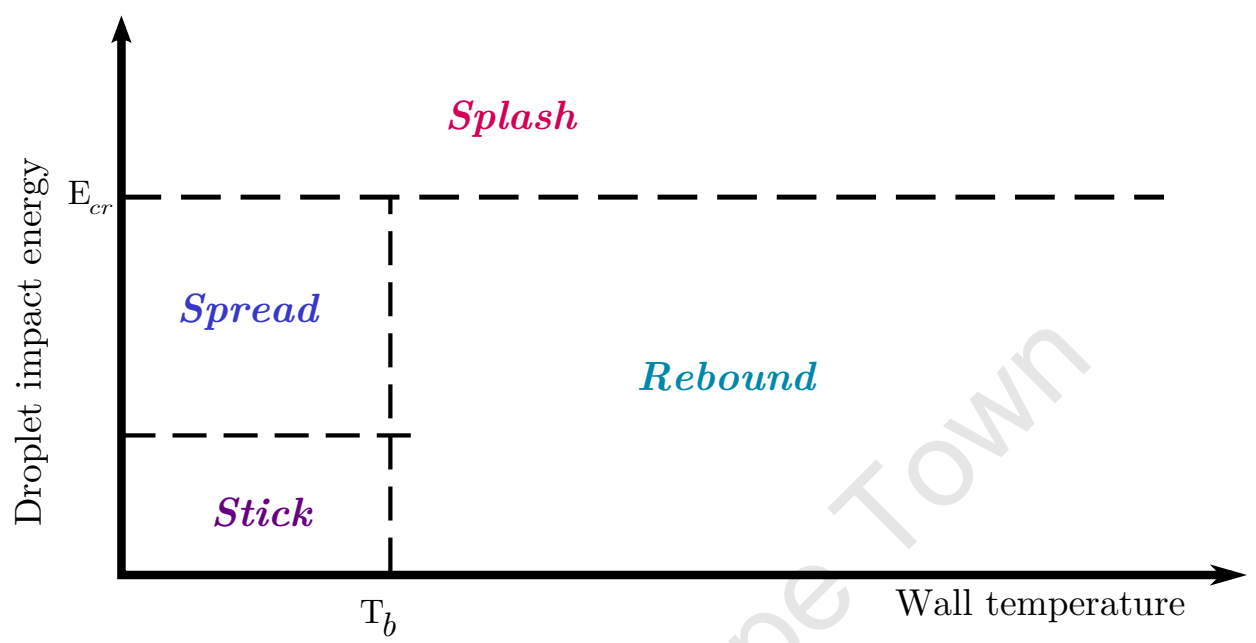

Figure 3.7: Wall interaction criteria. Figure adapted from [33]

If the wall temperature is below the boiling temperature of the liquid, the droplet will either stick, spread or splash, depending on its impact energy; above the boiling temperature of the liquid, the droplet can splash or rebound, also depending on its impact energy. Above a critical impact energy, $E_{c r}$, the droplets will always splash.

The equations governing the wall-film particles are derived from the conservation equations for momentum, mass and energy for individual parcels in the wall-film. Since the standard wall-film model used by FLUENT ${ }^{T M}$ was implemented in the current research, the reader is referred to [33] for detailed derivations of the wall-film equations.

\subsection{Coupling between fluid and liquid phase}

As already mentioned, coupling with the continuous phase occurs via source terms in the Navier-Stokes equations. Source terms are calculated for momentum, energy and mass. 


\section{Numerical approach}

Firstly, a simple pipe section was modelled to represent the intake manifold. This was followed by a $2 D$ planar model of the CFR engine intake and cylinder geometry where the port geometry and dynamic motion of the engine components were included. Finally this was translated into a 3D model. This chapter gives a brief overview of the initial $2 D$ model development. The 3D model is then discussed in more detail. Details regarding the geometry and grid generation, the dynamic model setup, thermodynamic models, BCs, injections and material properties are also discussed.

\subsection{Initial models used in the development phase}

Two 2D models were used to validate the modelling methodology that would be used for the final 3D engine model. This Section also includes the 3D model calibration of the heater element temperatures for the MON tests.

The first of the 2D models was developed based on the work in [7, 8] and consisted of a simple 2D pipe geometry based on the CFR engine manifold. The geometry and boundary types are shown in Figure 4.1.

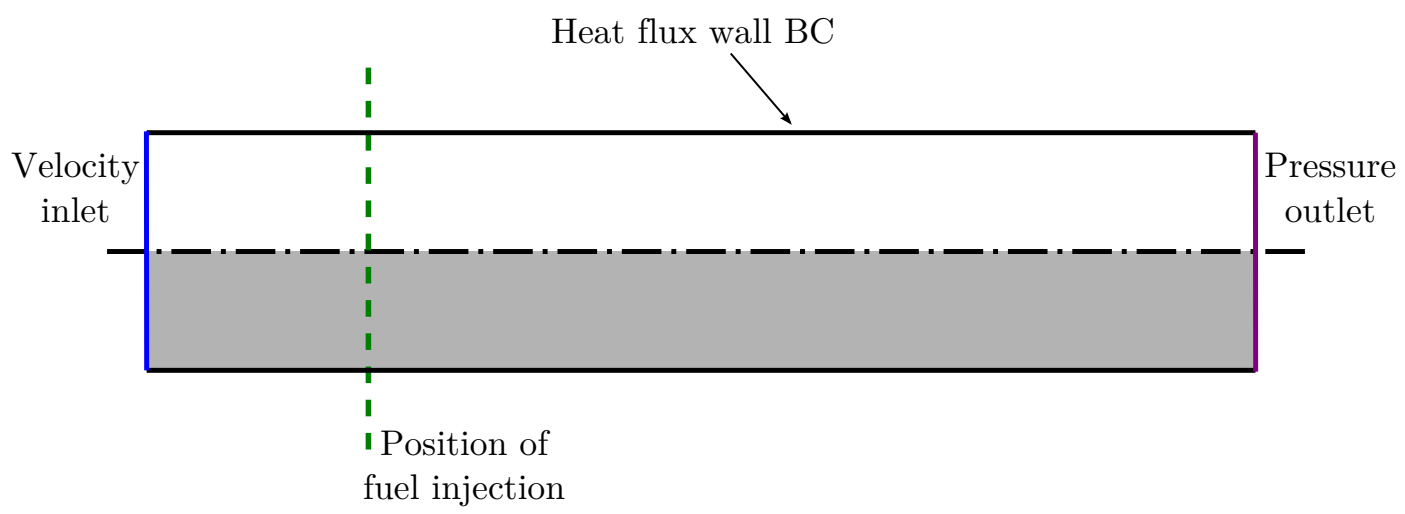

Figure 4.1: Geometry and boundary conditions for the 2D manifold model

An axisymmetric approach was used whereby only one half of the longitudinal cross section 
was modelled; the axis of symmetry being the centreline of the pipe. The cross section modelled was a constant $16 \mathrm{~mm}$ in height (the radius of the manifold) and $130 \mathrm{~mm}$ in length. The fuel injection point was set at $30 \mathrm{~mm}$ downstream from the air inlet, with the pressure outlet $130 \mathrm{~mm}$ downstream from the inlet. A pressure outlet boundary condition was applied at the end of the pipe.

Air entered the manifold via the velocity inlet at $5 \mathrm{~m} \cdot \mathrm{s}^{-1}, 325 \mathrm{~K}$ and $101.3 \mathrm{kPa}$. A constant heat flux of $100 \mathrm{~W} \cdot \mathrm{m}^{-1}$ was applied to the manifold walls. The fuel droplets were injected with initial diameters and temperatures of $50 \mu \mathrm{m}$ and $295 \mathrm{~K}$ respectively, and with zero initial velocity. The steady state solver was used with first order discretisation and the realizable $\mathrm{k}-\epsilon$ turbulence model.

The main aims of this simple pipe model were to:

1. test the iso-octane material property models implemented by comparing the results with those found in [7, 8];

2. gain an understanding of the DPM and wall film models used in FLUENT ${ }^{T M}$.

Once this was achieved, a 2D planar model which incorporated the port, valve and combustion chamber, as shown in Figure 4.2, was created.

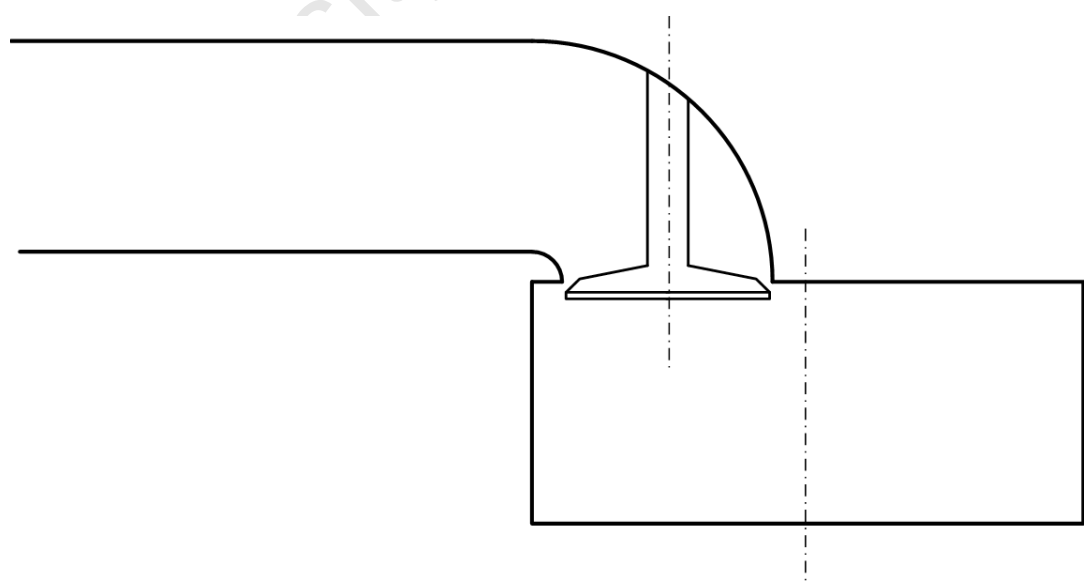

Figure 4.2: Geometry used for the 2D planar model

All dimensions were based on that of the CFR engine which can be found in Chapter 2. The main aims using the 2D planar model were to:

1. implement the dynamic motion of the piston and valve;

2. implement in-cylinder events such as valve opening and closure; 
3. implement dynamic mesh layering;

4. develop a UDF to inject fuel droplets with a mass flow rate that would result in a stoichiometric AFR within the cylinder.

The stoichiometric AFR is 15.18 for iso-octane-air, and 13.5 for toluene-air. The fuel equivalence ratio, $\gamma$, is often used to express the state of the air-fuel mixture given by:

$$
\gamma=\frac{A F R_{\text {actual }}}{A F R_{\text {stoichiometric }}}
$$

The value of $\gamma$ is calculated taking into account gaseous components only and indicates a rich mixture when $\gamma<1$ and a lean mixture when $\gamma>1$.

Such a planar model was, however, not able to account for the in-cylinder swirl and gas dynamics, nor the unique inlet valve geometry, which are inherently 3D. The heating manifold which is used during the MON tests could also only be included in a 3D model. Once the above mentioned objectives were satisfied, a more representative, 3D model could be developed, based on the methodology followed for the $2 \mathrm{D}$ models.

\subsection{D Geometry}

The final geometry and mesh were created using the commercial pre-processor GAMBIT ${ }^{T M}$. The methodology applied in developing the 2D models was carried over to a full 3D model of the CFR engine model. Both the RON and the MON models consisted of an intake manifold, intake port, inlet valve and combustion chamber. For the MON case, an extra heating manifold section was inserted between the intake manifold and intake port. The MON setup is shown in Figure 4.3a, 


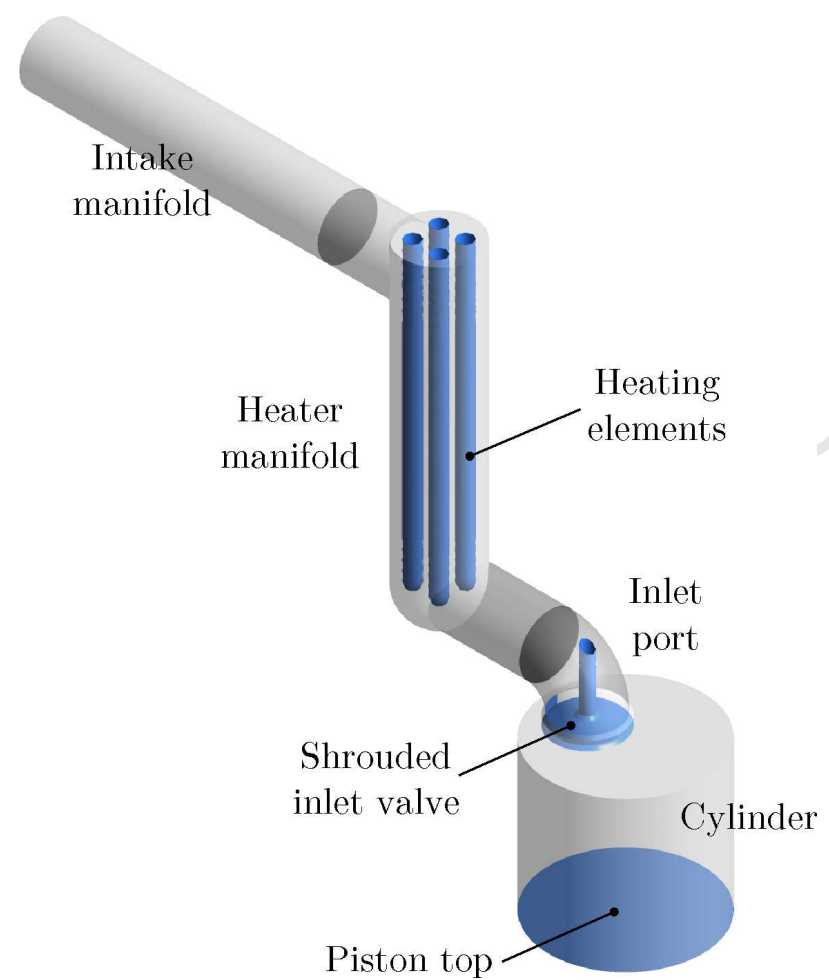

(a) $M O N$

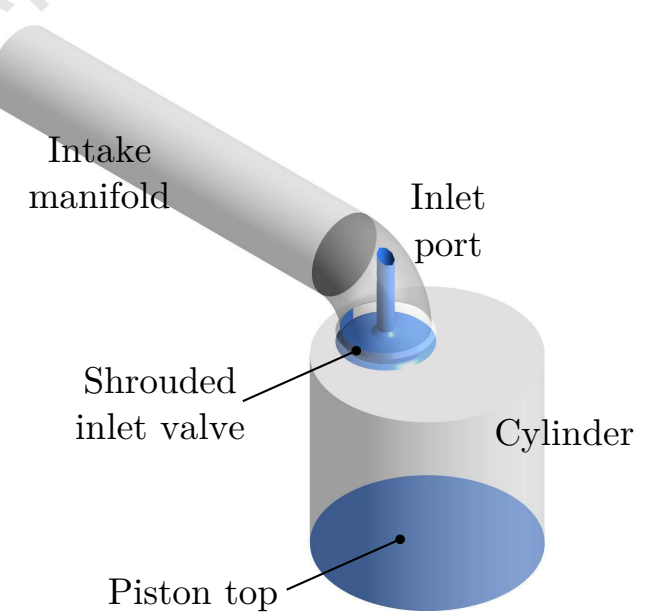

(b) $R O N$

Figure 4.3: $C F D$ model geometries used for $3 D$ simulations 
The RON geometry was similar, however did not have a heater manifold connecting the intake manifold to the inlet port. The simpler RON setup is shown in Figure 4.3b,

The special inlet valve geometry could be included in the 3D geometries and is shown close up in Figure 4.4.
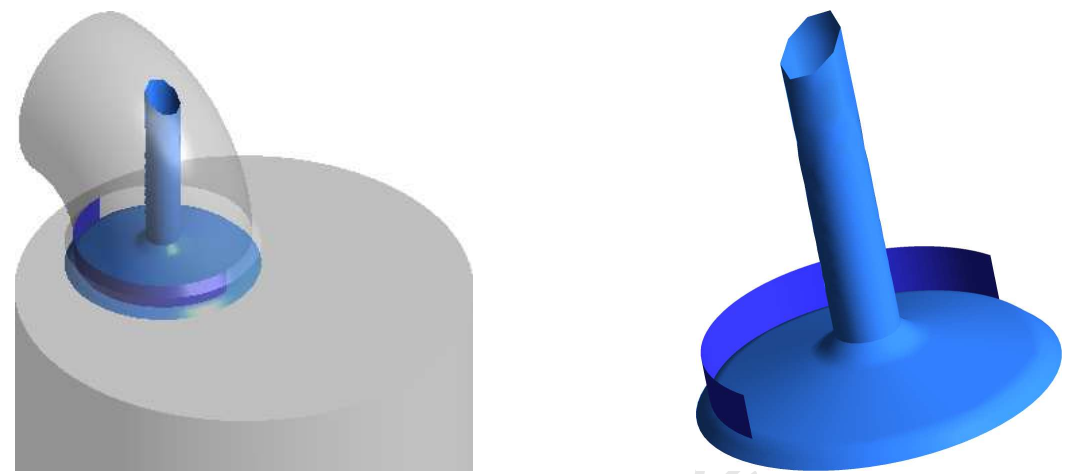

Figure 4.4: Inlet port with shrouded inlet valve

The geometry of the valve shroud was simplified to be a half moon wall which blocked off one half of the valve curtain area. The exhaust valve, port and manifold were not included in order to decrease the complexity of the geometry. The exhaust process was thus approximated and this is covered further on in this chapter.

\subsection{Computational grid}

The computational grids were created in $\mathrm{GAMBIT}^{T M}$. Grids were developed for the 2D planar geometry, and both the RON and MON 3D geometries. Meshing regions refer to regions of structured, unstructured, hexahedral or tetrahedral cells. It also refers to dynamic mesh regions such as remeshing or layering zones. The computational grid used for the MON setup is shown in Figure 4.5, with the mesh element types used in each region clearly indicated. 


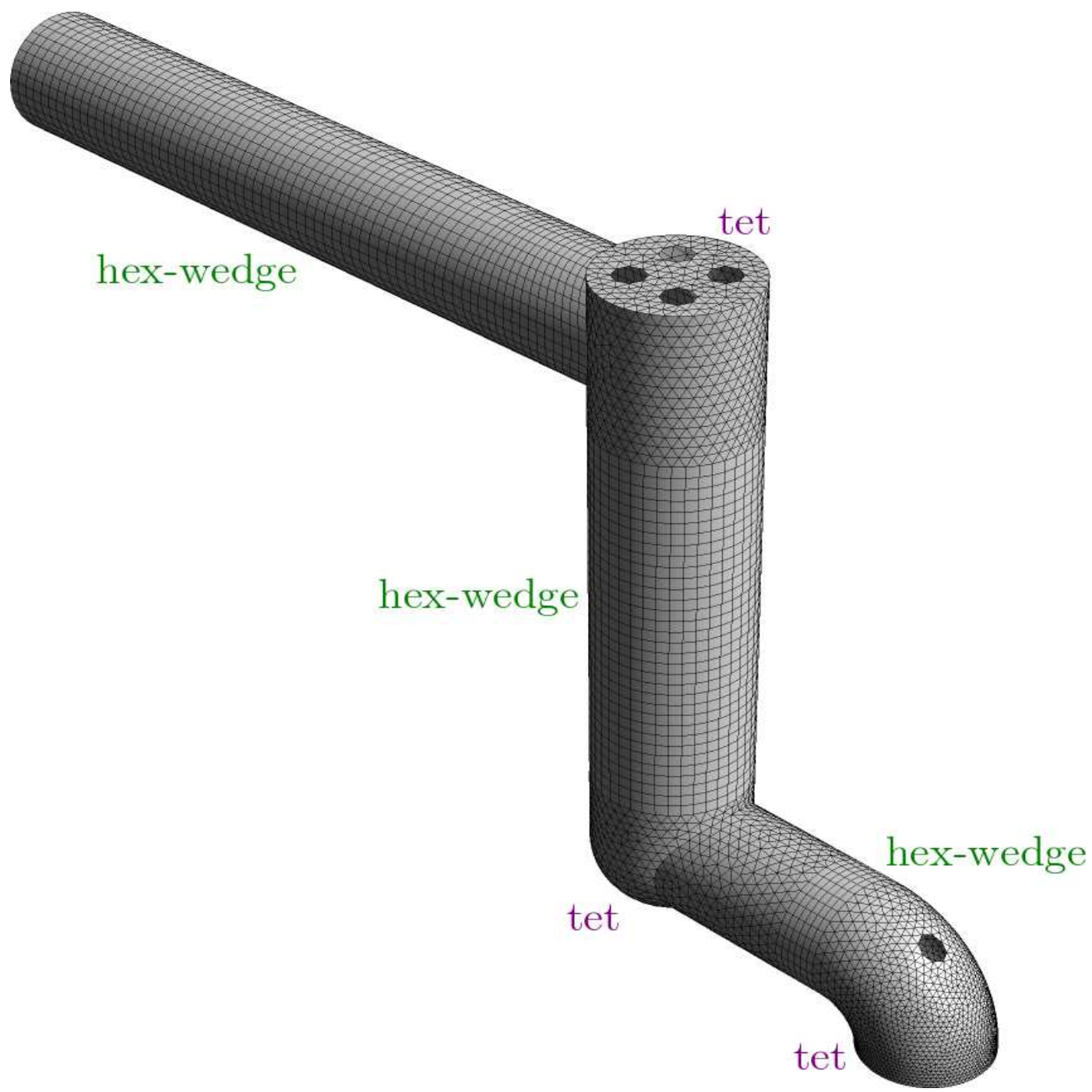

Figure 4.5: Computation grid used for the MON intake manifold, heating manifold and intake port sections 
The bends and protruding internal structures, such as the heating elements and inlet valve, made it challenging to have a uniformly structured grid in these regions. It was thus necessary to have hex-wedge elements where the geometry was simple, and tetrahedral elements in the manifold and port regions. For the RON setup a similar grid was used, except the heater manifold section was removed. This is shown in Figure 4.6.

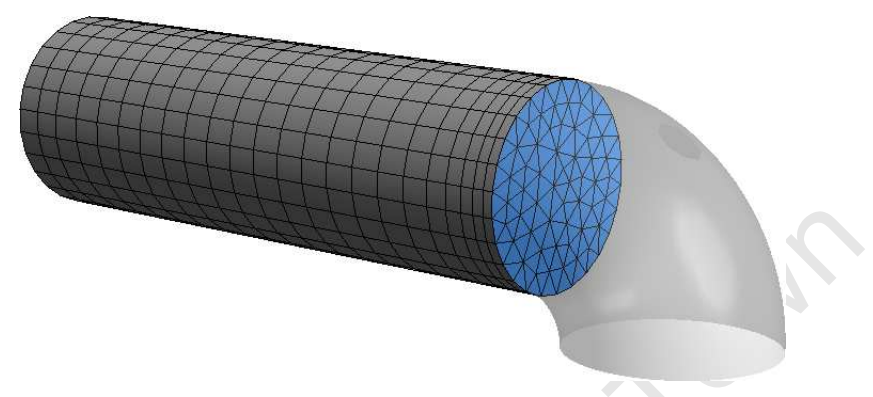

Figure 4.6: Computational grid used for the RON intake manifold section

The internal unstructured mesh can also be seen. The in-cylinder grid consisted of a semistructured mesh with only hex-wedge grid elements. The cylinder top and piston face meshes are shown in Figure 4.7 .

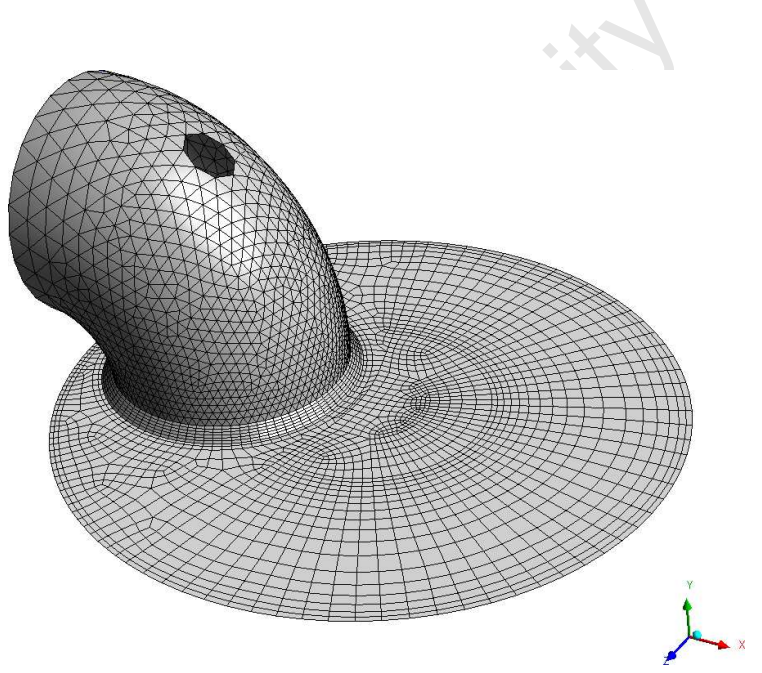

(a) Cylinder top

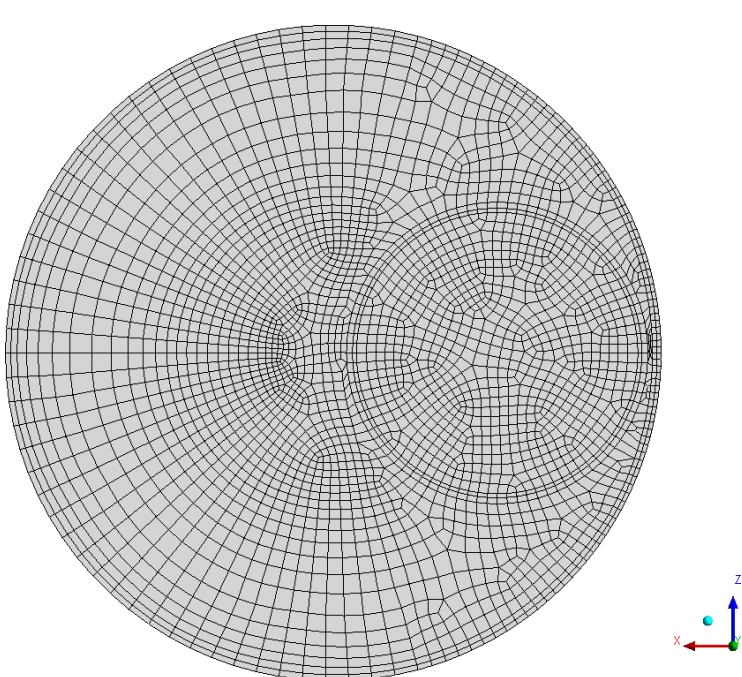

(b) Piston top

Figure 4.7: Face meshes used for the combustion chamber for both RON and MON models

Dynamic mesh layering was implemented with the moving valve and piston boundaries. Mesh layering regions were used to model the moving valve and piston. The valve required 2 layering regions, one above and one below the valve. The piston only required one mesh layering region, as elements were only ever removed or added within the cylinder space above the piston top 
wall. The mesh layering which was applied to the inlet port region is shown in Figure 4.8 for the 2D planar case. The same method of mesh layering was applied in the 3D model.

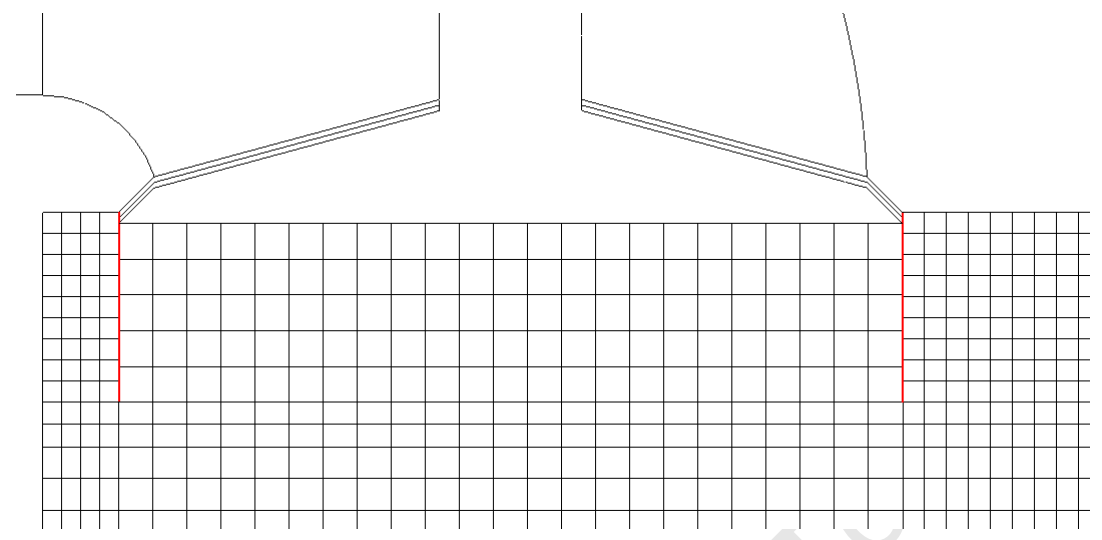

(a) $I V C$

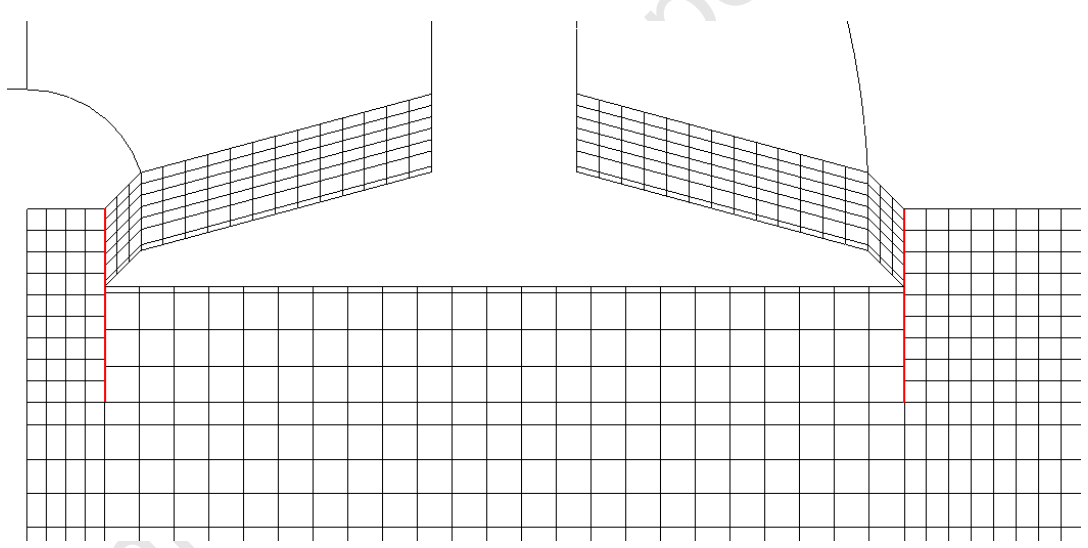

(b) IVO

Figure 4.8: Dynamic mesh layering above and below the inlet valve. The grid shown was for the 2D planar model geometry.

During the opening of the valve, grid elements are added above the valve. The existing layer of grid elements stretches until it reaches a pre-set height, where after the row of elements splits adding a new row of elements which then stretch as the valve opens further. The opposite happens underneath the valve as it opens. The row of elements underneath the valve effectively shrinks until it disappears completely. As the valve closes, the same happens in reverse: elements are added below the valve and removed above it. Since the grid in the surrounding combustion chamber does not change, mesh interfaces (shown in red on Figure 4.8 were required between the layering regions and the surrounding combustion chambers. An interface consists of two surfaces representing the same area but with independent face meshes. More details regarding dynamic meshing can be found in [64]. 
Grid independence studies were not conducted on the mesh due to time constraints. Instead, it was ensured that fine mesh elements were used in the areas where high gradients were expected, such as through the valve. Given the various simplifying assumptions that was made to reduce the problem such that useful comparative information could be extracted in a practical time-frame, it was ensured that the grid be near-identical for all the simulations, even though no grid independence studies were done.

\subsection{Dynamic model setup}

FLUENT $^{T M}$ 's in-cylinder model [64] was used to move the piston and valve boundaries at the correct speed based on the engine geometrical parameters shown diagrammatically in Figure 4.9,

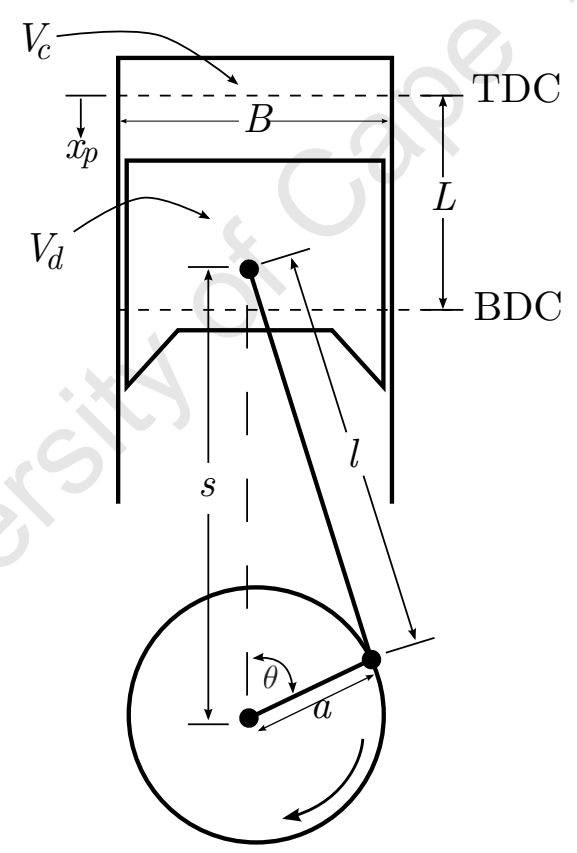

Figure 4.9: General engine geometry and parameters. Schematic adapted from [65].

Following the labelling in Figure 4.9, the position of the piston top can be expressed as [65]:

$$
x_{p}=l+a-s
$$

where:

$x_{p}$ - piston displacement measured from TDC

$l$ - connecting rod length

a - crank radius

$s \quad-$ stroke 
The specific inputs to the in-cylinder model for the CFR engine are given in Table 4.1.

Table 4.1: Engine parameters for in-cylinder model

\begin{tabular}{|c|c|c|c|}
\hline & Unit & $\mathrm{RON}$ & MON \\
\hline Crank shaft speed & {$[\mathrm{RPM}]$} & 600 & 900 \\
\hline Starting crank angle & {$\left[\mathrm{CA}^{\circ} \mathrm{ATDC}\right]$} & 0 & 0 \\
\hline Crank period for modelling ${ }^{a}$ & {$\left[\mathrm{CA}^{\circ}\right]$} & 1080 & 1440 \\
\hline Piston stroke & {$[\mathrm{mm}]$} & 114.3 & 114.3 \\
\hline Con rod length ${ }^{b}$ & {$[\mathrm{~mm}]$} & 254 & 254 \\
\hline $\begin{array}{l}{ }^{a} \text { This is the crank period used } \\
720^{\circ} \mathrm{CA} \text {. }\end{array}$ & delling and & & \\
\hline
\end{tabular}

The valve lift profile was approximated based on a generic sinusoidal wave equation adapted based on the CFR inlet valve timing:

$$
L_{v}=L_{\max }\left(\frac{1-\cos \phi}{2}\right)^{m}
$$

where $\theta$ is the instantaneous crank angle and $\phi$ is given by:

$$
\phi=2 \pi \frac{\theta-\theta_{I V O}}{\theta_{I V C}-\theta_{I V O}}
$$

The valve was assumed shut at a valve lift of approximately $1 \mathrm{~mm}$. At very small valve lifts the solution becomes computationally expensive, as very high gradients of velocity and acceleration exist here. In order to circumvent this, the valve is assumed closed before this becomes computationally too expensive. Closing the valve translates to changing the $\mathrm{BC}$ type of the valve curtain area from a 'wall' to an 'interior'. In the models developed, the valve was 'shut' at $30^{\circ} \mathrm{ABDC}$, as opposed to $34^{\circ} \mathrm{ABDC}$. Even though the valve was still open $(1 \mathrm{~mm}$ valve lift), the flow from the manifold into the cylinder was stopped at this stage by closing up the remaining valve curtain area ('interior' BC to 'wall' BC). The inclusion of the valve movement resulted in increased computational complexity as there were much higher velocity gradients at low valve lift. Back-flow into the manifold also occurred as the valve reached the near-closed position. These were expected to have greater influence when the fuel spray was introduced. Closing the valve (by deactivating the intake zone) just before it reaches IVC (i.e. when the valve lift is close, but not equal, to zero) was one way of dealing with the convergence problems that arose. This is standard practice in most in-cylinder engine flow applications [64]. 


\subsection{Solver setup}

The following models were used during the solution calculation:

1. Turbulence model.

2. Species transport model.

3. Discrete phase model (DPM).

The realizable $k-\epsilon$ turbulence model [66] with standard wall functions was used for all simulations. The default values for all turbulence modelling parameters were used as this is generally recommended [50]. The species transport model contained in FLUENT ${ }^{T M}$ [67] was incorporated. The coupled DPM model [63] was used with the DPM source set to be updated at every flow iteration. This means that the flow field calculations and droplet spray tracking was performed iteratively and that any mass, momentum or energy transfer between the two phases were updated at every flow iteration. Unsteady particle tracking was used, with the particle time step $0.1 \mu \mathrm{s}$, and injection of liquid occurring at the particle time step.

The unsteady, pressure based solver was used. The PISO pressure-velocity scheme [68] with the second order spatial discretisation scheme was used as it resulted in stable convergence compared to lower order discretisation schemes. Second-order upwind differencing was used for pressure, density and momentum, whilst first order upwind differencing was used for all remaining equations. First order upwind differencing was investigated for pressure and density, but convergence was only achieved when using second order for pressure.

Residuals were used to monitor the level of convergence of an iterative procedure. The difference of each flow property as it entered and left a CV was calculated at each iteration. The residual for a given flow property was then defined as the sum total of all these differences over the entire domain. It was then ensured that the residuals decreased towards zero during each iteration. Convergence was assumed to be reached when the residuals were sufficiently small. There is no set magnitude for these residuals. Where numerical accuracy is the major objective, residuals as small as $1 \times 10^{-6}$ would be preferred. However, $1 \times 10^{-3}$ was chosen to be sufficient as it ensured convergence at every time step. For each time step, convergence within 10 to 25 iterations was aimed for. This could be controlled to some extent by adjusting either the time step size, the mesh, or the relaxation factors.

Relaxation factors were used to damp solutions from previous iterations in order to prevent a solution from becoming unstable. The default relaxation factors were used in all calculations except for pressure and momentum. It was found that 0.5 for both pressure and momentum resulted in more stable convergence. The relaxation factor for the DPM phase was set to 1. 


\subsection{Problem setup}

Initial and boundary conditions drive the solution for any CFD simulation, and thus these have to be specified correctly as to best represent the process that is being modelled. Initial and boundary conditions, as well as fuel injection properties were specified. The injections were initialized at the plane indicated in Figure 4.10 for the MON setup and in Figure 4.11 for the RON setup.

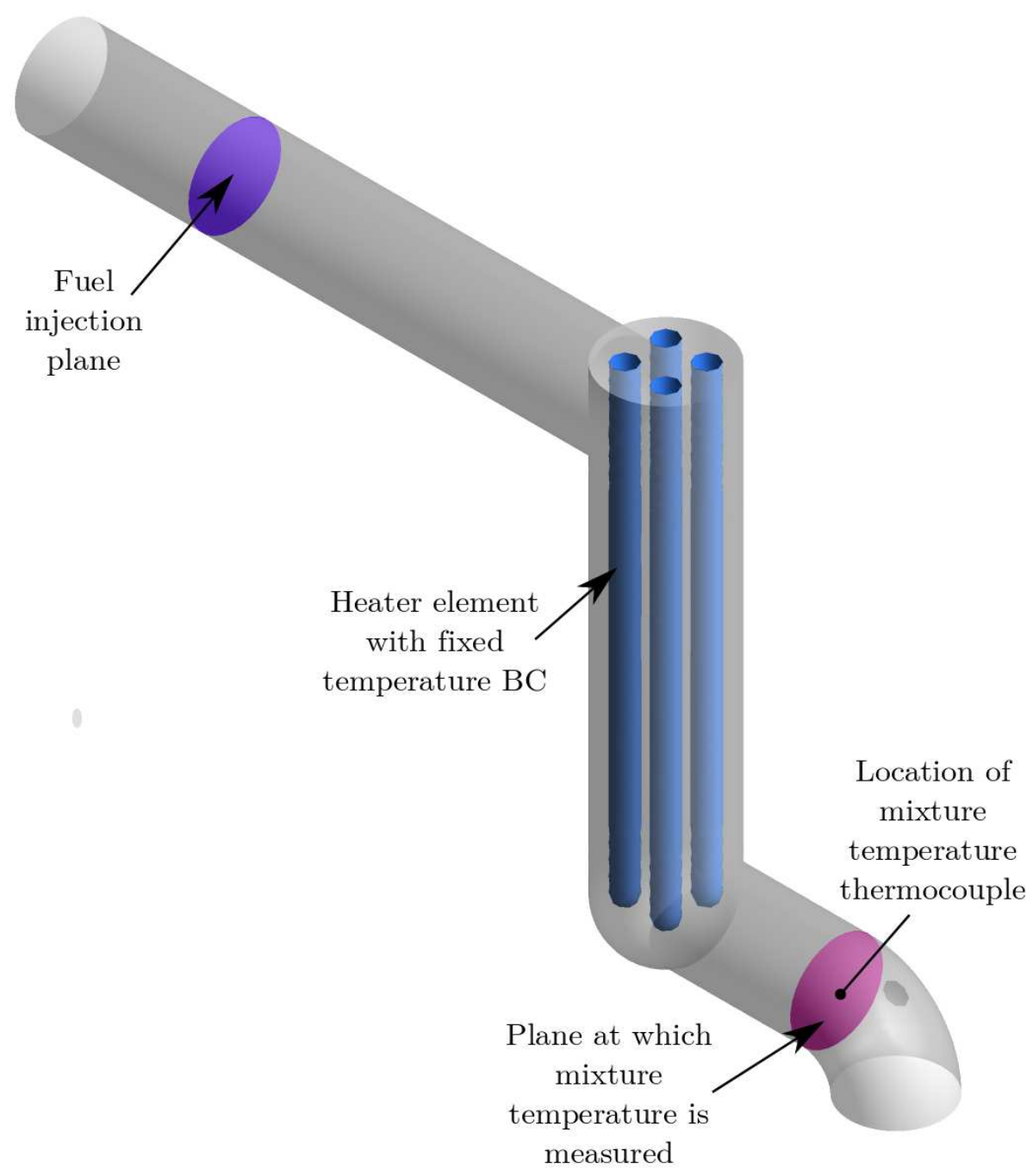

Figure 4.10: Special considerations during problem setup (MON) 


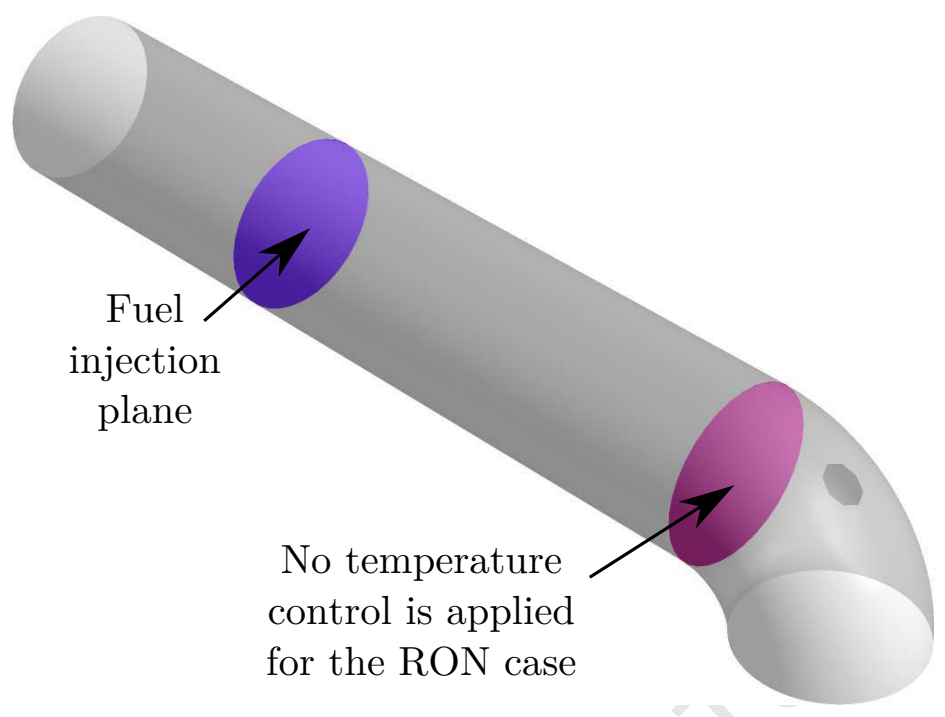

Figure 4.11: Special considerations during problem setup (RON)

The mixture temperature for the MON case was controlled by fixing the heating elements' temperature to a suitable value.

\subsubsection{Initial conditions}

The initial in-cylinder pressure and temperature was estimated based on a 0D thermodynamic CFR engine model and specified at IV0, the values being $9500 \mathrm{~Pa}$ and $875 \mathrm{~K}$ respectively. These values were taken from a 0D CFR engine model created by Perumal for use in [17]. The mass fraction of combustion products were calculated assuming stoichiometric combustion. These initial conditions are summarized in Table 4.2 .

Table 4.2: In-cylinder conditions after the exhaust stroke at IVO

\begin{tabular}{lcc}
\hline & Iso-octane & Toluene \\
\hline Temperature & \multicolumn{2}{c}{$875 \mathrm{~K}$} \\
Gauge pressure & \multicolumn{2}{c}{$-5 \mathrm{kPa}$} \\
Carbon dioxide mass fraction & 0.1918 & 0.2305 \\
Nitrogen mass fraction & 0.7199 & 0.7156 \\
Water vapour mass fraction & 0.0883 & 0.0539 \\
\hline
\end{tabular}

The mass fraction of combustion products (carbon dioxide, nitrogen and water vapour) were calculated from the stoichiometric AFR, which is 15.18 for iso-octane and 13.5 for toluene. 


\subsubsection{Boundary conditions}

All the models were developed in such a way as to mimic the CFR engine as closely as possible. The initial and boundary conditions were chosen to represent either RON or MON test conditions. A compression ratio of 7 was used for all initial $2 \mathrm{D}$ studies. For the 3D models, a CR of 7.8 was used for all studies, since this corresponds better to knocking in the CFR engine using iso-octane. A sketch indicating the different boundary sections was included previously in this chapter in Figure 4.3a,

\section{Air inlet}

The air inlet was modelled with a pressure inlet BC, where the mass flow of air is calculated based on the pressure differential between the inlet air pressure and the in-cylinder pressure. The values that were specified are given in Table 4.3 .

Table 4.3: $B C s$ at the air inlet

\begin{tabular}{lcc}
\hline & RON & MON \\
\hline Temperature & $325 \mathrm{~K}$ & $312 \mathrm{~K}$ \\
Pressure & $101.3 \mathrm{kPa}$ \\
Hydraulic diameter & $32 \mathrm{~mm}$ \\
Turbulent intensity & $10 \%$ \\
Mass fraction of oxygen & 0.21 \\
\hline
\end{tabular}

The hydraulic diameter was taken to be the diameter of the inlet manifold pipe. Since it would be expected that the air enters the intake manifold with some level of turbulence, the turbulent intensity here was approximated to be $10 \%$.

\section{Wall temperatures}

These temperatures were chosen to best represent the CFR engine and is given in Table 4.4. 
Table 4.4: Wall temperature BCs

\begin{tabular}{lcc}
\hline & RON & MON \\
\hline Intake manifold & \multicolumn{2}{c}{$300 \mathrm{~K}$} \\
Heating manifold & - & $325 \mathrm{~K}$ \\
Heating elements & - & variable \\
Inlet Port & $300 \mathrm{~K}$ & $325 \mathrm{~K}$ \\
Valve (in port) & $300 \mathrm{~K}$ & \\
Valve (in cylinder) & \multicolumn{2}{c}{$400 \mathrm{~K}$} \\
Cylinder walls & $400 \mathrm{~K}$ \\
Piston top (in cylinder) & $400 \mathrm{~K}$ \\
\hline
\end{tabular}

In both the RON and MON setup, the manifold walls are exposed to the atmosphere and could readily be touched. The temperature was thus chosen to be $6 \mathrm{~K}$ hotter than room temperature $(300 \mathrm{~K})$. For the MON setup, the heater manifold walls were assumed to be hotter than the intake manifold walls. On inspection, during running of these tests, the heating manifold was hot to the touch, but tolerable, and was chosen to be roughly $28 \mathrm{~K}$ above ambient temperature (325 K). This same temperature was applied to the inlet port for the MON case.

The heating element temperatures were found through an iterative method. This was done by choosing a temperature and then monitoring the resulting temperature at the thermocouple location as well as at the plane where it is found. These were clearly indicated in Figure 4.10. The resulting mixture temperature was time-averaged and then compared to the requirement which is $422 \mathrm{~K}$. This is discussed further in Chapter 5 .

The combustion chamber inner surfaces (cylinder walls, piston top and valve bottom) were set to $400 \mathrm{~K}$. The coolant temperature for the RON and MON tests are specified at $373 \mathrm{~K}[5]$. In a study by Marr et al. in [12], a fast response thermocouple was developed for measuring surface temperatures of aluminium components in IC engine combustion chambers. Experimental measurements using these surface thermocouples in the CFR engine indicated a wall temperatures of $423 \mathrm{~K}$. For the current study the combustion chamber wall temperature of $400 \mathrm{~K}$ was thus chosen as the average of the specified coolant temperature and the experimental measurements in [12].

\section{Operating conditions}

The operating pressure and density, as well as the gravity acceleration, were specified. The operating pressure was set to atmospheric pressure $(101.325 \mathrm{kPa})$. The location of this reference pressure was set in the manifold section, upstream from both the inlet valve and 
fuel injection plane. This location was chosen as it is in an area where the density remains near-constant, and also where the pressure variations will be the least. The operating density was set to that of air at standard temperature and pressure $\left(1.225 \mathrm{~kg} . \mathrm{m}^{-3}\right)$. The gravitational accelertaion was set to $9.81 \mathrm{~m} \cdot \mathrm{s}^{-2}$ acting parallel to the cylinder axis.

\subsubsection{Injection set-up}

For the planar model a group injection containing 10 streams was used to represent the fuel spray. The droplets were ascribed initial diameters of $50 \mu \mathrm{m}$ based on the findings in [8]. The initial droplet temperature was set to just above room temperature at $295 \mathrm{~K}$. The initial velocity of the spray was set to be zero in all directions. These parameters are summarized in Table 4.5,

Table 4.5: Summary of injection properties

\begin{tabular}{lc}
\hline Temperature & $295 \mathrm{~K}$ \\
Velocity (x, y. z) & $0,0,0$ \\
Flow rate & $\mathrm{UDF}$ \\
Diameter & $50 \mu \mathrm{m}$ \\
No. of streams & 10 \\
Cone angle & $0.5^{\circ}$ \\
\hline
\end{tabular}

The liquid fuel injection occurred during the intake stroke (IVO to IVC) with a time-dependent mass flow rate that was calculated using a UDF. The UDF is documented in Appendix A. The random walk model was used for turbulent dispersion of the droplets.

\subsubsection{Materials set-up}

The fuel-air mixture was treated as ideal gas for compression. The fuel liquid's saturation vapour pressure was specified over a range of operating temperatures based on the models discussed in Section 2.4.3.

\subsection{Modelling procedure sequence}

This section gives a brief overview of the modelling procedure that was implemented to model the intake and compression cycles, and to approximate the power and exhaust cycles.

The main aim of the research presented here was to gain insight into the dynamics and heat transfer which occur during intake and compression in the CFR engine. Modelling of 
the power and exhaust cycles would increase the computational cost and time dramatically, and would require many further simplifications and assumptions. These cycles were thus not modelled, but rather approximated using initial and boundary conditions as per Table 4.2. and disabling - or rather freezing - any in-cylinder (i.e. combustion chamber) calculations during these cycles. The manifold flow modelling was continued during the rest of the engine cycle to account for dynamic effects, pulsations and momentum during the time that the inlet valve was closed.

The intake cycle was initiated at IVO by applying the initial conditions given in Table 4.2 to the in-cylinder computational domain. The intake cycle was initiated at IVO (10 ATDC) by effectively opening the inlet valve as follows:

1. Delete existing mesh interfaces.

2. Change the BC type of the valve curtain area from a wall to an interface.

3. Redefine the meshing interfaces between the port and cylinder.

At IVC, the inlet valve was effectively closed as follows:

1. Delete existing mesh interfaces.

2. Change the $\mathrm{BC}$ type of the valve curtain area from an interface to a wall.

3. Redefine the meshing interfaces between the port and cylinder.

For the RON tests, the modelling of two intake cycles were required to allow the fuel spray travel over the entire manifold distance and into the cylinder. This increased to four intake cycles for the MON case, since the manifold volume was greater due to the extra heating manifold section. 


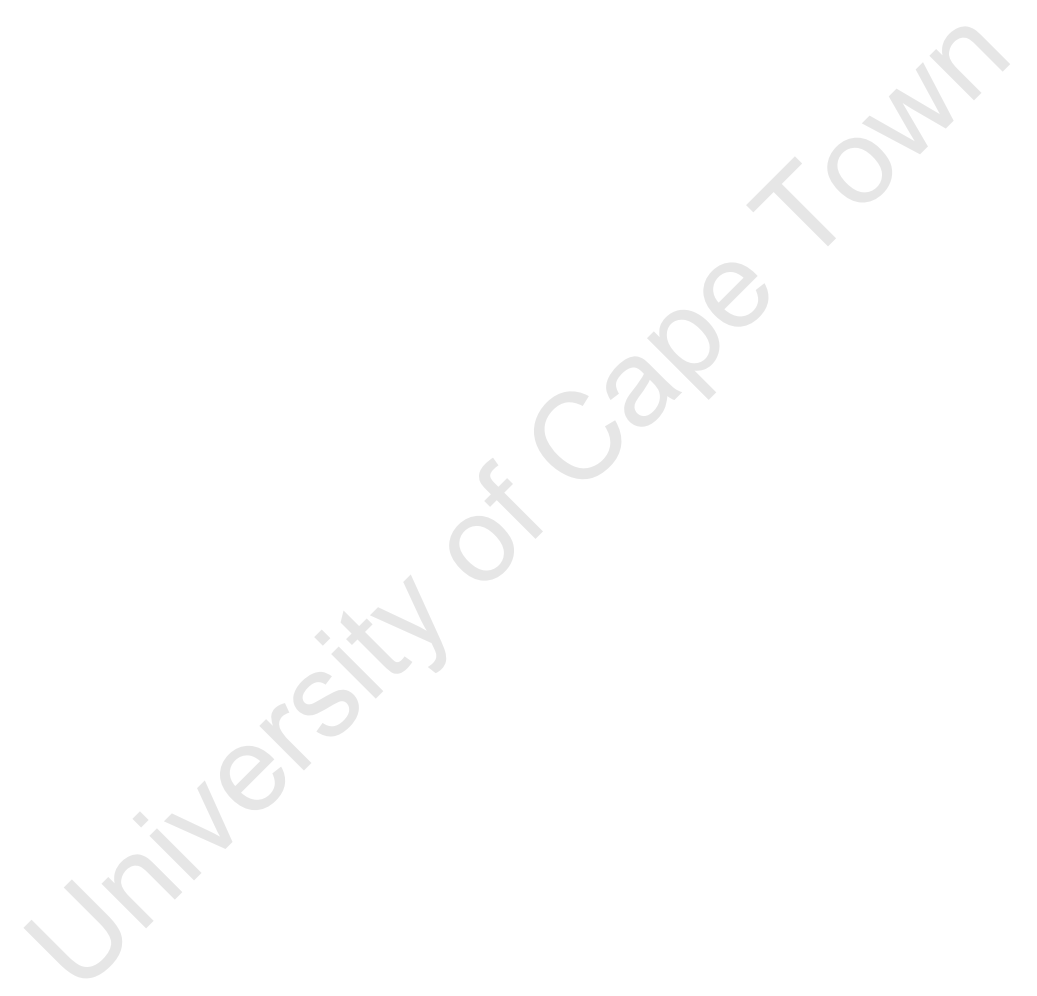




\section{Results}

This chapter starts by showing the results for the initial development stage. Next, the results for the full $3 D$ analyses are presented

\subsection{Model development}

Initial 2D manifold and cylinder models were used to develop the final 3D model. This section presents some of the important results generated during this phase.

Important aspects that were verified during this phase included:

- material property models - in particular iso-octane;

- droplet evaporation models;

- dynamic modelling of valve and piston motion;

- UDF for liquid fuel injection for stoichiometric AFR;

- mixture temperature control for the MON tests.

The initial 2D manifold model was used to test the material property models and spray evaporation modelling. The fuels and model parameters that were used were chosen to represent the work by Moran in [7, 8] and can be found in Section 4.1. For these studies, initial mean diameters of $50 \mu \mathrm{m}$ were used, as determined from literature. The droplet sizes that resulted during the experiments are unknown. Simulations were run for both $n$-hexane and iso-octane, since these fuels (and also $n$-heptane) were considered in [7, 8] and would provide validation for two fuels, even though $n$-hexane was not considered for the $3 \mathrm{D}$ studies. These results also served as sub-model validation for further, more complex modelling.

Results for simulations using $n$-hexane with different initial droplet diameters, and using the 
weakly coupled solver 1 , are shown in Figure 5.1. Average gas temperature along the manifold is shown, with 0 corresponding to the point of fuel injection. Material property models were available for $n$-hexane in the FLUENT ${ }^{T M}$ database.

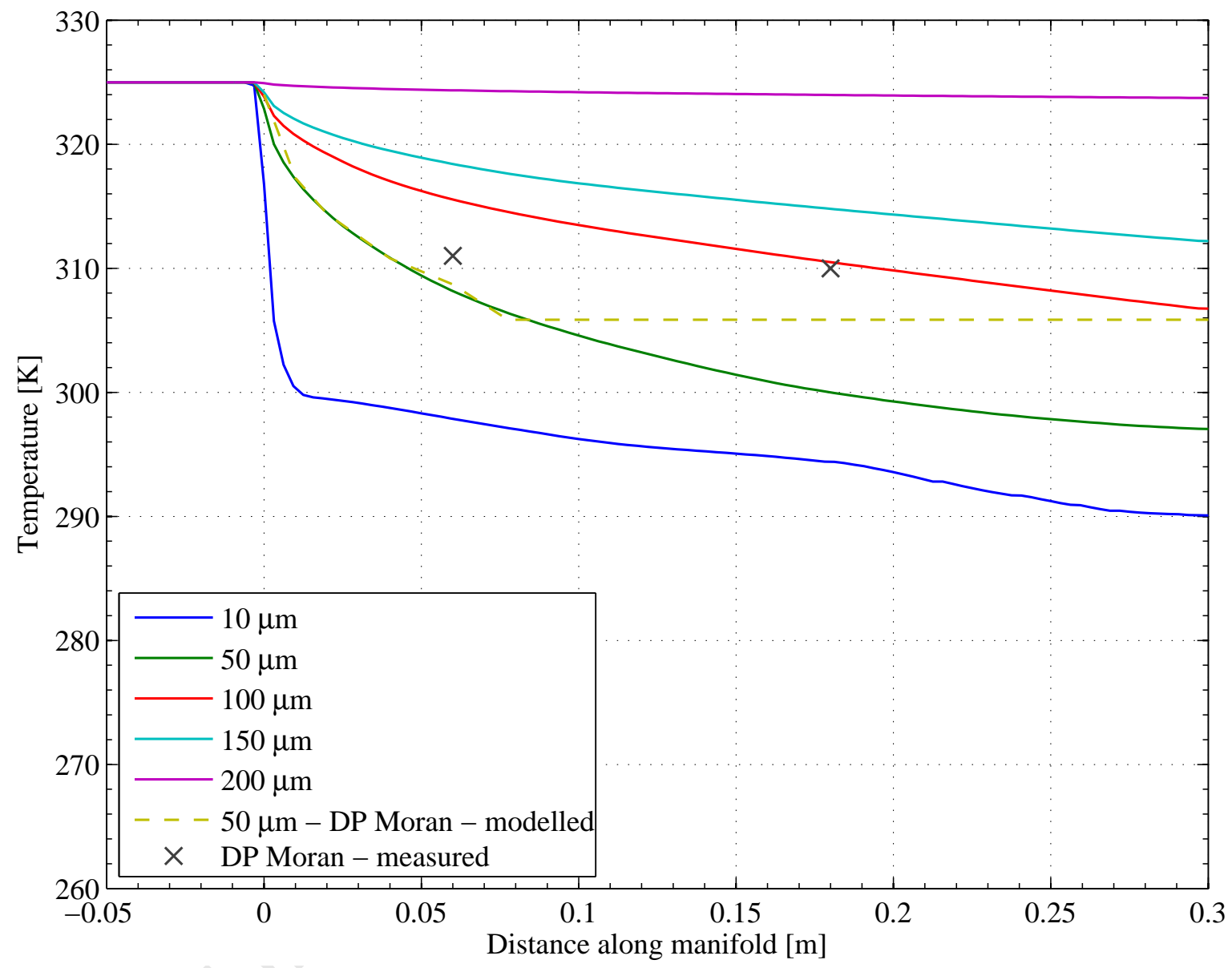

Figure 5.1: Average static temperature of the fuel-air mixture in the manifold for n-hexane liquid spray over a range of initial droplet diameters. The weakly coupled solver was used.

It can be seen that the cooling speed decreases with increasing droplet diameter, as would be expected. Also, at $10 \mu \mathrm{m}$, most of the liquid evaporates very rapidly. This is seen as the gas is cooled initially with a noticeable plateau. For the other droplet diameters, evaporation (and hence cooling) occurs steadily with distance along the manifold.

Figure 5.2 shows the data for the CFD simulation with strong and weak coupling together with the data from [7] for iso-octane fuel.

\footnotetext{
${ }^{1}$ Weakly coupled implies that the flow field is solved, followed by the particle tracks, then again the flow field. Strongly coupled implies that once the flow field is solved, the particle tracks and flow field are solved iteratively until both have converged to a final solution.
} 


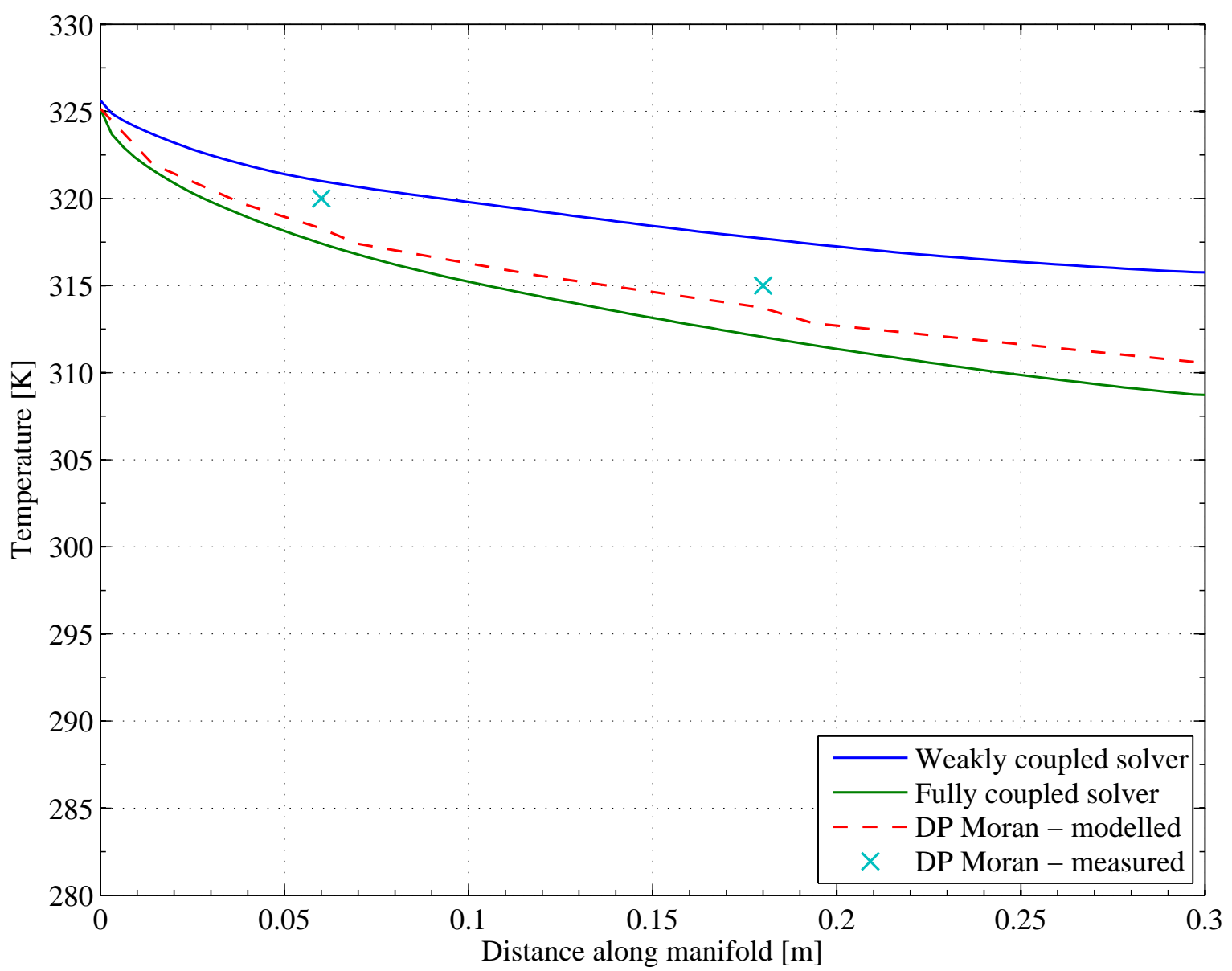

Figure 5.2: Average static temperature vs. distance along manifold for iso-octane

The data from [7] is seen to be bounded by the data from the weak and strongly coupled simulations. The rate of cooling follows the same trend as that of [7]. The weakly coupled case under predicts and the strongly coupled case over predicts cooling compared to [7]. The unsteady solver (which would be used for 2D planar and 3D simulations) is a hybrid between the two, and coupling between the phases occurs at regular time intervals.

For these tests a piecewise-linear approximation was used for fuel saturation vapour pressure. Mass diffusivity was assumed to remain constant. This option was chosen after simulations were performed with $n$-hexane with both constant and variable mass diffusivity functionality. The results for each case were identical, allowing the simpler and more computationally efficient option to be used. 


\subsubsection{AFR UDF}

The aim of the 2D planar model was to develop the dynamic mesh model, in-cylinder events and the fuel injection UDF. The 2D model did not incorporate a heater manifold, and hence did not ensure full evaporation of the liquid fuel, and should thus not be compared to the $3 \mathrm{D}$ case, presented further on in this thesis. The 2D model did however serve the purpose of developing the UDF, since developing it with the full 3D model, which included the heater element, was not practically an option due to the run-times associated with one single engine cycle.

The UDF (see Appendix An) which defines the mass flow rate of liquid fuel being injected at a given time was calibrated to deliver stoichiometric (or slightly rich) AFR of the unburnt fuel-air mixture. It is useful to express the actual AFR normalized with the AFR that would result for stoichiometric mixing. This parameter is known as the equivalence ratio $(\lambda)$ given by:

$$
\lambda=\frac{A F R_{\text {actual }}}{A F R_{\text {stoiciometric }}}
$$

When $\lambda>1$, it implies that the fuel-air mixture is lean of stoichiometric; when $\lambda<1$, the fuel-air mixture is rich of stoichiometric. For the results presented here, the in-cylinder $\lambda$ was calculated using only gaseous components and measured as a volume average over the cylinder volume. It would thus change as fuel evaporated if liquid fuel entered the cylinder. Figure 5.3 shows the time history of $\lambda$ under RON and MON conditions with iso-octane. Important stages of the intake dynamics are indicated on the graph. 


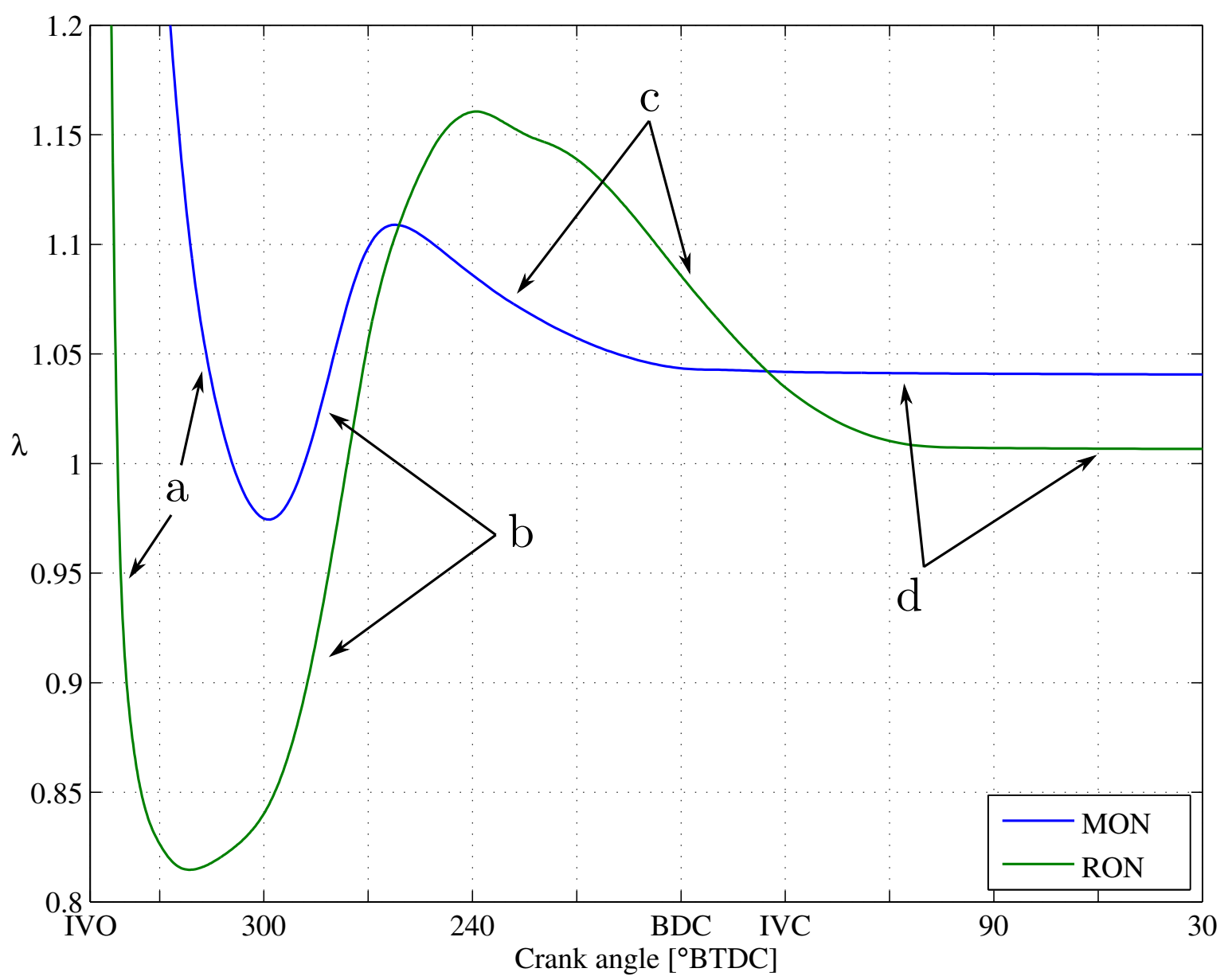

Figure 5.3: Average in-cylinder $\lambda$ vs. crank angle for RON and MON setup with iso-octane 
When the valve opens, there is no fuel-air mixture present in the cylinder. During stage (a), fuel vapour and evaporating fuel liquid enter the cylinder, changing the in-cylinder gas from lean to rich. At the same time air flows into the cylinder, effectively reducing this effect. At some point an equilibrium is reached whereafter $\lambda$ starts increasing, since a greater mass of air is flowing into the cylinder (stage (b)). During stage (c) air and fuel vapour is still flowing into the cylinder, and liquid fuel already in the cylinder is evaporating, decreasing the value of $\lambda$. Eventually all the liquid fuel in the cylinder evaporates, and no more fuel-air mixture flows into the cylinder and $\lambda$ remains constant (stage (d)).

The steady state $\lambda$ values for the two test configurations are different, with that for MON being leaner than for RON. The difference in values are less than $5 \%$. The important thing to note is that, in both cases, the steady state $\lambda$ values are lean of stoichiometric, and this could be a result of some of the fuel depositing on the wall and not reaching the cylinder, and also on dynamic flow effects.

The in-cylinder unburnt fuel-air mixture at $30^{\circ} \mathrm{BTDC}$ is slightly lean of stoichiometric. In reality the CFR engine runs at a slightly rich fuel-air mixture, since this yields the highest propensity for knocking. For the full 3D models, this was achieved by injecting a fuel liquid mass slightly higher than that required to achieve a stoichiometric ratio. This would also account for liquid fuel remaining in the inlet manifold and port.

\subsubsection{MON mixture temperature calibration using the 3D model}

The heating element used during the MON test was modelled as 4 cylindrical probe surfaces with a fixed wall temperature BC. The intention was not to fully model the heater elements. Rather, this method was chosen to ensure that the mixture temperature be controlled to $422 \mathrm{~K}$ at the port inlet. The correct geometry of these heater probes would result in the correct modelling of the flow dynamics in the manifold. In reality the mixture temperature is controlled by time averaged temperature readings at the mixture control location over many hundreds of engine cycles, which is unrealistic in terms of computational resources and cost. The temperature, and thus heat flux, used on the manifold was determined iteratively, and not by consideration of the electrical power and efficiency of the physical heating element used on the engine. The heater temperature boundary condition (HT BC) required to ensure a mixture temperature of roughly $422 \mathrm{~K}$ was found by an iterative method. Temperature was monitored at the thermocouple (probe) location, as well as the plane at which it was placed. The heating manifold is shown in Figure 5.4 with the areas of interest clearly indicated. 


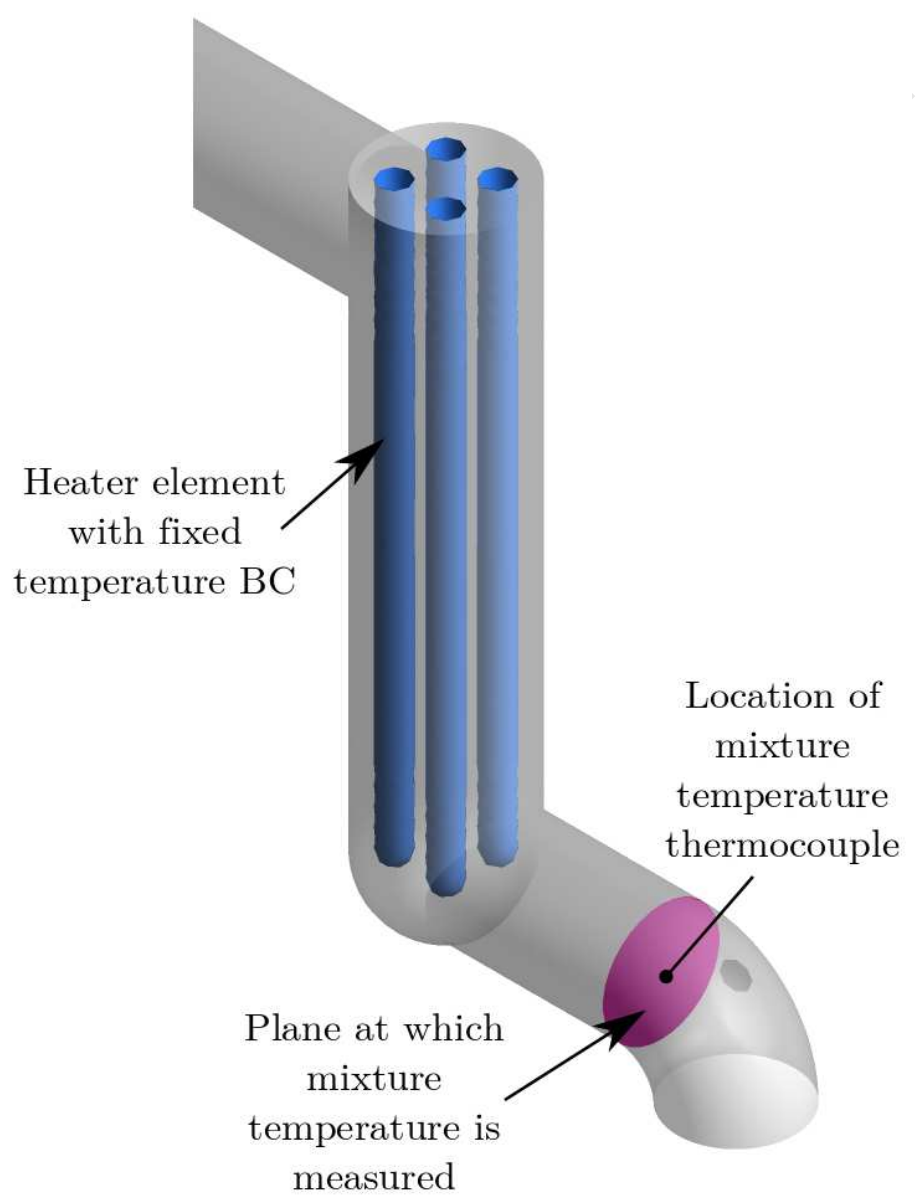

Figure 5.4: MON heating manifold and mixture temperature measurement location 
Three HT BCs were chosen for the motored MON test to see what inlet air temperatures resulted at the port inlet. The resulting time-averaged 2 air temperatures at the inlet probe location and the inlet probe plane are shown in Figure 5.5.

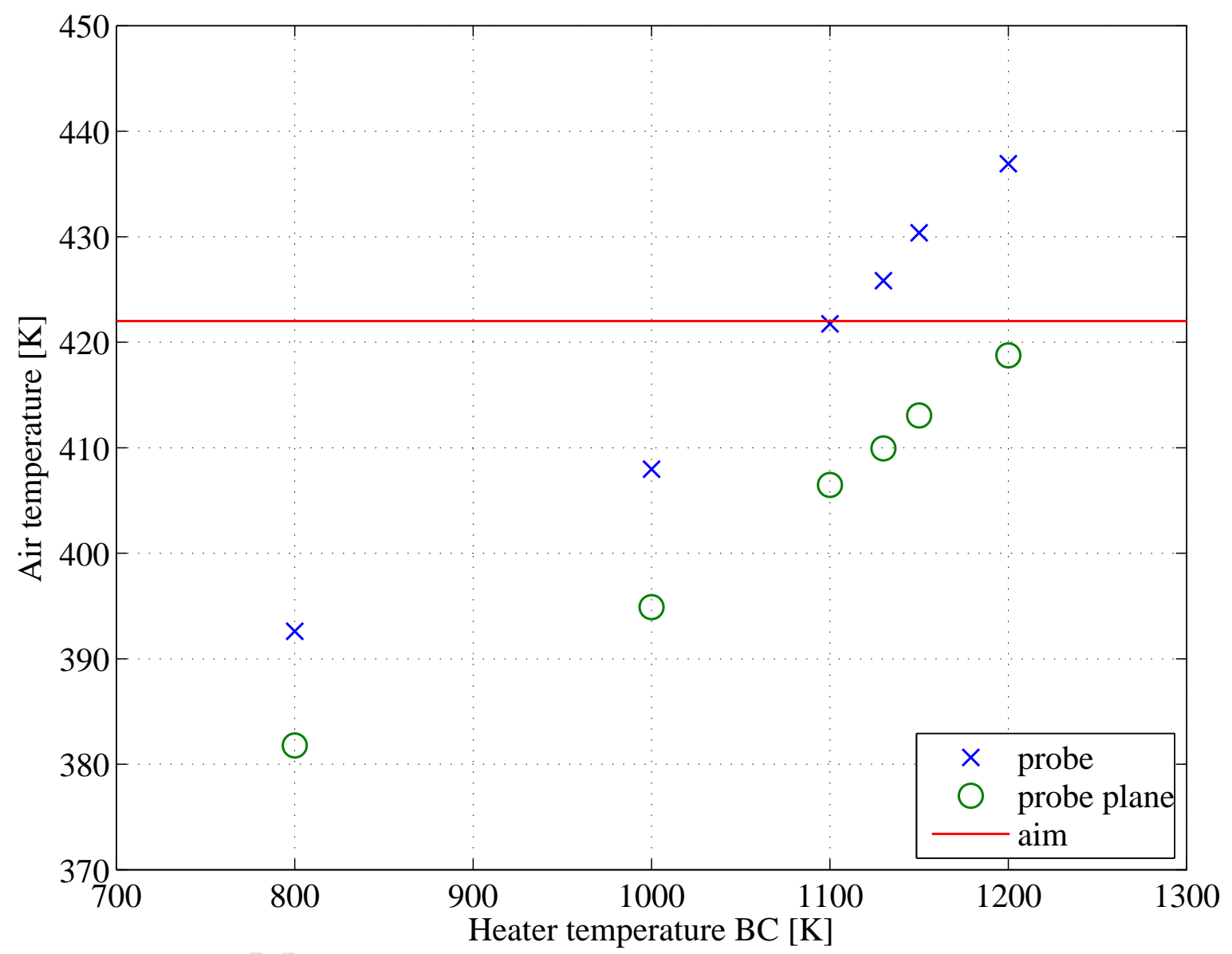

Figure 5.5: Heating element temperature $B C$ calibration for motored case with air

The probe temperatures are generally higher, by roughly $15 \mathrm{~K}$, than the average temperature over the probe plane. This is due to the lower manifold wall temperature which set up a thermal gradient across the plane.

Since the thermocouple used to control the mixture temperature is placed at the centre of the manifold, the temperature at this location was used to determine if the HT BC was sufficient. It was decided that a mixture temperature between 0 and $5 \%$ greater than $422 \mathrm{~K}$ would be an acceptable range of variation 3 . This implied mixture temperatures of between $422 \mathrm{~K}$ and $429 \mathrm{~K}$. For air, an HT BC of $1130 \mathrm{~K}$ resulted in an acceptable thermocouple position

\footnotetext{
${ }^{2}$ The temperatures shown were time-averaged over the intake stroke

${ }^{3}$ The $5 \%$ was calculated based on the centigrade scale, hence $5 \%$ of $149^{\circ} \mathrm{C}$.
} 
temperature.

Based on this, an initial estimate of $1250 \mathrm{~K}$ for the HT BC was chosen for the cases where liquid fuel was introduced. The higher HT BC was chosen to offset the evaporative cooling that would result when working with liquid fuel. This resulted in an average mixture temperature of roughly $436 \mathrm{~K}$ for iso-octane and $426 \mathrm{~K}$ for toluene. For toluene this was sufficient as it fell within $5 \%$ of the required $422 \mathrm{~K}$. In the case of iso-octane the mixture temperature was too high and hence the HT BC was changed until a suitable mixture temperature resulted. The results of these are shown in Figure 5.6.

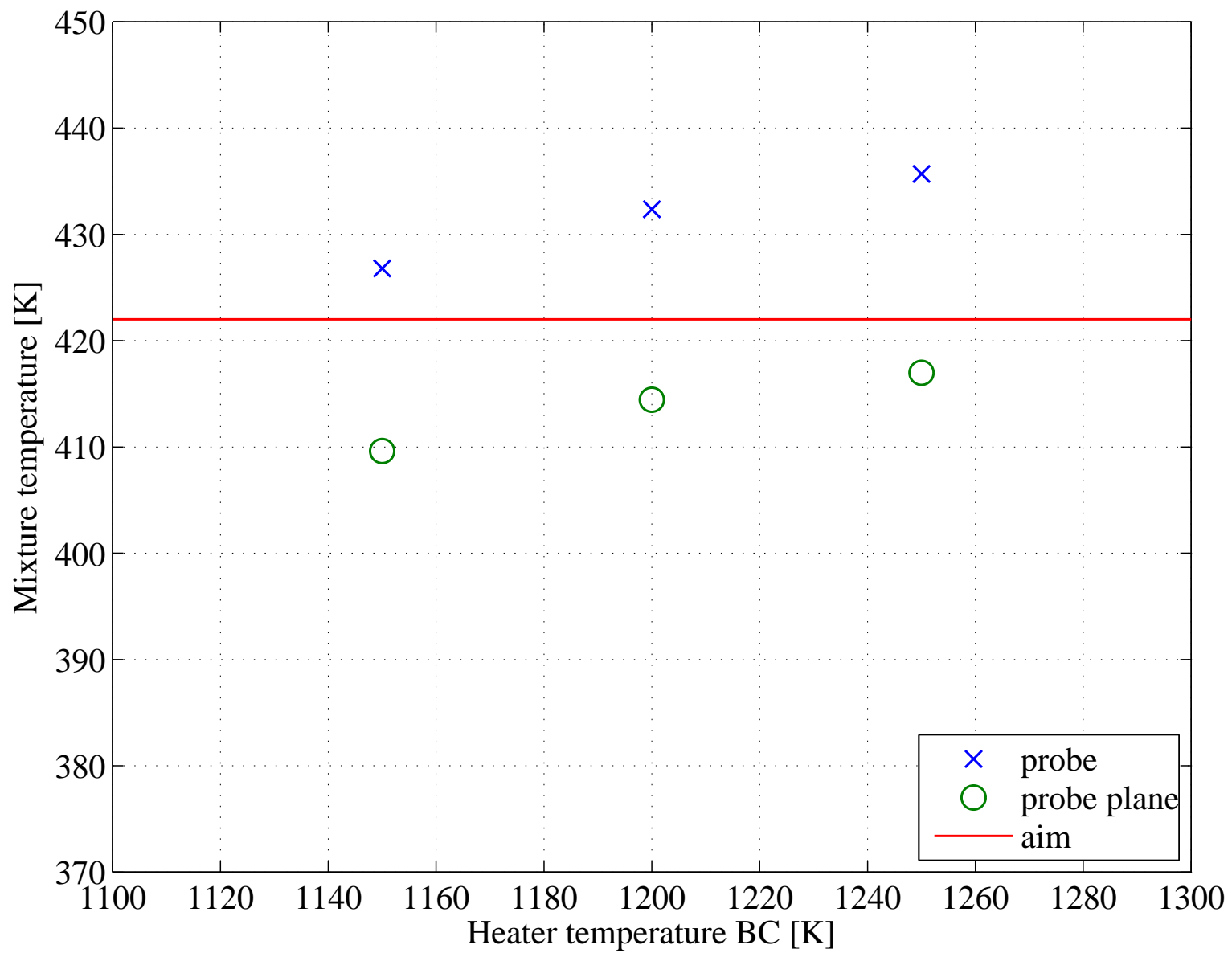

Figure 5.6: Heating element temperature $B C$ calibration for iso-octane-air mixture

Based on these results, a HT BC of $1150 \mathrm{~K}$ was chosen for iso-octane.

The resulting temperature gradients set up across the temperature measurement plane for iso-octane and toluene are shown at $90^{\circ}$ ATDC in Figure 5.7 to illustrate the reason for the 
difference in probe-location and probe-plane temperature values.

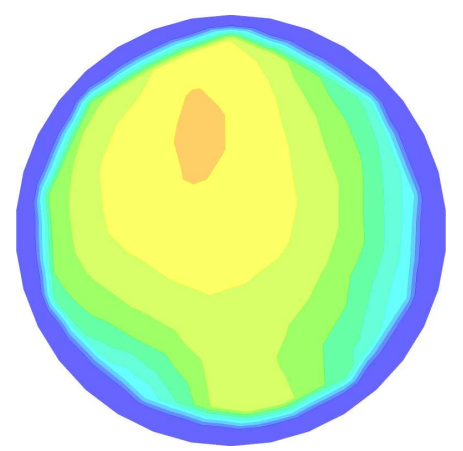

(a) air

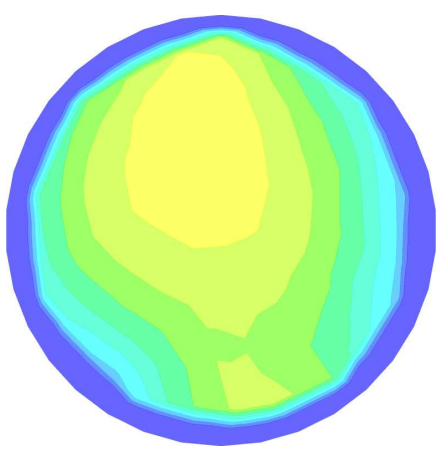

(b) iso-octane

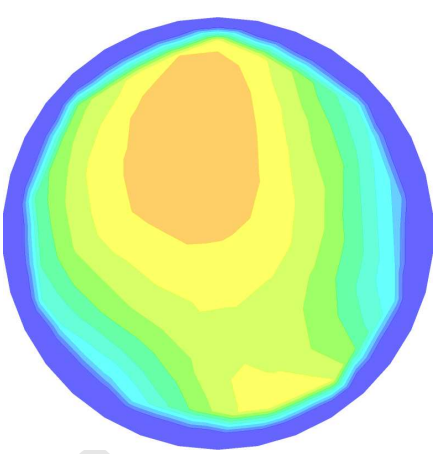

(c) toluene

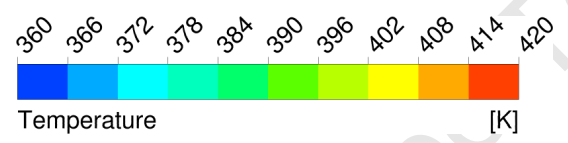

Figure 5.7: Temperature gradient across temperature measurement plane at 90 ATDC

It should be noted that these profiles represent a single snapshot in time (90 ATDC) and therefore the temperature at the probe location is not $422 \mathrm{~K}$, as is the case when the timeaveraged temperature at the probe location was considered. The final HT BCs that were chosen are presented in Table 5.1 .

Table 5.1: Final HT BC chosen for heating elements

\begin{tabular}{lccc}
\hline & HT BC & mixture T & deviation \\
\hline air & $1130 \mathrm{~K}$ & 426 & $2.7 \%$ \\
iso-octane & $1150 \mathrm{~K}$ & 427 & $3.4 \%$ \\
toluene & $1250 \mathrm{~K}$ & 426 & $2.7 \%$ \\
\hline
\end{tabular}

As shown above, the unburnt gas temperatures which results for the motored case and with both fuels is roughly $3 \%$ more than the required $422 \mathrm{~K}$, and was chosen to be satisfactory. These temperature BCs were used in all subsequent studies. 


\subsection{Results of the final 3D models}

Results are shown up to $30^{\circ}$ BTDC. This point was chosen before MON spark $\left(19^{\circ}\right.$ to $26^{\circ}$ BTDC) and well before RON spark (13 ${ }^{\circ}$ BTDC), to allow for direct comparison across both methods.

\subsubsection{Evaporative cooling}

For the MON tests, all the fuel was effectively evaporated before it entered the cylinder. In fact, on closer inspection it was noted that no liquid fuel reached the port, implying that all the fuel evaporated in the heater manifold. This section thus focuses on the RON tests, where significant concentrations of liquid fuel were present in both the port and cylinder.

Figure 5.8 shows the mass of in-cylinder fuel in vapour and liquid phase respectively for RON tests with iso-octane and toluene.

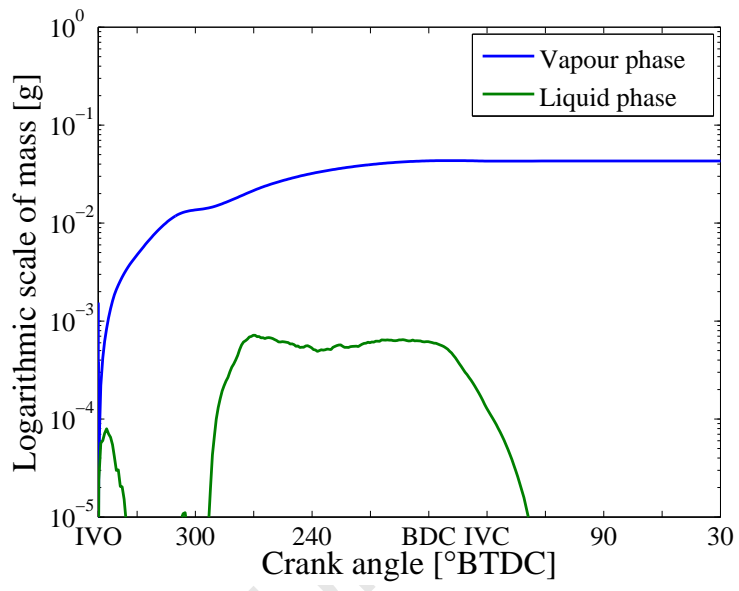

(a) iso-octane

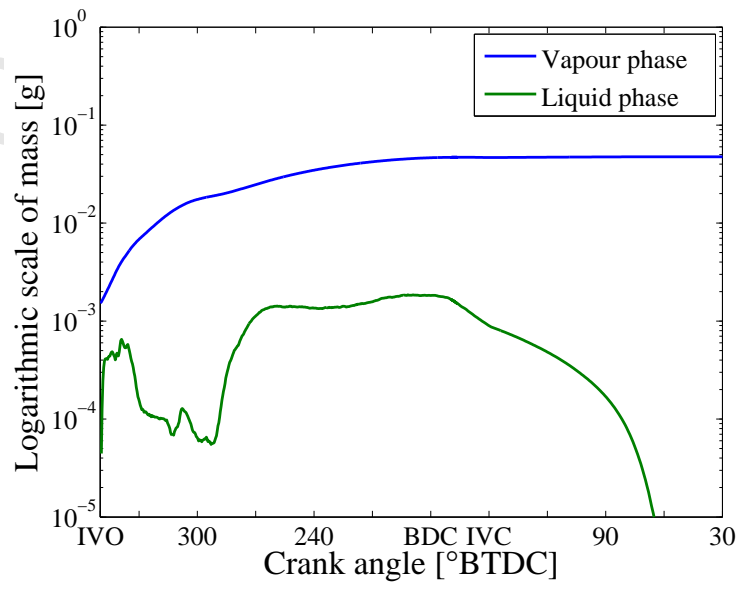

(b) toluene

Figure 5.8: Mass of in-cylinder fuel in vapour and liquid phase respectively vs. crank angle

In both cases, liquid fuel enters the cylinder. Also, there is still liquid fuel present in the cylinder at IVC. More liquid fuel is present for toluene than iso-octane. This was expected since toluene has a higher LHV and thus requires more energy to evaporate. The amount of fuel vapour in the cylinder increases as vapour flows into the cylinder, and as fuel liquid evaporates. At IVC no more mass enters the cylinder and the mass of fuel vapour increases very slightly as the remaining in-cylinder liquid fuel evaporates. Once all the fuel is evaporated, the mass of fuel vapour stays constant. This point is reached sooner with iso-octane than with toluene. Iso-octane is fully evaporated $25^{\circ}$ after IVC, while toluene is fully evaporated at $115^{\circ}$ after IVC. 
Based on these insights, there was thus a much greater potential for in-cylinder evaporative cooling to occur with toluene. The percentage of total fuel in liquid phase for toluene varied from $5 \%$ at $270^{\circ}$ BTDC to $1.8 \%$ at IVC. For iso-octane it varied from $3 \%$ at $270^{\circ}$ BTDC to only $0.4 \%$ at IVC. The question should be asked whether such small percentages could have a substantial effect on the in-cylinder temperature gradient and heat transfer. To put this into context, Figure 5.9 shows the mass of liquid fuel in the cylinder and intake port during intake and compression 4 .

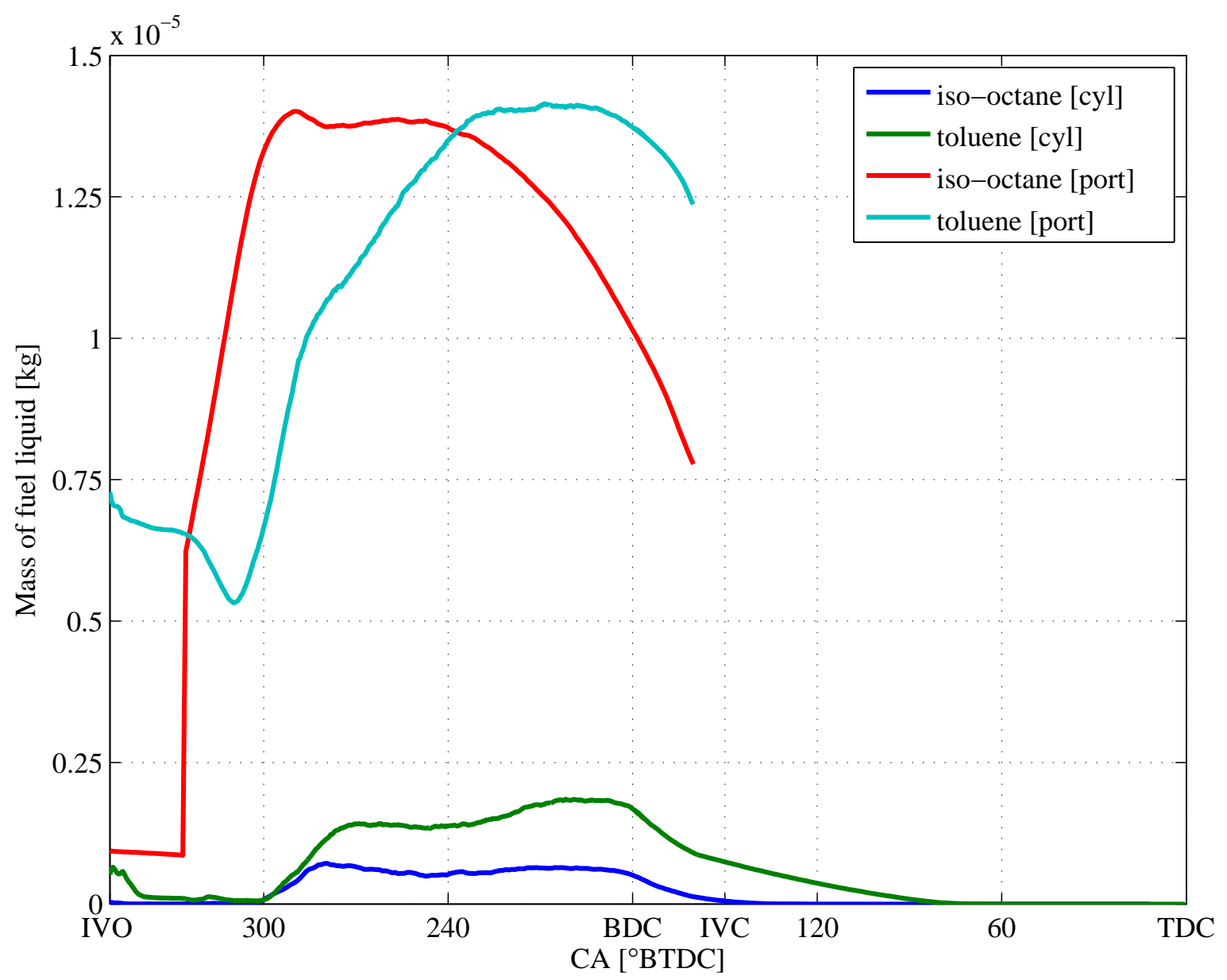

Figure 5.9: Liquid fuel in cylinder and port vs. crank angle for intake and compression

This indicates that most of the evaporation took place on entry to the cylinder. This makes sense as the high velocities through the valve, as well as the more hostile in-cylinder temperatures provide the necessary energy to evaporate the liquid fuel. For toluene, less of the fuel evaporated in the port compared to iso-octane, hence more liquid fuel was present in

\footnotetext{
${ }^{4}$ The port volume is defined as the volume from the manifold outlet interface to the cylinder interface as per Figure 4.11
} 
the cylinder at the time of IVC compared with iso-octane. The maximum cooling potential associated with the mass of liquid fuel in the port and cylinder was calculated by assuming that all the LHV of the liquid fuel, $m_{l}$, cools the gas, $m_{g}$, such that:

$$
\text { cooling potential }=\Delta T=\frac{m_{l} \mathrm{LHV}}{m_{g} c_{p}}
$$

The time-averages of these values over the intake stroke are given in Table 5.2

Table 5.2: Cooling potential of the liquid fuel in port and cylinder

\begin{tabular}{lcc}
\hline & Cylinder & Port \\
\hline Iso-octane & $0.17 \mathrm{~K}$ & $28.1 \mathrm{~K}$ \\
Toluene & $0.54 \mathrm{~K}$ & $86.8 \mathrm{~K}$ \\
\hline
\end{tabular}

The cooling potential of the in-cylinder liquid fuel is negligible. However, comparing the cooling potential of the toluene liquid in the port with that of iso-octane liquid in the port, it would be expected that the in-cylinder mixture for the toluene case be much lower than that for iso-octane. This is if one assumes that the energy required to evaporate this liquid is obtained from the surrounding gas, and not the surrounding wall surfaces.

The time-averaged surface heat flux is shown in Figure 5.10 for the intake manifold sections, the inlet port and the cylinder during both intake and compression. The values are normalized using that of motored air for each case. A positive surface heat flux implies that the average wall temperature is greater than the average temperature of the gas obtained within its boundaries. 


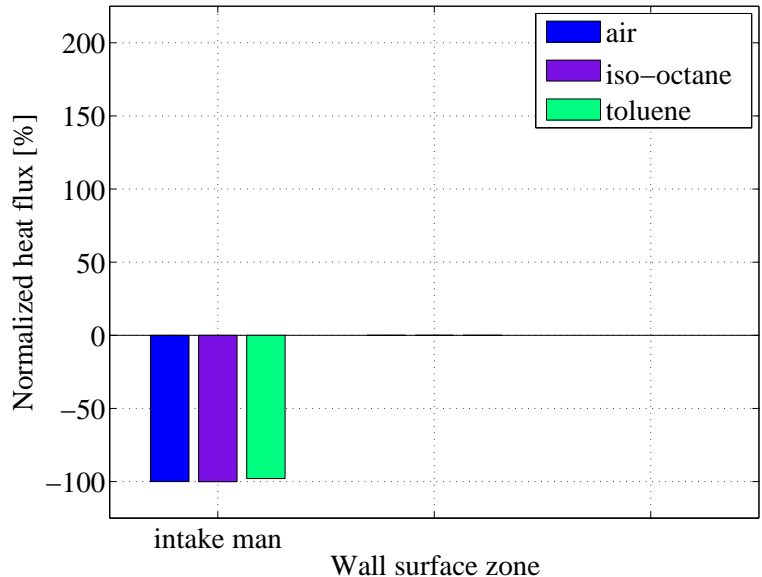

(a) RON - manifold

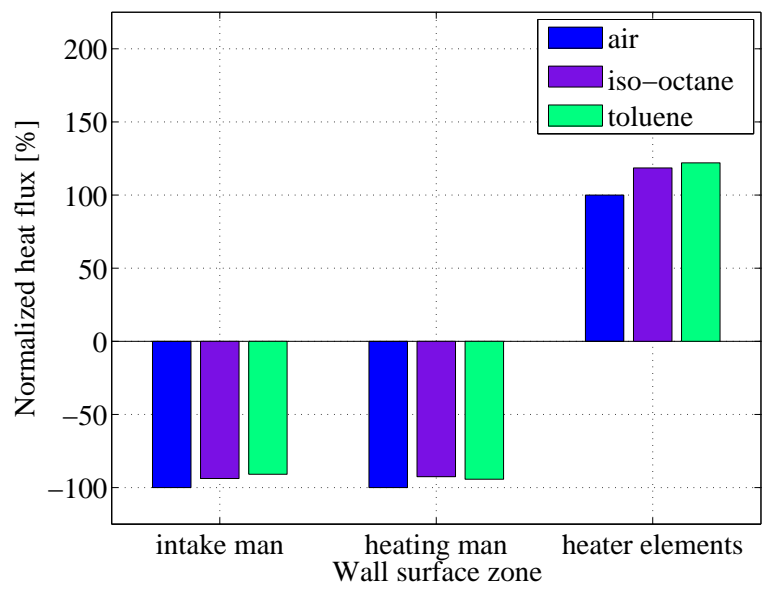

(c) MON - manifold

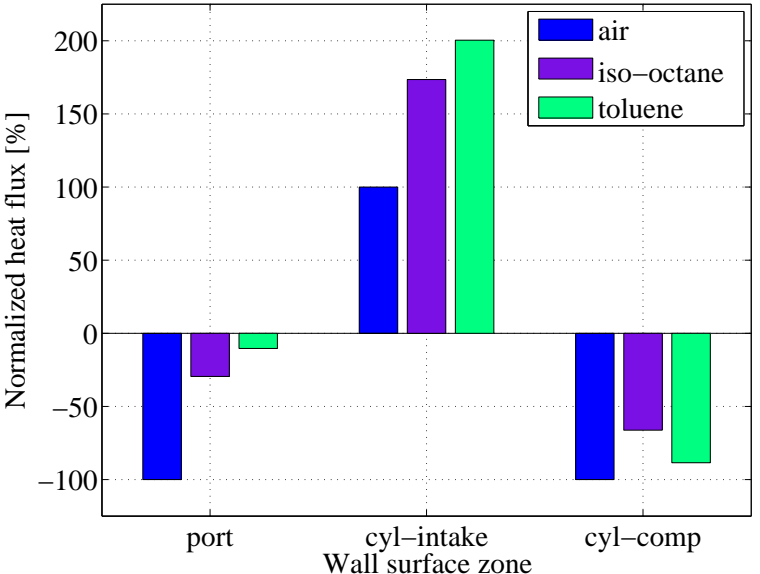

(b) RON - port and cylinder

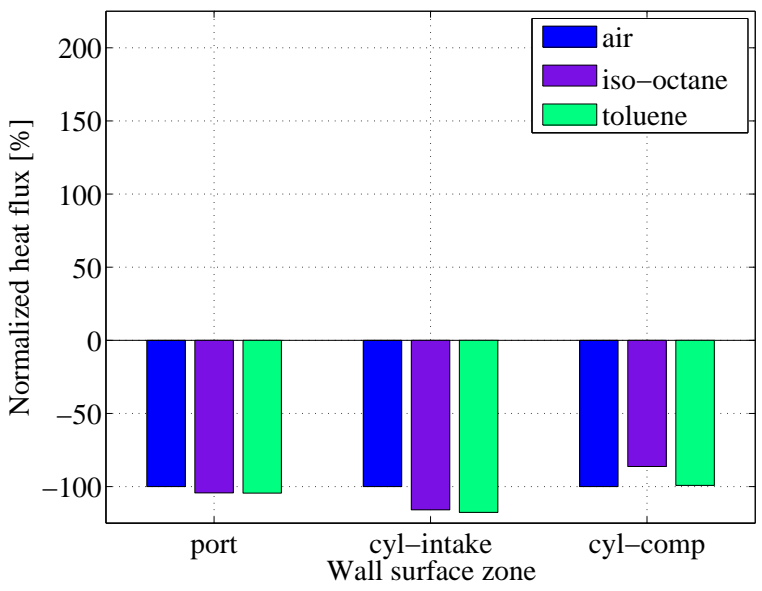

(d) MON - port and cylinder

Figure 5.10: Time average of total surface heat flux normalized with that for the motored case with air. A positive surface heat flux implies that on average, $T_{w}>T_{c} y l$, and vice versa. 
A clear difference is observed in the heat transfer characteristics for the port and cylinder (intake) when comparing RON and MON. For MON, the heat transfer for the cases with fuel is similar to that of the motored case in these zones. However, this is not the case for RON.

The port wall heat flux is negative for all cases. This implies that energy (heat) was 'removed' from the wall to maintain the temperature $\mathrm{BC}$ of $300 \mathrm{~K}$. If the motored case corresponds to $100 \%$ heat energy, for iso-octane $30 \%$ of that is required, and for toluene less than $10 \%$. This difference could be attributed to the latent heat transfer associated with the liquid fuel. Since toluene has a much higher LHV, the fuel-air mixture would be at a lower temperature, reducing the temperature gradient between the port wall and gas.

During intake the cylinder walls are hotter $(400 \mathrm{~K})$ than the in-cylinder gas. This implies that energy had to be 'added' to the wall to maintain the temperature BC of $400 \mathrm{~K}$. For the case with iso-octane, about $75 \%$ more energy is required compared to the motored case; for toluene double the amount of energy is required. This was expected since the toluene-air stream coming in is at a lower temperature because of the evaporative cooling that occurs in the port and as the liquid fuel evaporates within the cylinder.

At the start of compression, the toluene-air mixture is cooler than the iso-octane-air mixture, resulting in greater overall wall heat transfer. During compression however, the rank changes, and for iso-octane less energy is required to maintain the HT BC compared to toluene. The iso-octane-air mixture starts at a higher temperature at BDC than the toluene-air mixture. It would thus be expected that the iso-octane-air mixture heat transfer be more similar to the motored case heat transfer. However, the opposite is observed, with the toluene-air-mixture heat transfer being more similar to the motored case heat transfer. This indicates that the compression heating behaviour of the fuel-air mixture constituents significantly effects the temperature during compression. The adiabatic compression relationship is given by:

$$
P V^{\gamma}=\text { constant }
$$

The following relationship can be derived from the above in combination with the ideal gas laws:

$$
T_{2}=T_{1}\left(\frac{V_{1}}{V_{2}}\right)^{\gamma-1}
$$

Greater $\gamma$ values will thus result in greater temperature increases during compression for the same time increment. Figure 5.11 shows the values of $\gamma$ during compression for both fuels under RON and MON conditions. 

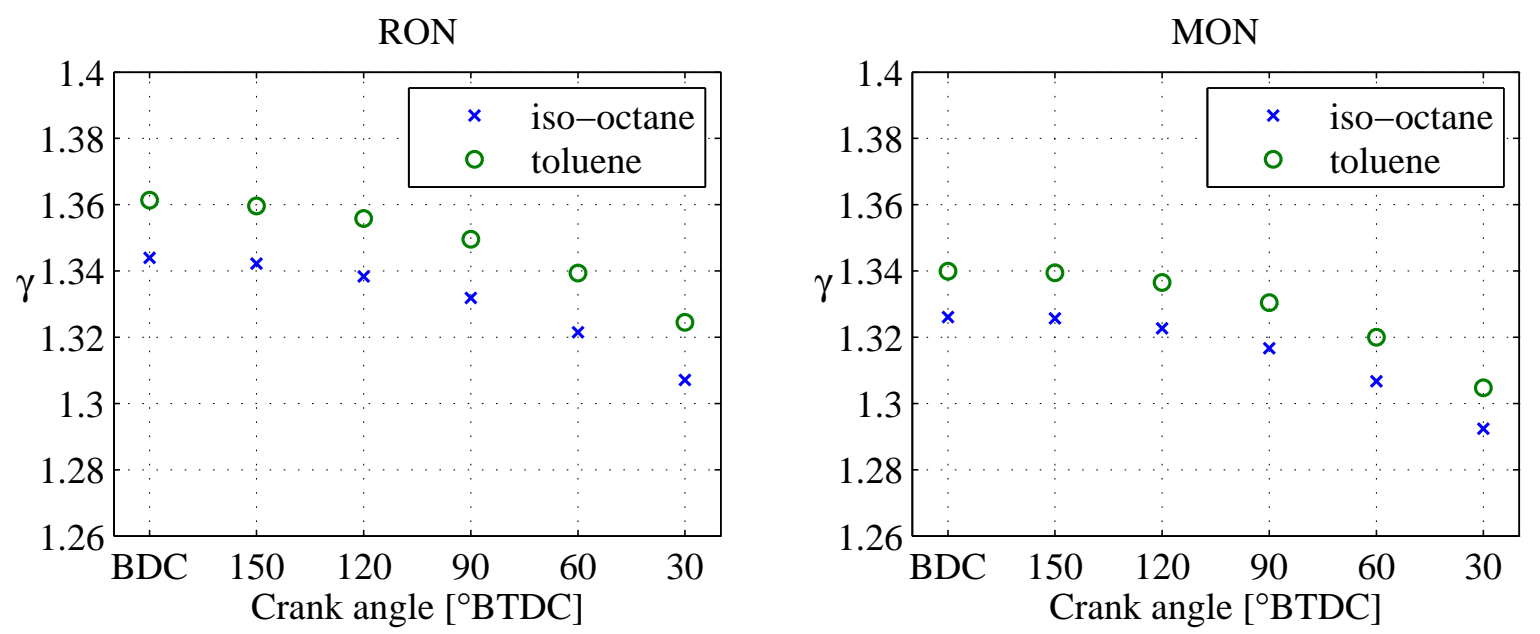

Figure 5.11: Fuel equivalence ratio, $\gamma$, vs crank angle during compression

Toluene exhibits higher $\gamma$ values over the compression cycle. This could explain the rank change of heat transfer from intake to compression. For air, $\gamma$ values range from 1.4 at $293 \mathrm{~K}$ to 1.37 at $600 \mathrm{~K}$. At $293 \mathrm{~K}$, Propane has a $\gamma$ value of 1.127 , and Pentane 1.07.

\subsubsection{In-cylinder temperature distribution}

The main aim of this study was to determine the in-cylinder temperature distribution set up during the octane rating of gasoline fuels, and to determine how the evaporative cooling which occurs under RON conditions affect these profiles. The temperature profiles are shown for both RON and MON test conditions in Figures [5.13] to [5.21. Temperature profiles are shown on 4 planes for each case. The first plane is the vertical cylinder mid plane. The other 3 are horizontal cuts at 3 different positions along the cylinder mid plane, as defined for the general case in Figure 5.12 . 


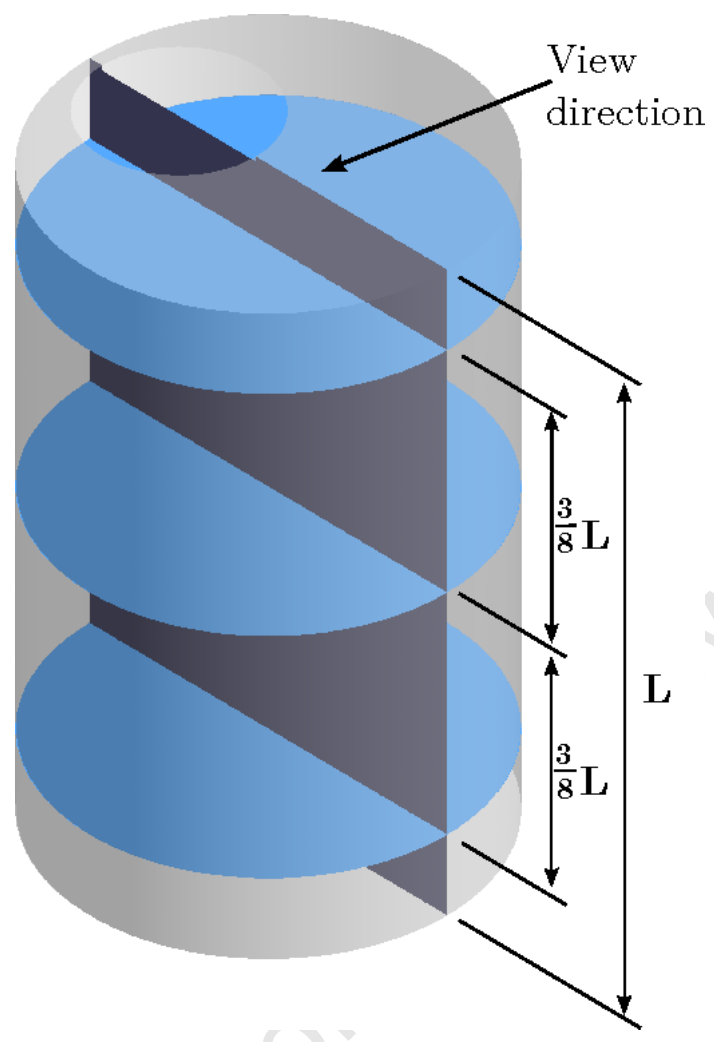

Figure 5.12: Locations of planes used for presentation of results

The in-cylinder temperature distribution at IVC is useful as initial conditions for thermodynamic engine models. These are shown for RON in Figure 5.13 and for MON in Figure 5.14. 


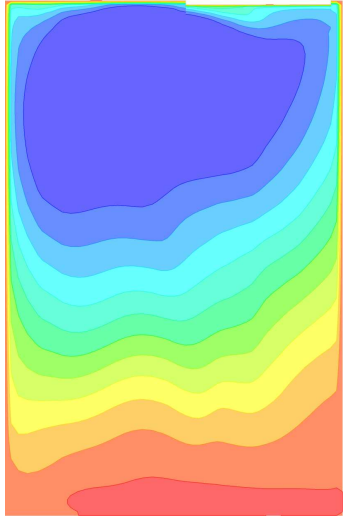

(a) air

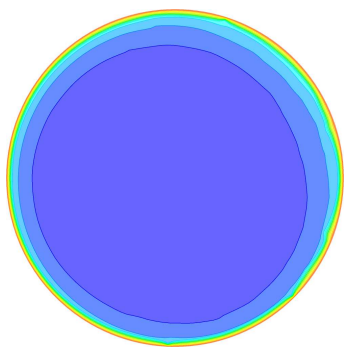

(d) air

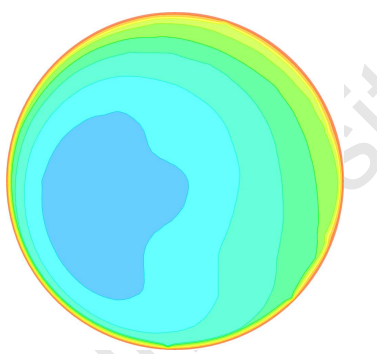

(g) air

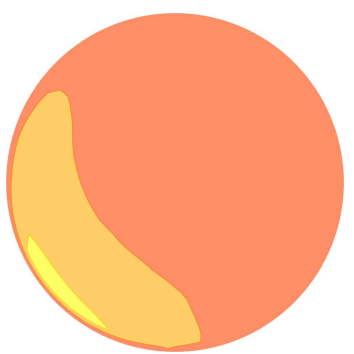

(j) air

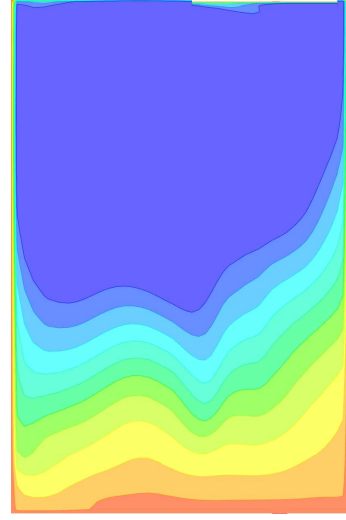

(b) $i$-oct

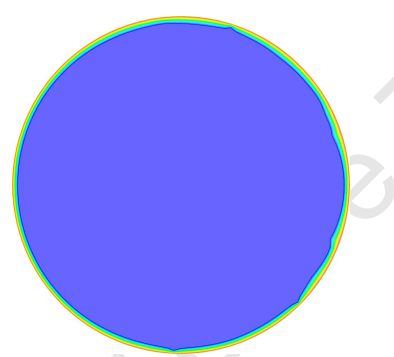

(e) $i$-oct

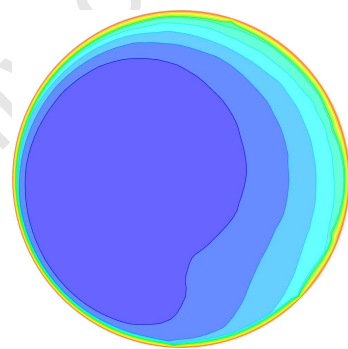

(h) $i$-oct

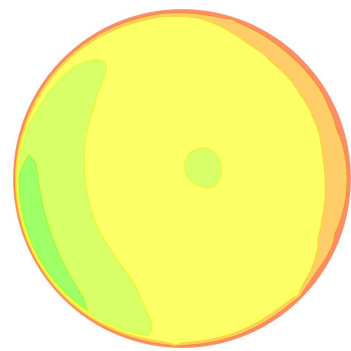

(k) $i$-oct

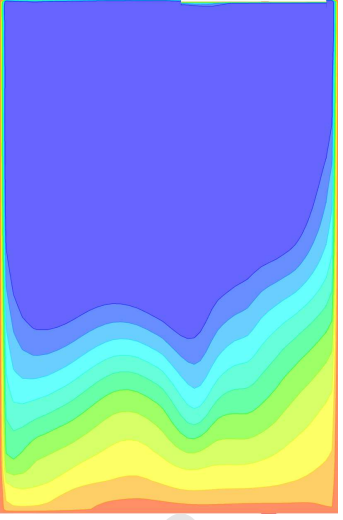

(c) tol

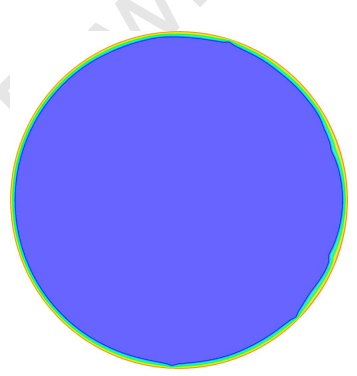

(f) $\mathrm{tol}$

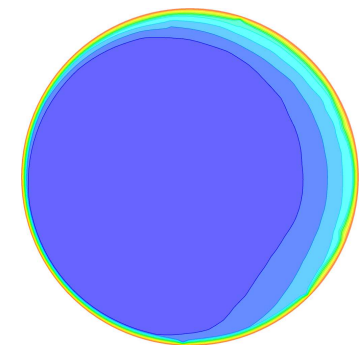

(i) $\mathrm{tol}$

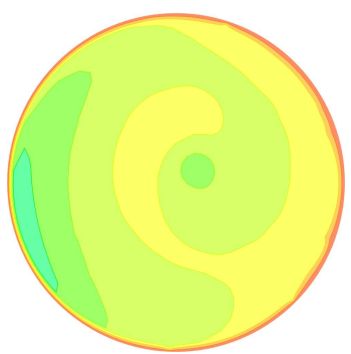

(1) tol

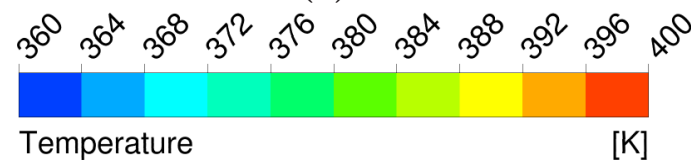

Figure 5.13: Temperature profiles for RON at IVC for the motored case with air, iso-octane and toluene. From top to bottom: vertical mid-plane, horizontal plane near vale, horizontal mid-plane and horizontal plane nearest to piston. 


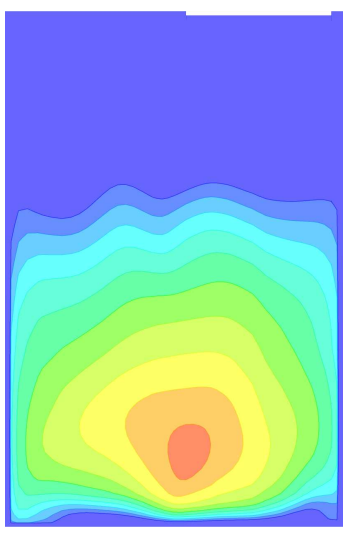

(a) air

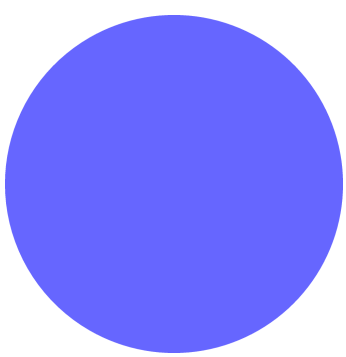

(d) $a i r$

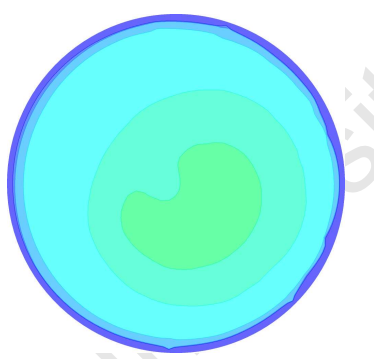

(g) air

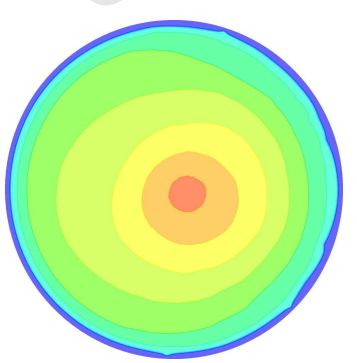

(j) air

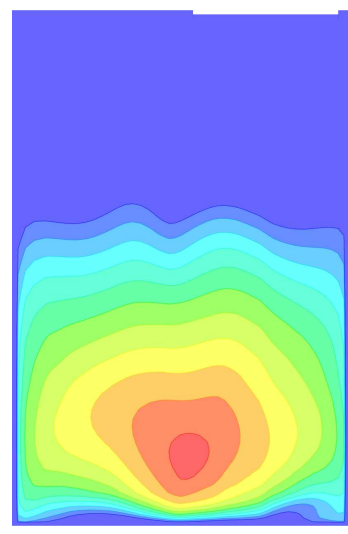

(b) $i$-oct

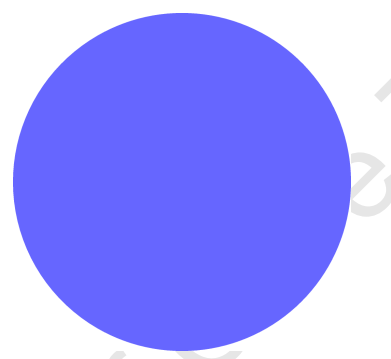

(e) $i$-oct

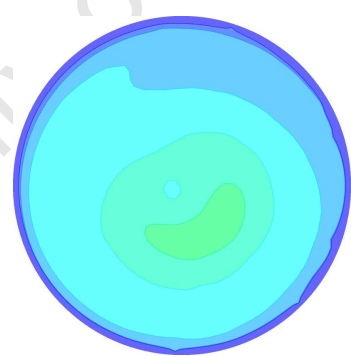

(h) $i$-oct

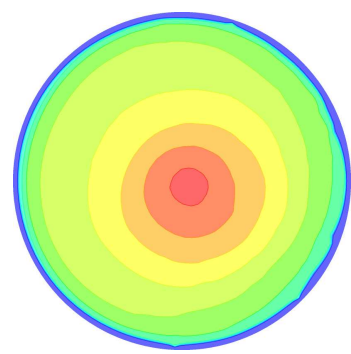

(k) $i$-oct

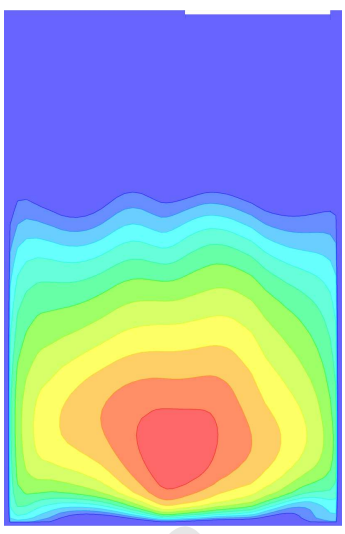

(c) tol

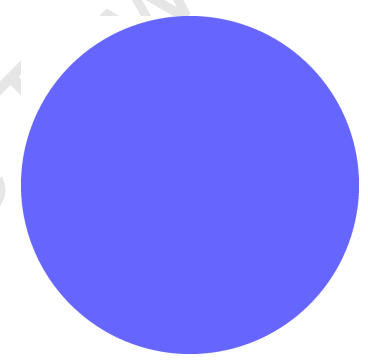

(f) $\mathrm{tol}$

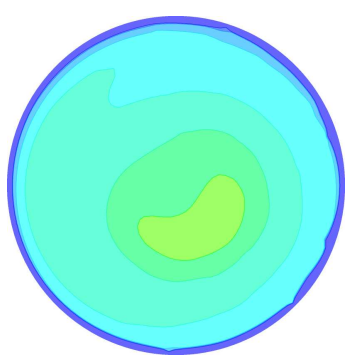

(i) tol

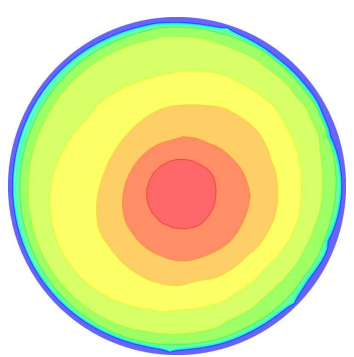

(l) tol

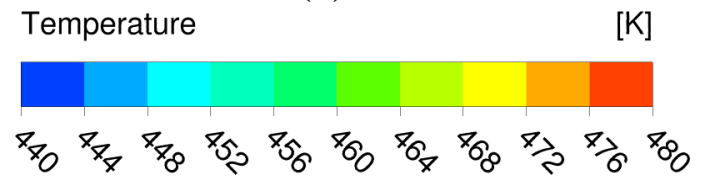

Figure 5.14: Temperature profiles for MON at IVC for the motored case with air, iso-octane and toluene. From top to bottom: vertical mid-plane, horizontal plane near vale, horizontal mid-plane and horizontal plane nearest to piston. 
Under RON and MON conditions, and for both fuels and the motored case, a strong vertical temperature gradient is observed at IVC. Such a strong vertical gradient was not obvious and not assumed in literature. In [21] it was assumed that the temperature gradient is set up radially.

The radial temperature profiles set up are symmetrical about the cylinder axis under MON conditions. Under RON conditions, the radial temperature profile are not symmetrical about the cylinder axis, as can be seen when considering the horizontal plane temperature contour plots. In [21] three different radial profile shapes were studied, however all of these were assumed to be axisymmetric. This would be sufficient for MON studies, however not for RON studies.

The asymmetrical profiles set up during RON can be attributed to the fuel evaporative cooling that took place as the liquid fuel entered the cylinder, as is shown in Figure 5.15 .
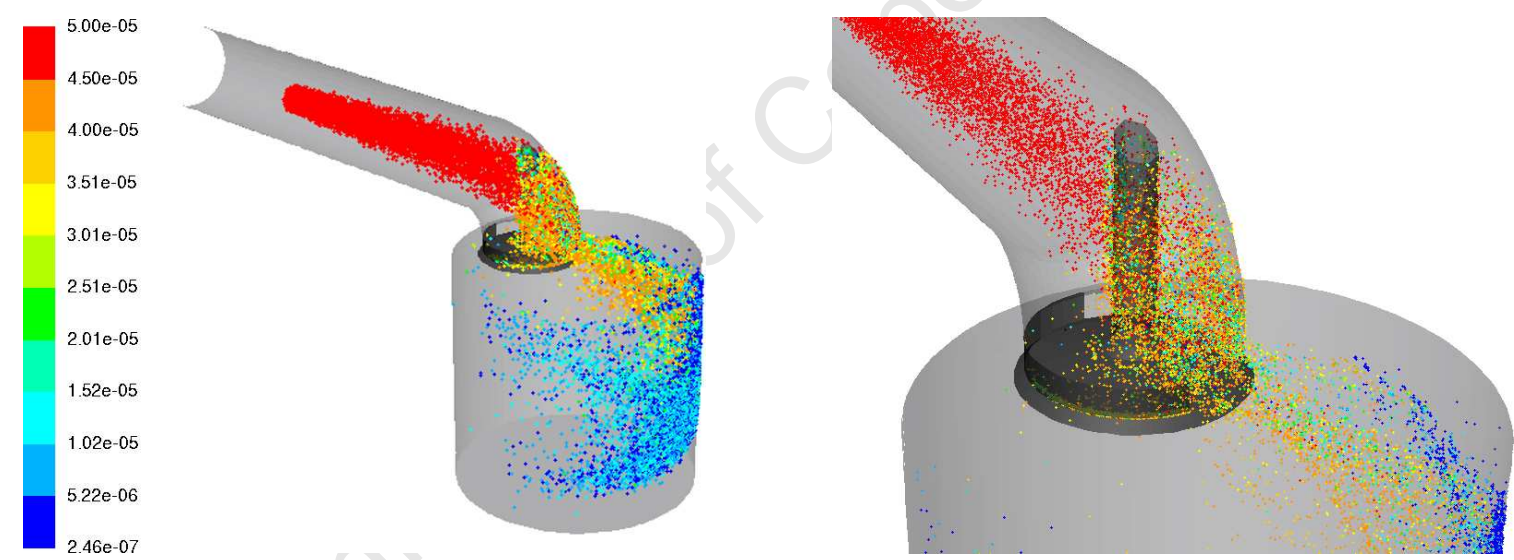

Figure 5.15: Iso-octane droplet tracks under RON conditions at $90^{\circ}$ ATDC, coloured according to droplet diameter $(\mathrm{mm})$.

The swirling flow in the cylinder directs the flow circumferentially along the cylinder sides, while the moving piston pulls it vertically down. The in-cylinder velocity distribution at the time of IVC is shown in Figure 5.16 for RON and in Figure 5.17 for MON. Similar velocity trends were found in [4], however it the test conditions at which these were shown was unclear. 

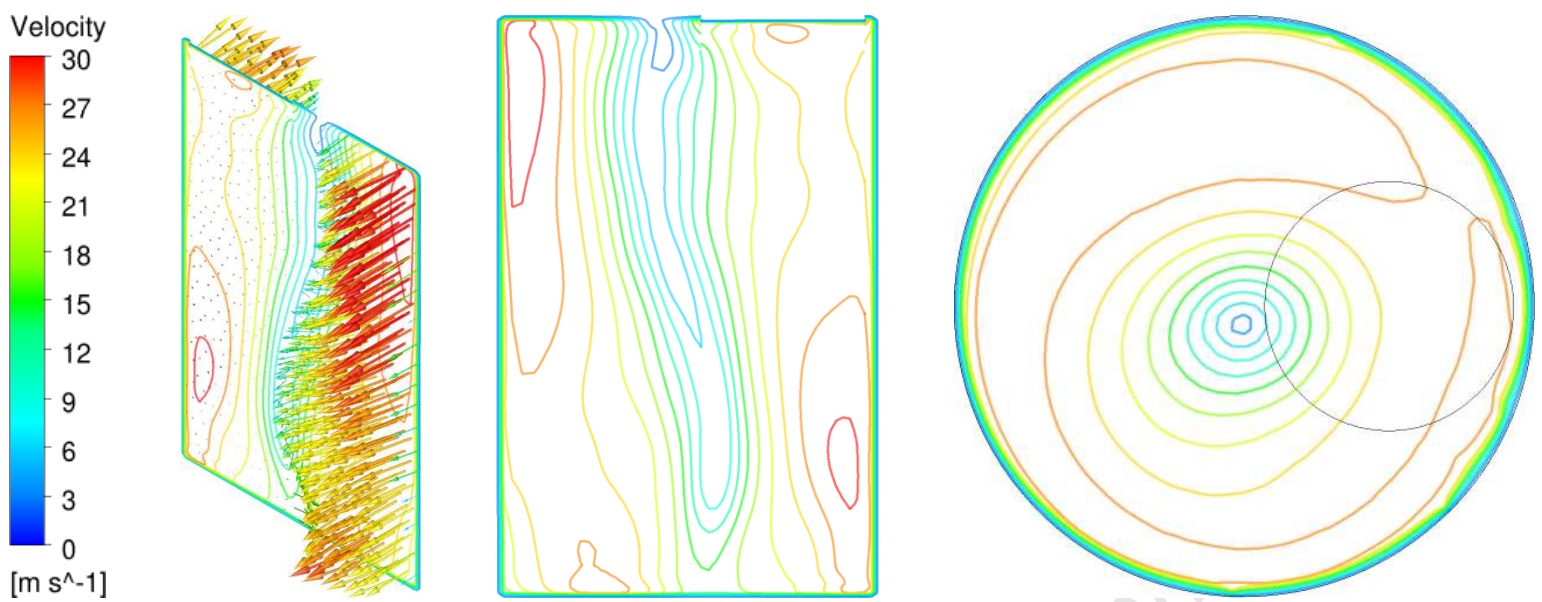

Figure 5.16: In-cylinder velocity distribution at IVC under RON conditions. Motored case. Vertical mid plane and horizontal mid-plane.
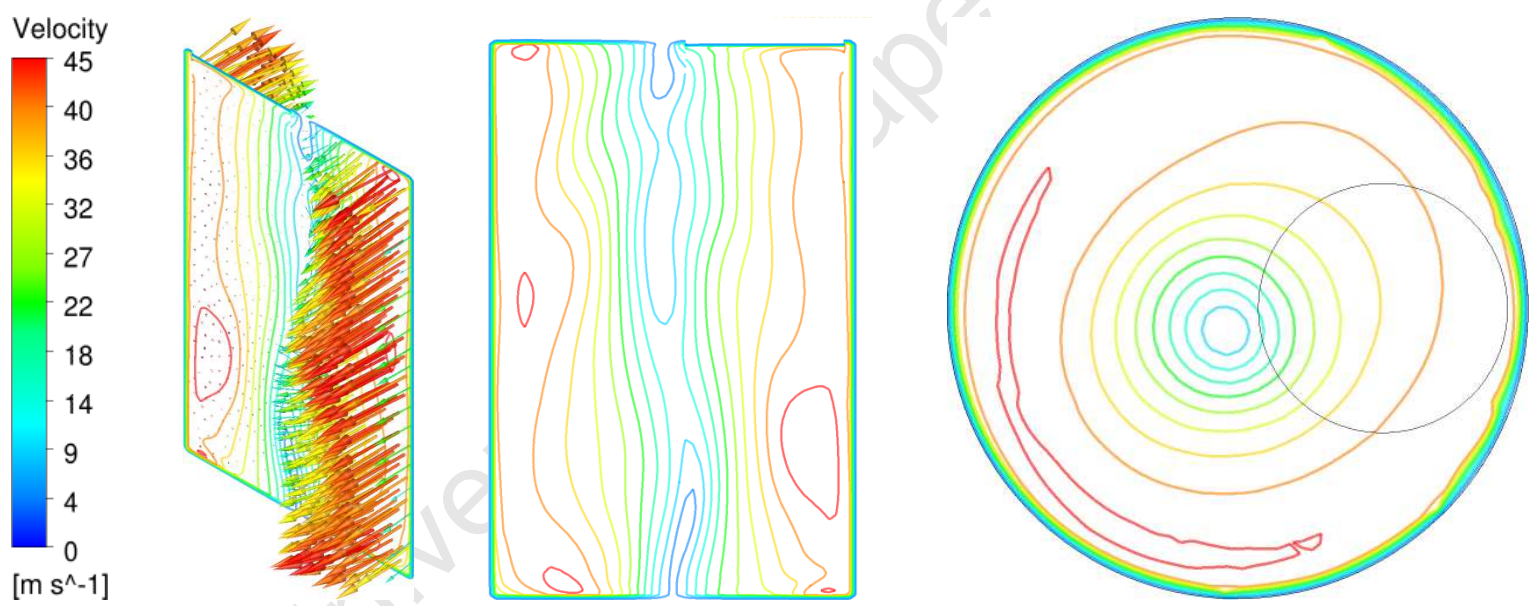

Figure 5.17: In-cylinder velocity distribution at IVC under MON conditions. Motored case. Vertical mid plane and horizontal mid-plane.

Under RON conditions the velocity profiles exhibit greater asymmetry than the MON. This would explain why the asymmetrical temperature distribution is also evident for the motored case with air, and implies that evaporative cooling is not the only factor contributing to the temperature asymmetry. The lower speeds associated with the RON test could also contribute as it would result in less in-cylinder turbulence compared to MON. Under MON conditions the higher engine speed - which results in increased in-cylinder turbulence and more developed in-cylinder swirling flow - diminishes such an asymmetric temperature profile. This was confirmed by considering in-cylinder velocity distribution and is shown at IVC in Figure 5.16 for RON and in Figure 5.17 for MON.

The in-cylinder temperature distributions at mid compression stroke (90 BTDC) are shown 
Chapter 5

in Figure 5.18 for RON and in Figure 5.19 for MON. 


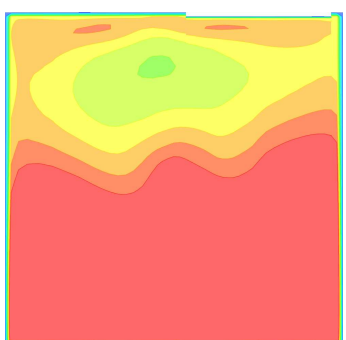

(a)

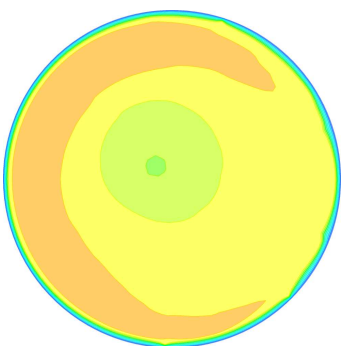

(d)

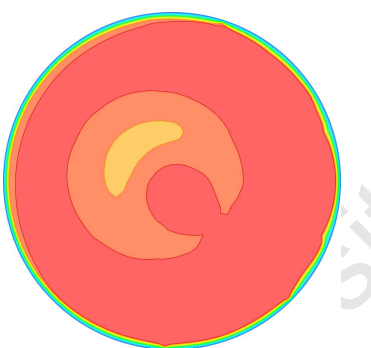

(g)

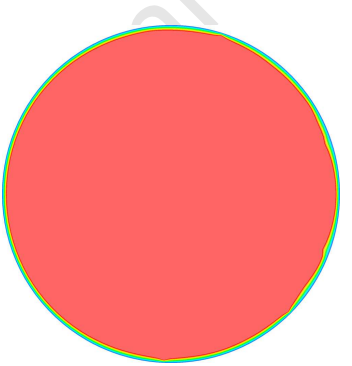

(j) air

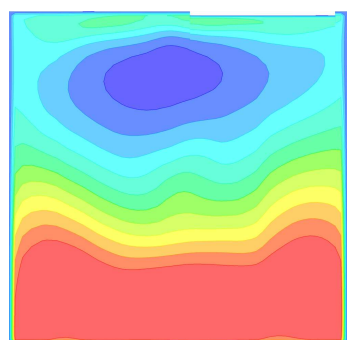

(b)

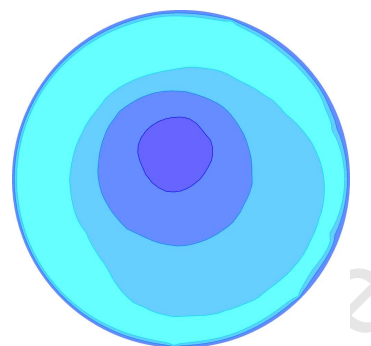

(e)

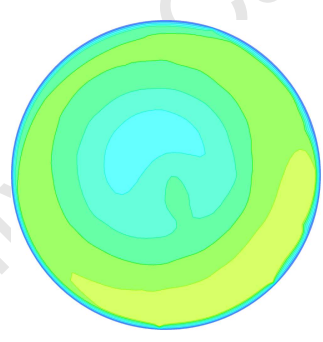

(h)

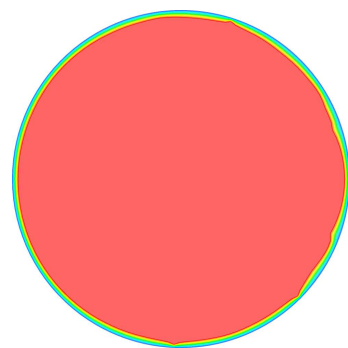

(k) $i$-oct

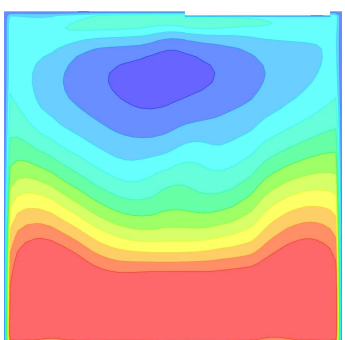

(c)

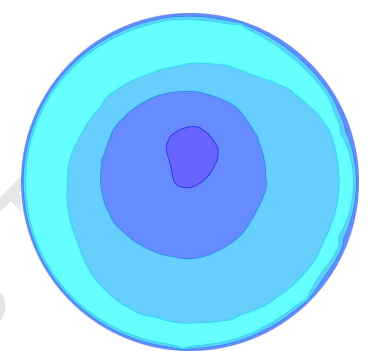

(f)

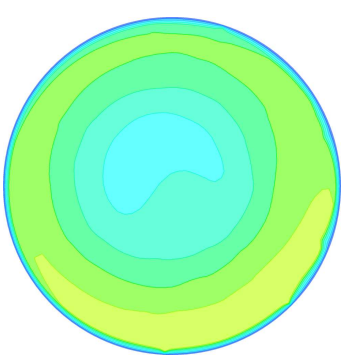

(i)

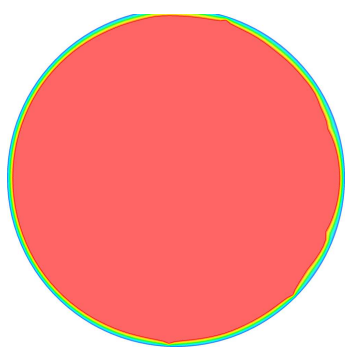

(1) tol

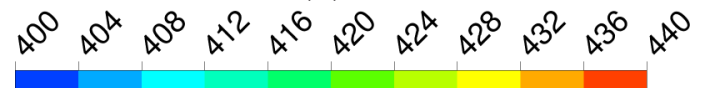

Temperature

$[\mathrm{K}]$

Figure 5.18: Temperature profiles for $R O N$ at $90^{\circ} A B D C$ for the motored case with air, iso-octane and toluene. From top to bottom: vertical mid-plane, horizontal plane near vale, horizontal mid-plane and horizontal plane nearest to piston. 


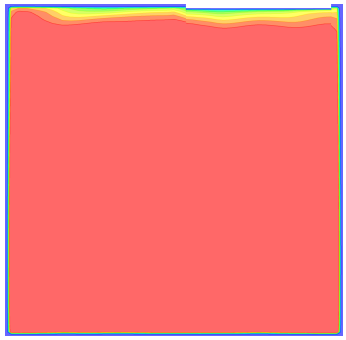

(a) air

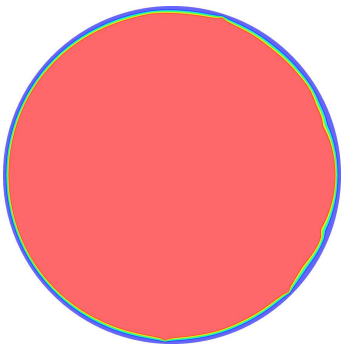

(d) air

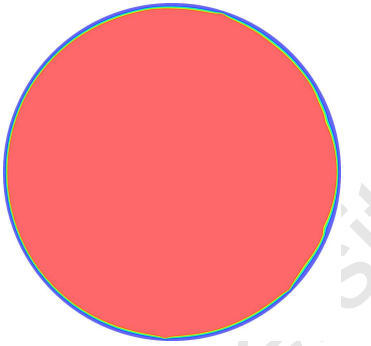

(g) air

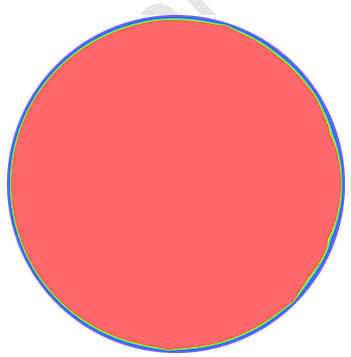

(j) air

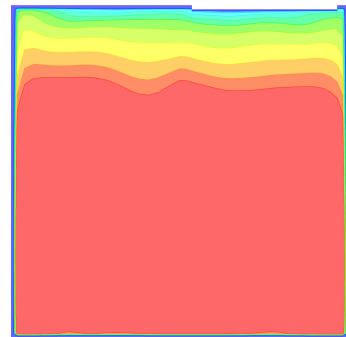

(b) $i$-oct

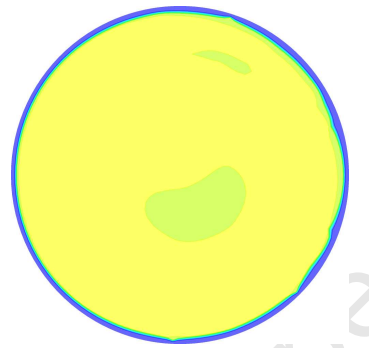

(e) $i$-oct

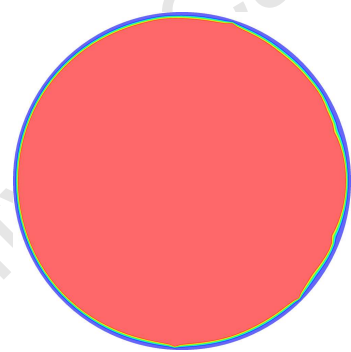

(h) $i$-oct

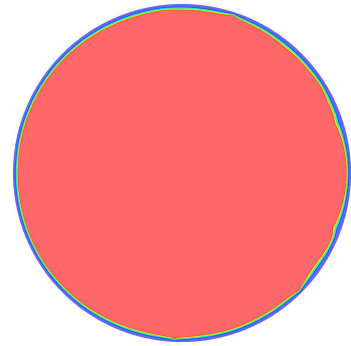

(k) $i$-oct

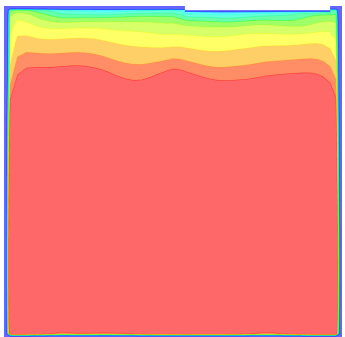

(c) tol

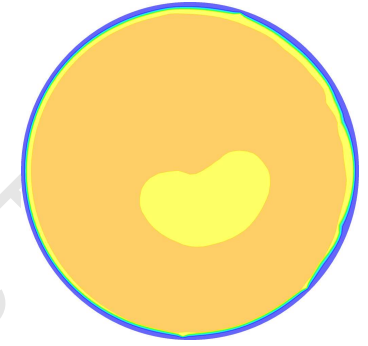

(f) $t o l$

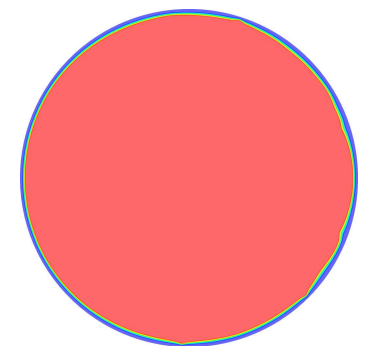

(i) $t o l$

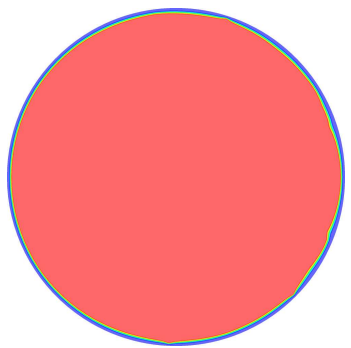

(l) tol

Temperature

$[\mathrm{K}]$

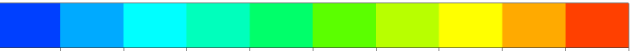

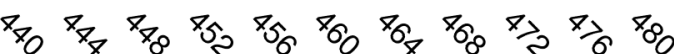

Figure 5.19: Temperature profiles for $M O N$ at $90^{\circ}$ ABDC for the motored case with air, iso-octane and toluene. From top to bottom: vertical mid-plane, horizontal plane near vale, horizontal mid-plane and horizontal plane nearest to piston. 
The asymmetrical temperature profiles are seen to diminish for the RON case. The strong vertical gradient is conserved under both RON and MON conditions. Under RON conditions, the vertical gradient is more pronounced and spans the majority of the cylinder height. The profiles for iso-octane and toluene are near identical. This is interesting since more toluene liquid was present up to this point, and hence it would be expected that the evaporative cooling associated with it would result in an obvious difference in the temperature distribution compared to iso - octane.

For the MON case, the vertical gradient is not as pronounced, with the majority of the incylinder mixture being at a uniform temperature. This is a result of the increased induction time and better development of swirling flow and turbulence within the cylinder under MON conditions.

Under RON conditions, the hotter gas is not located at the centre region of the combustion chamber, but rather at an outer region closer to the cylinder walls. This is in accordance with the findings by Liu et al. in [42]. In their study, a CFR engine was modified to act as an HCCI engine and run at $900 \mathrm{RPM}$ with a combustion wall temperature of $450 \mathrm{~K}$ and a mixture inlet temperature of $296 \mathrm{~K}$. Although these conditions differ from the current studies, the state of the in-cylinder mixture prior to compression is similar in that the bulk mixture temperature was lower than the combustion chamber wall temperature. Under RON conditions, the incylinder gas is at a lower temperature than the wall at the start of compression. This was also found in [42] and indicates that the gas adjacent the hot walls is heated by the walls during the first stages of compression, until it becomes hotter than the wall temperature because of compression-related temperature increase. This result that the fuel-air mixture adjacent the wall could be hotter than at the central regions, would imply that auto-ignition would initiate in these higher temperature regions.

The final temperature distributions are shown at $30^{\circ}$ BTDC in Figure 5.20 for RON and in Figure 5.21 for MON. 


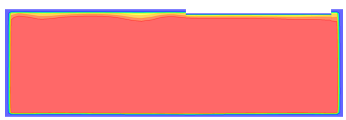

(a)

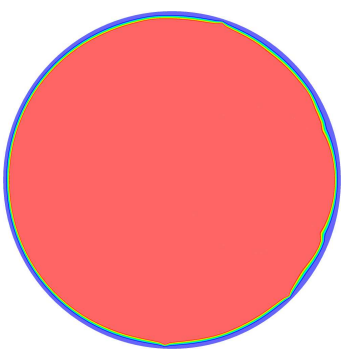

(d)

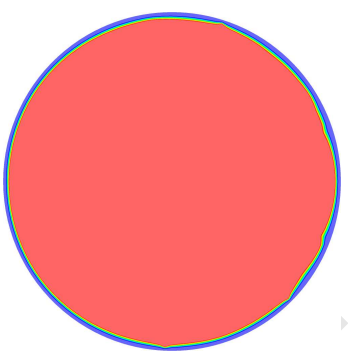

(g)

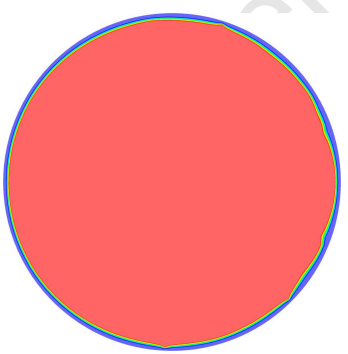

(j) air

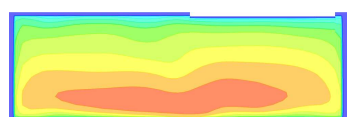

(b)

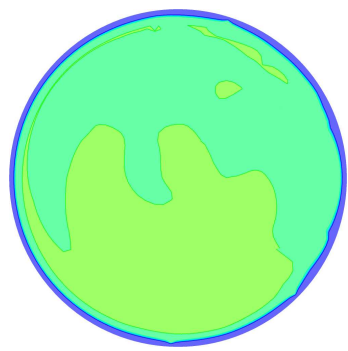

(e)

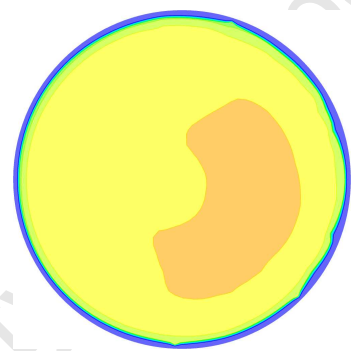

(h)

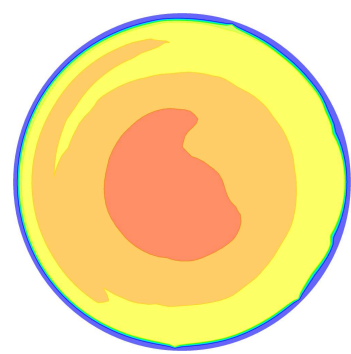

(k) $i$-oct

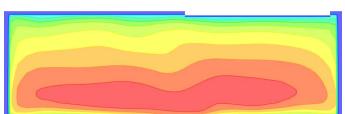

(c)

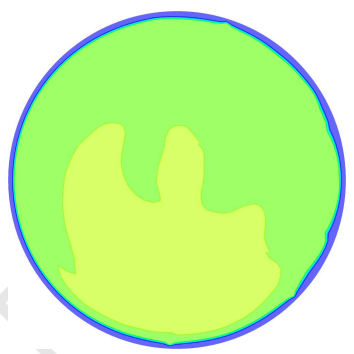

(f)

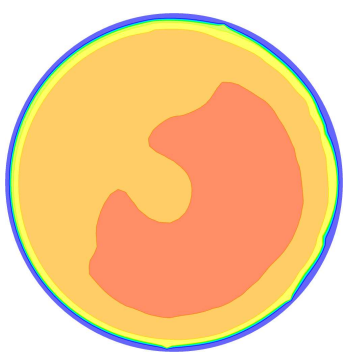

(i)

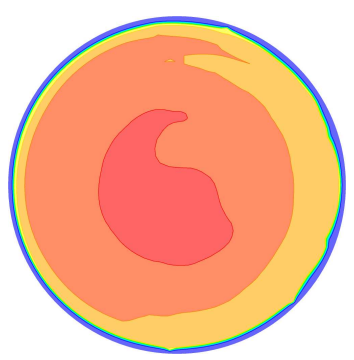

(l) tol

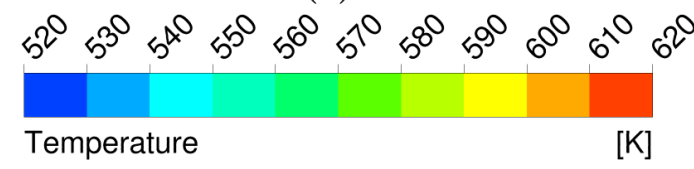

Figure 5.20: Temperature profiles for $R O N$ at $30^{\circ} B T D C$ for the motored case with air, iso-octane and toluene. From top to bottom: vertical mid-plane, horizontal plane near vale, horizontal mid-plane and horizontal plane nearest to piston. 


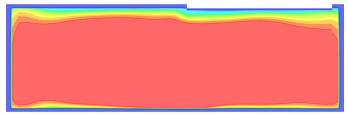

(a) air

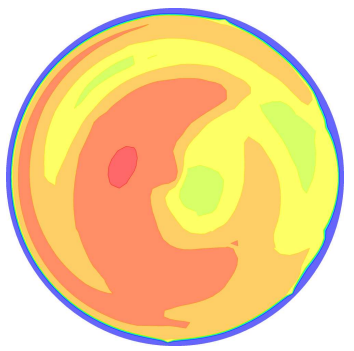

(d) air

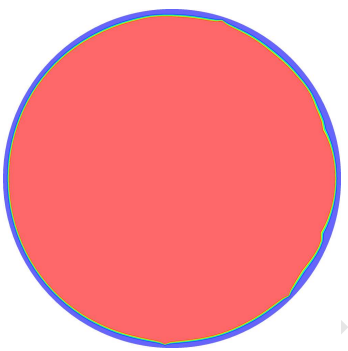

(g) air

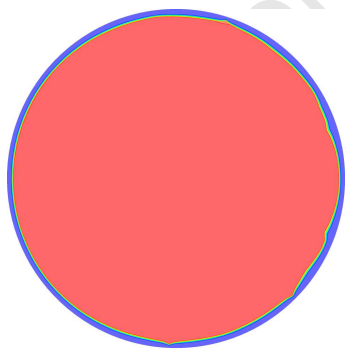

(j) air

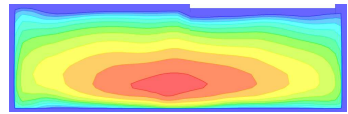

(b) $i$-oct

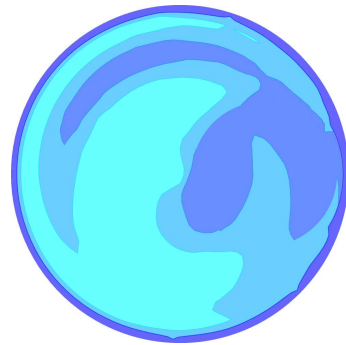

(e) i-oct

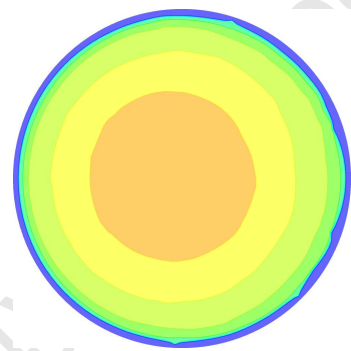

(h) $i$-oct

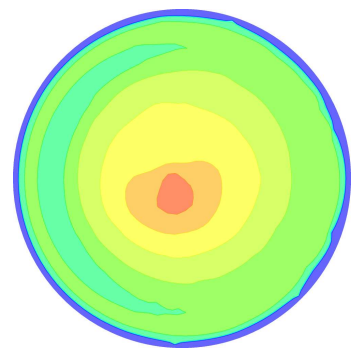

(k) i-oct

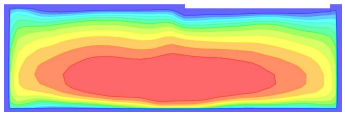

(c) tol

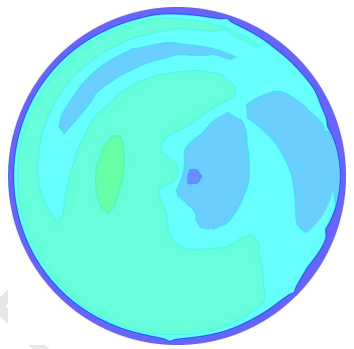

(f) tol

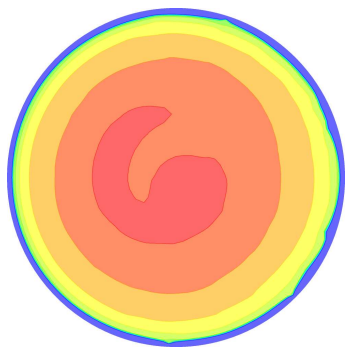

(i) tol

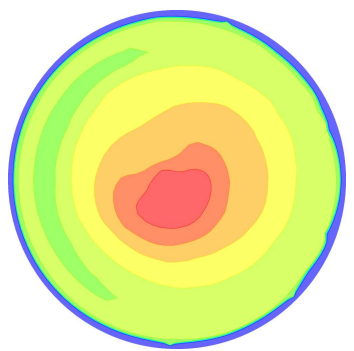

(l) $\mathrm{tol}$

Temperature

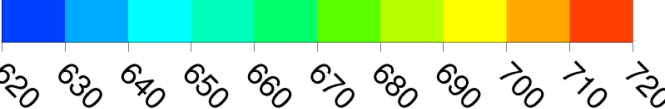

Figure 5.21: Temperature profiles for $M O N$ at $30^{\circ}$ BTDC for the motored case with air, iso-octane and toluene. From top to bottom: vertical mid-plane, horizontal plane near vale, horizontal mid-plane and horizontal plane nearest to piston. 
The vertical temperature gradient is conserved for the iso-octane-air and toluene-air mixtures under both RON and MON conditions. For the motored case however, the in-cylinder temperature distribution is uniform.

Where the in-cylinder temperature distribution is non-uniform, it is useful to know what proportion of the in-cylinder mass is at a certain temperature. Histograms showing the mass fraction distribution of temperature inside the combustion chamber for RON and MON - for motored air, iso-octane-air and tolune-air - are shown at IVC, $90^{\circ} \mathrm{BTDC}$ and $30^{\circ} \mathrm{BTDC}$ in Figure 5.22, Figure 5.23 and Figure 5.24 respectively.
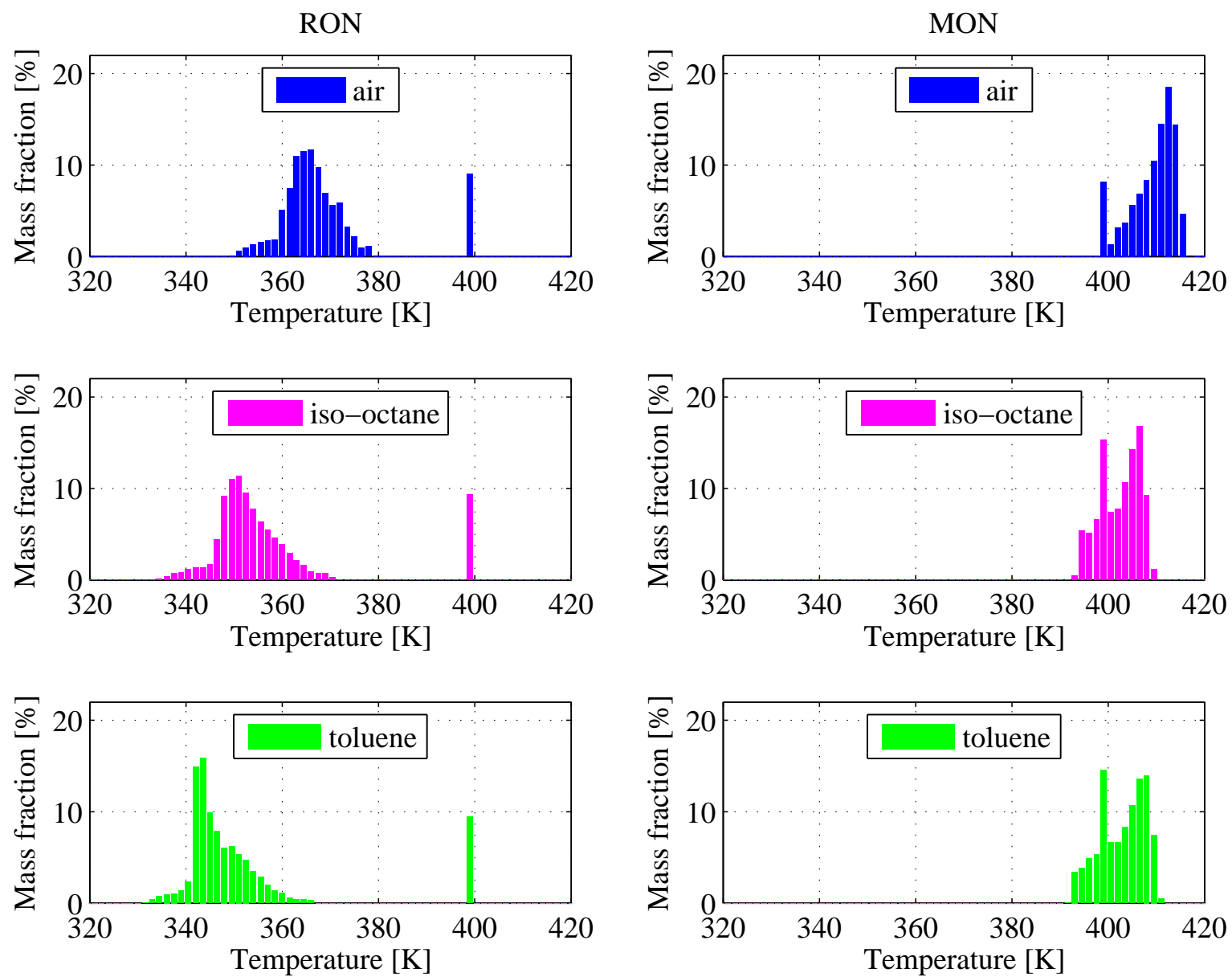

Figure 5.22: Histograms of mass fraction of unburnt gas at given temperatures at IVC. Bin width $=1.5 \mathrm{~K}$. 

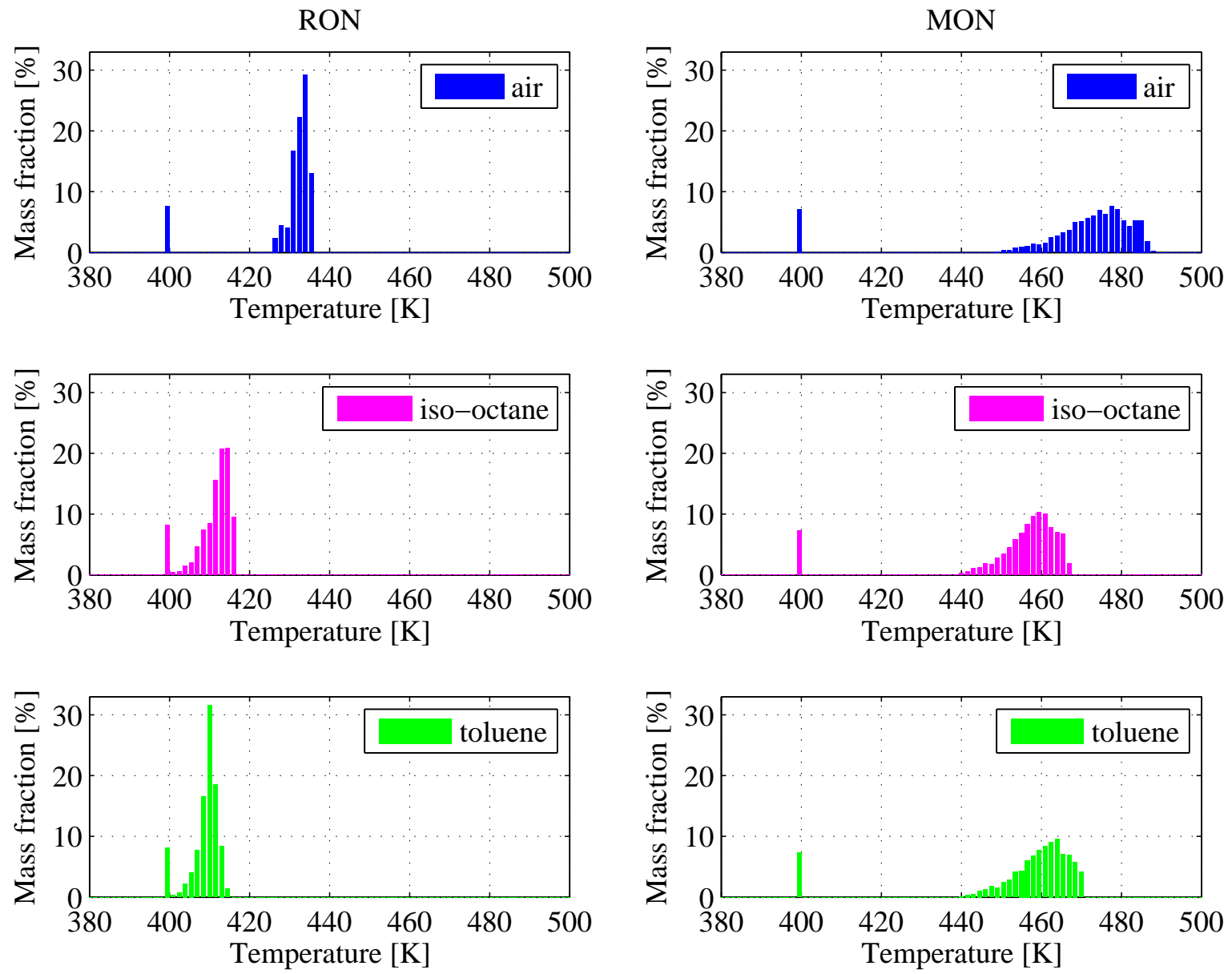

Figure 5.23: Histograms of mass fraction of unburnt gas at given temperatures at $90^{\circ} B T D C$. Bin width $=1.5 \mathrm{~K}$. 

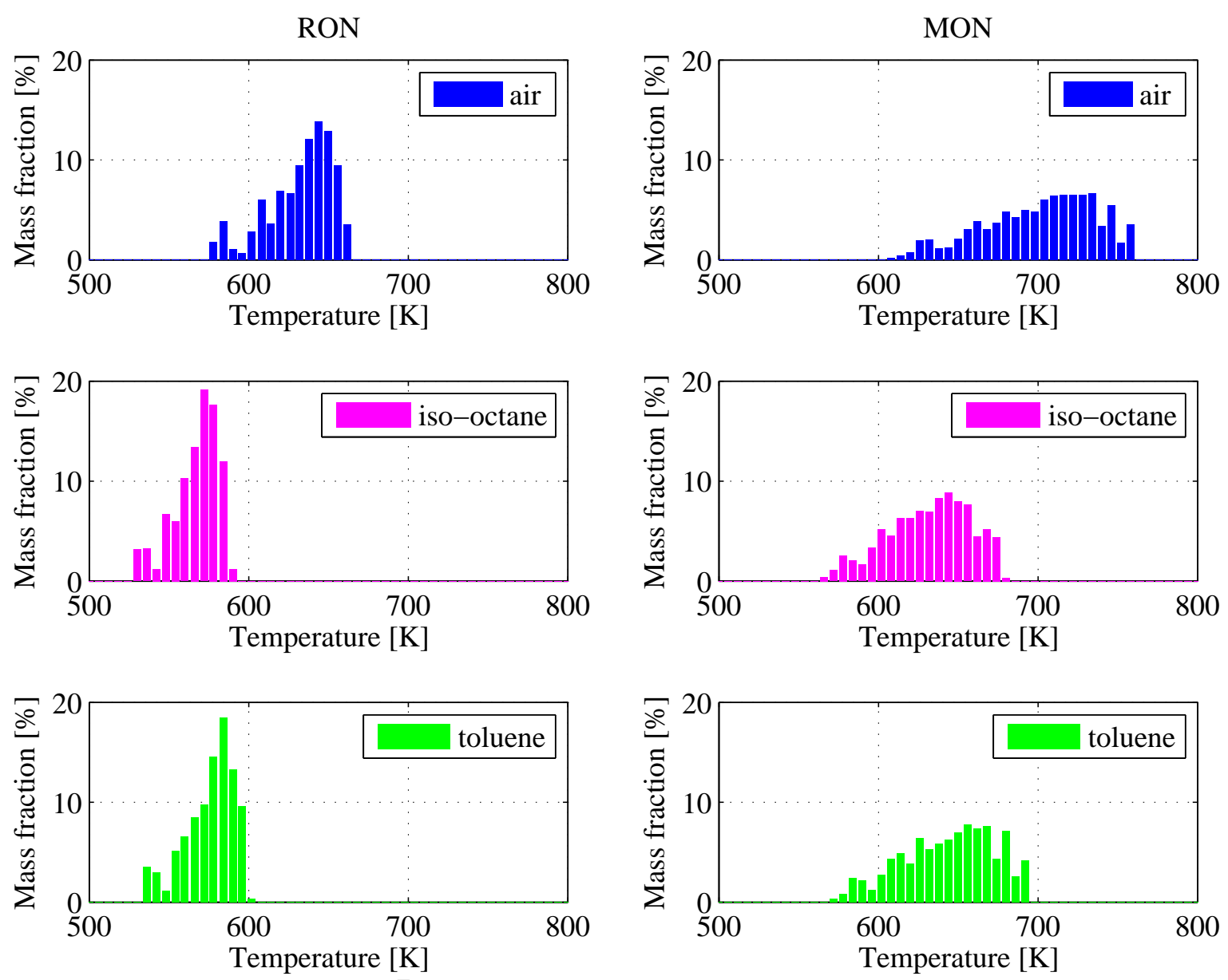

Figure 5.24: Histograms of mass fraction of unburnt mixture at given temperatures at $30^{\circ}$ BTDC. Bin width $=6 \mathrm{~K}$.

In Figure 5.22 and Figure 5.23 the lumped mass fraction of just less than $10 \%$ at $400 \mathrm{~K}$ represents the mass in the boundary layer adjacent the wall. This mass is still present at $30^{\circ}$ BTDC, but falls outside the temperature range presented in Figure 5.24. This should be seen as an exaggerated percentage. The reason for this could be the spacial discretization employed near the wall. This mass would reduce with finer grid resolution in this region. Nevertheless, these histograms are still particularly of interest as they could be used in multizone 0D models where one could adapt the cylinder zones to represent the distribution of temperature and mass from the results of the CFD analyses.

The average in-cylinder gas temperatures are summarized in Figure 5.25 . 


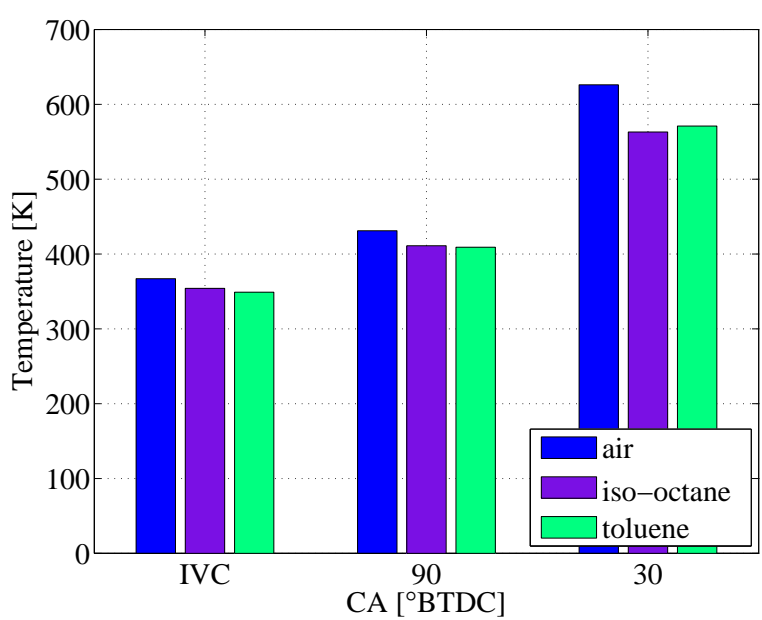

(a) $R O N$

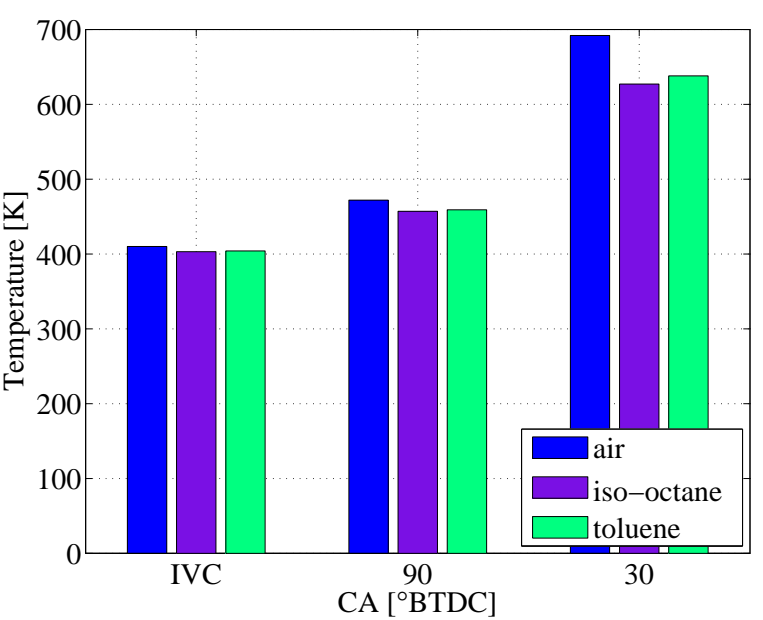

(b) $M O N$

Figure 5.25: Average in-cylinder gas temperature for different $C A$ during compression

The trends are very similar for RON and MON. This is interesting as the expectation was that the liquid fuel entering the port and cylinder in the RON case would result in different temperature trends. At IVC and $90^{\circ}$ BTDC the motored air temperatures are very similar to that of iso-octane-air and toluene-air mixtures. At $30^{\circ}$ BTDC the in-cylinder temperature for the motored case is significantly higher compared to the case where fuel was used - for both RON and MON. A rank change is observed between the $90^{\circ}$ BTDC and $30^{\circ}$ BTDC data sets. Even though the toluene-air mixture started off cooler in the RON case, it ends up hotter than the iso-octane-air mixture due to $\gamma$ effects on temperature during compression. The initial evaporative cooling benefit that the toluene provided is diminished during compression where $\gamma$ value differences come into play. This indicates that, when considering alcohol fuels with very high LHV, the $\gamma$ effects can be the dominating contributing factor to final mixture temperature.

\subsubsection{AFR}

Figure 5.26 shows the mass fraction of unburnt fuel-air mixture at a given $\lambda$ value within the combustion chamber at $30^{\circ}$ BTDC. This indicates the extent of mixing of the unburnt fuel-air mixture with the hot REG and also gives insight into the state of the fuel-air mixture in the cylinder. 

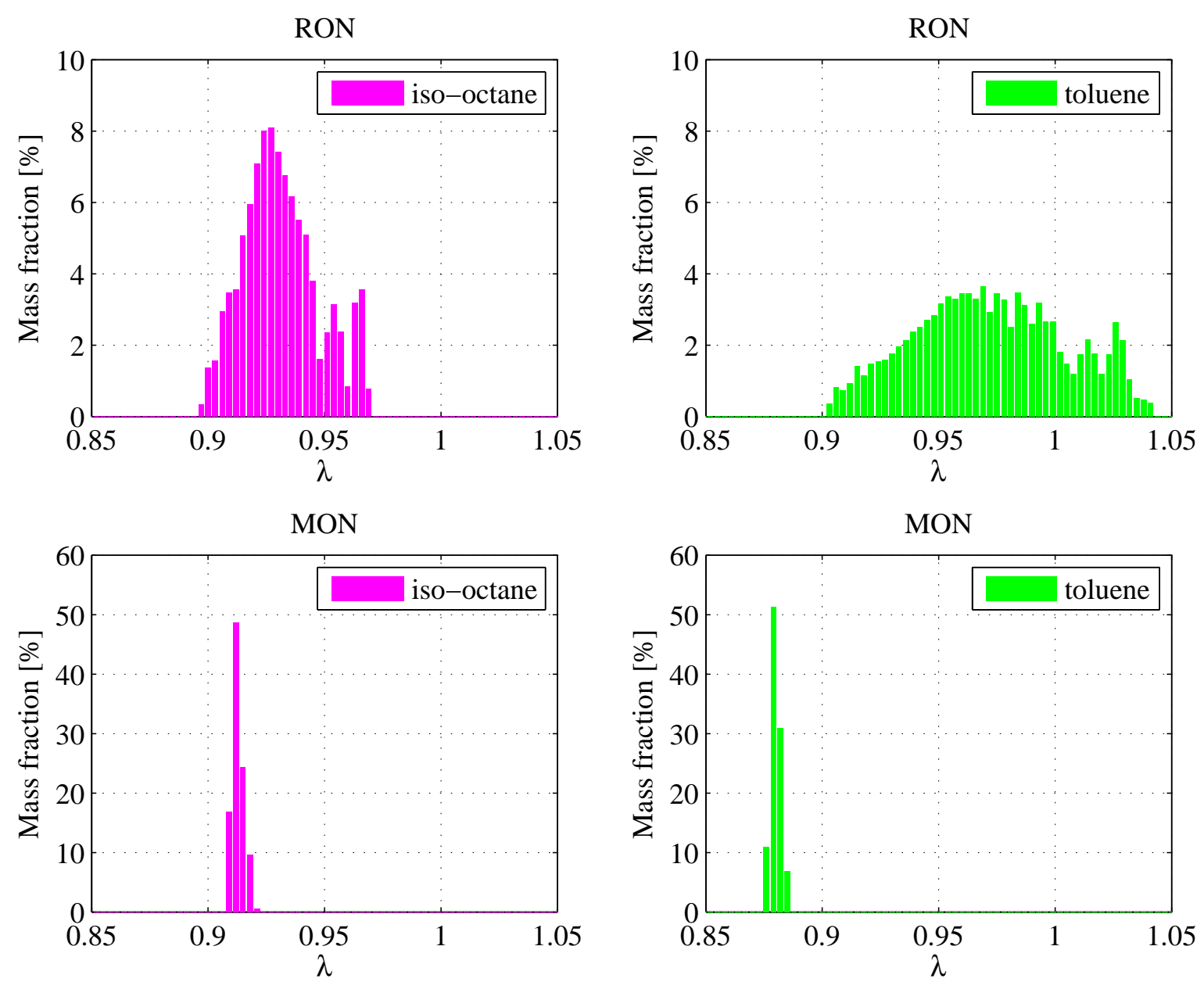

Figure 5.26: Mass fraction of unburnt fuel-air mixture at given $\lambda$ for $30^{\circ}$ BTDC. Bin width $=0.003$. 
For the MON case it is clear that the fuel-air charge mixes relatively uniformly with the hot REG, compared to RON. The variable $\lambda$ in the RON can be attributed to the fact that all the liquid fuel was not fully evaporated before it reached the cylinder. The higher engine speed of the MON test results in faster inlet flows. However, the fuel spray travels approximately 3 times further than it does under RON conditions, due to the extra heating manifold section. This increased intake time and more complete evaporation ensures a more homogeneous charge entering the cylinder. The mass-averaged in-cylinder $\lambda$ values at $30^{\circ} \mathrm{BTDC}$ are presented in Table 5.3 .

Table 5.3: Average $\lambda$ at $30^{\circ} B T D C$

\begin{tabular}{lcc}
\hline & RON & MON \\
\hline iso-octane & 0.938 & 0.915 \\
toluene & 0.982 & 0.882
\end{tabular}

The in-cylinder profiles of $\lambda$ on a plane through the cylinder as shown in Figure 5.27 are shown in Figure 5.28 for RON tests.

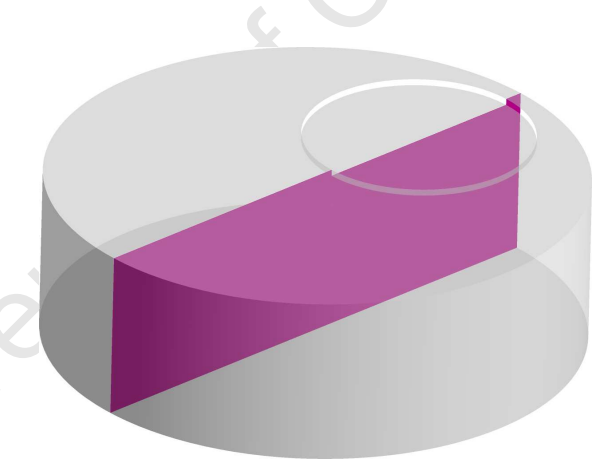

Figure 5.27: Cylinder mid-plane at $30^{\circ} B T D C$ where profiles of $\lambda$ are shown

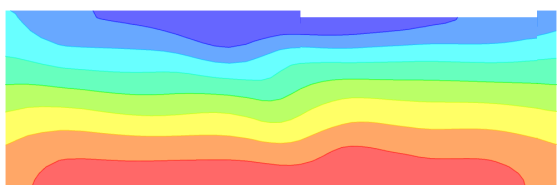

(a) iso-octane

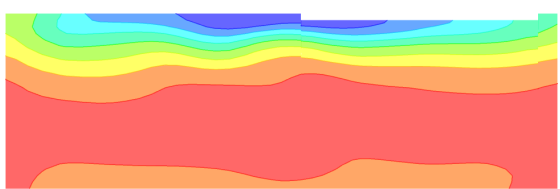

(b) toluene

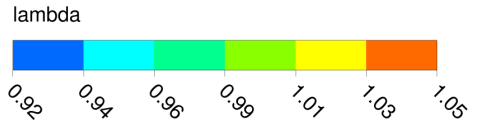

Figure 5.28: $\lambda$ at $30^{\circ} B T D C$ for $R O N$ tests

A vertical gradient of $\lambda$ is observed indicating further that fuel-air mixing did not occur uniformly, with $\lambda$ ranging from 0.92 to 1.05. Similar profiles of $\lambda$ are shown for the MON 
tests in Figure 5.29. For the toluene-air mixture under RON conditions the vertical gradient is slightly richer at the piston interface, lean in the centre and then richer again closer to the cylinder top. The slightly richer mixture right above the piston top can be explained by considering that more toluene-liquid survives in-cylinder and is thus pulled down to the bottom, where it evaporates during compression, thus causing a slightly richer mixture above the piston.

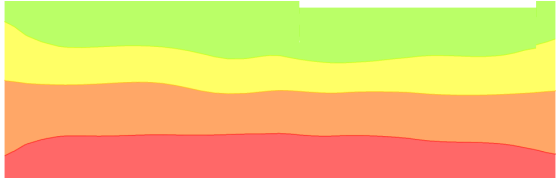

(a) iso-octane

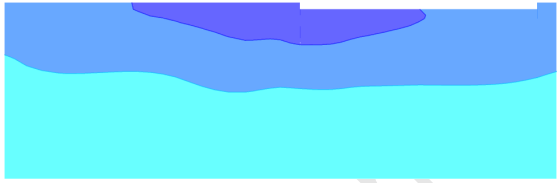

(b) toluene

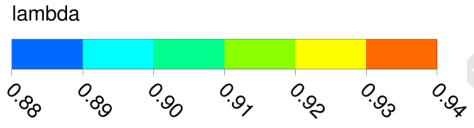

Figure 5.29: $\lambda$ at $30^{\circ} B T D C$ for $M O N$ tests

It can be seen that mixing occurs more uniformly for MON, with $\lambda$ ranging from 0.88 to 0.94 . For MON with toluene, this range is only from 0.92 to 0.94 . The vertical gradient is also less pronounced. Under both RON and MON conditions, the vertical gradient of $\lambda$ is still evident. For both RON and MON the profiles are symmetrical about the cylinder axis. 


\section{Discussion of results}

This chapter gives a final discussion of the results presented in the previous chapter.

\subsection{Evaporative cooling}

Under MON conditions, it was found that no liquid fuel reached the port or cylinder, and thus evaporative cooling did not come into play. Since the mixture temperature is controlled at the port, the lack of unevaporated droplets at this stage means that the LHV of the fuel cannot affect the fuel's performance in the test. The LHV is completely compensated for by the power put into the mixture heating element controlling the mixture temperature. Under RON conditions, both toluene and iso-octane liquid fuel were present in the port and cylinder, and more toluene liquid fuel was present in the cylinder compared to iso-octane. Since toluene has a higher LHV - 34\% higher in fact - than iso-octane, and since more toluene fuel entered the cylinder as liquid, a greater evaporative cooling affect was exhibited with toluene.

This was put into context by calculating the maximum potential cooling capacity of the liquid in the port and cylinder. In the port volume, this cooling capacity was calculated to be about $87 \mathrm{~K}$ for toluene and only $28 \mathrm{~K}$ for iso-octane. This is more than 3 times greater than that calculated for iso-octane. It should be noted, however, that the toluene RON simulations were run slightly leaner than the iso-octane ones $(\lambda=0.98$ for toluene vs. $\lambda=0.94$ for isooctane). If toluene was run at a similar $\lambda$ value to iso-octane, more liquid mass and hence a greater cooling capacity would have resulted.

Based on these calculations, if the majority of the energy of vaporisation were sourced from the gas-phase, it would be expected that the toluene-air mixture would end up at much lower temperatures than the iso-octane-air mixture. However, the average in-cylinder toluene-air mixture temperature at IVC was found to be only about $10 \mathrm{~K}$ cooler than the iso-octaneair mixture. This indicated that a large amount of the LHV was sourced from the hotter combustion chamber walls, as opposed to the surrounding gas. 
What was interesting was that, even though the toluene-air-mixture was cooler than the iso-octane-air mixture at IVC, it became hotter during compression and, at $30^{\circ} \mathrm{BTDC}$, the toluene-air mixture was about $10 \mathrm{~K}$ hotter than the iso-octane-air mixture. This would suggest that the compression heating behaviour of the fuel-air mixture constituents, i.e. the value of the ratio of specific heats $(\gamma)$, diminish the evaporative cooling benefit seen with toluene compared to iso - octane, since toluene - air $\gamma$ values are greater than that for iso-octane-air. Under RON conditions, the $\gamma$ values of toluene-air were about $1.5 \%$ greater than that for iso - octane -air over the compression stroke (see Figure 5.11). However, this translates to $4 \%$ in the adiabatic compressive heating effect, since compressed temperature is proportional to the compression ratio to the power of gamma minus one, as shown below:

$$
\frac{T_{2}}{T_{1}}=\left(\frac{V 1}{V 2}\right)^{\gamma-1}
$$

This seemingly small difference in $\gamma$ thus becomes significant in the temperature development during compression.

From the point of flame initiation, the flame front would continue to compress the unburnt gas adiabatically. The effect that the difference in $\gamma$ has on compression heating is thereby even further increased. Furthermore, $\gamma$ decreases with increasing temperature, however as seen in Figure 5.11, the difference in $\gamma$ values for iso-octane and toluene did not decrease significantly as temperature increased. This indicates that the difference in compressive heating effect between the two mixtures became greater as compression progresses.

This finding is in stark contrast to that found by Moran in [7, 8], where it was concluded that fuel evaporative cooling can almost fully explain fuel octane sensitivity.

\subsection{In-cylinder temperature distribution}

One of the main drivers for the current work was to provide a better estimate of the shape and size of the temperature profile setup in the CFR engine at IVC. Knowing the temperature profile and where the hottest regions are within the cylinder volume allows for better prediction of the location where auto-ignition would initiate.

In [21], a multi-zone 0D thermodynamic CFR engine model was developed in order to study the effects of such a temperature profile. Certain assumptions regarding the shape and size of the temperature profile were made:

1. The temperature profile varies radially from the cylinder axis to the wall.

2. This temperature profile is symmetrical about the cylinder axis. 
3. The temperature profile does not vary axially along the cylinder.

Since information about the shape of the profiles was not available, a range of 6 profiles were studied: three where the chamber wall temperature is hotter than the mixture temperature ((a) to (c) in Figure 6.1), and three where it is cooler than the mixture temperature ((d) to (f) in Figure 6.1).
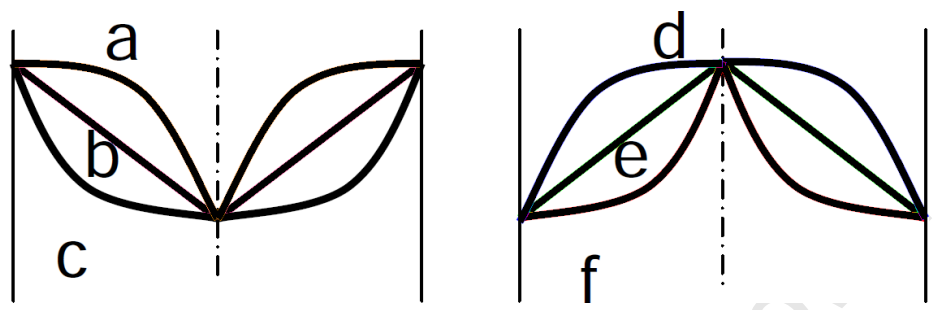

Figure 6.1: Assumed initial radial temperature profiles use in [21]

In the current work, it was found that there is a strong axial temperature gradient for both RON and MON. Furthermore, under RON conditions, the radial temperature profiles were not found to be symmetrical about the cylinder axis. For the sake of comparison with that assumed in [21, the temperature profiles along 6 different radial paths are shown at IVC for iso-octane-air and toluene-air in Figure 6.3 for RON and in Figure 6.4 for MON. The radial paths coincide with the planes that were used to present the temperature contours in Section 5.2 .2 and is indicated in Figure 6.2 .
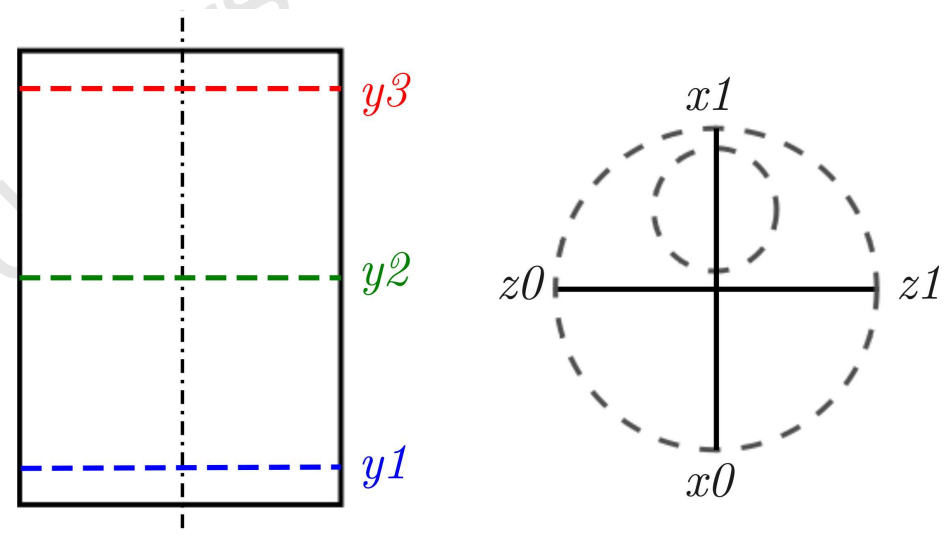

Figure 6.2: Diagram showing lines along which temperature profiles are plotted 

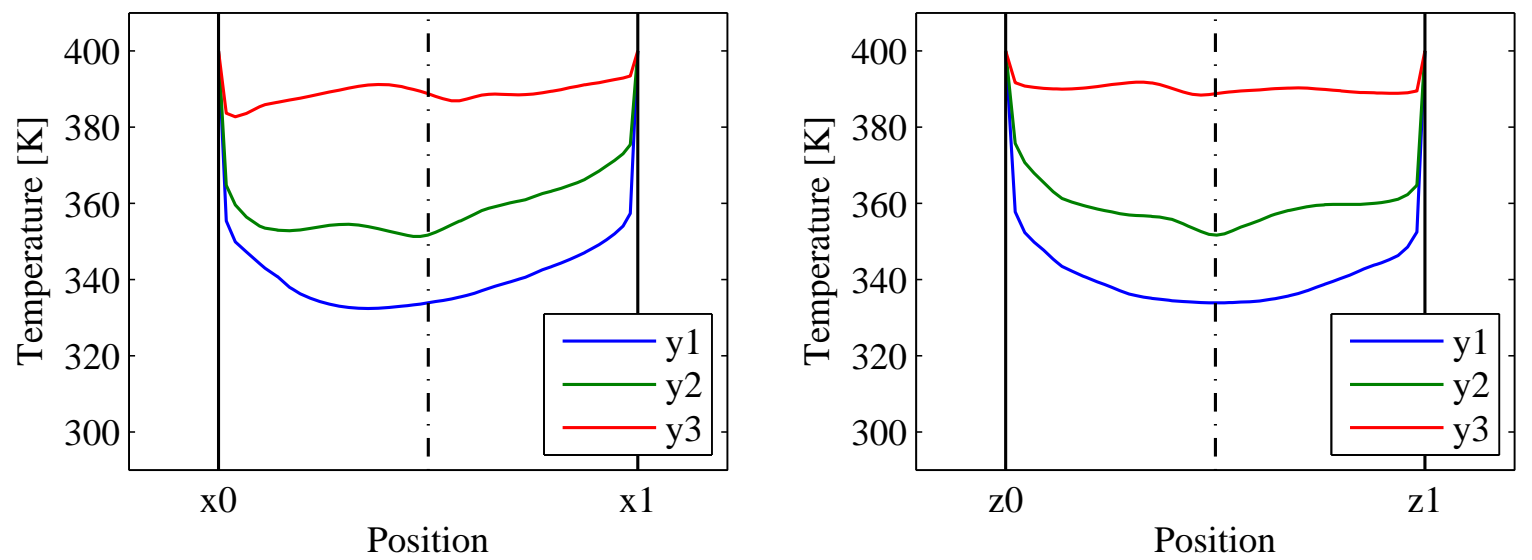

(a) iso-octane
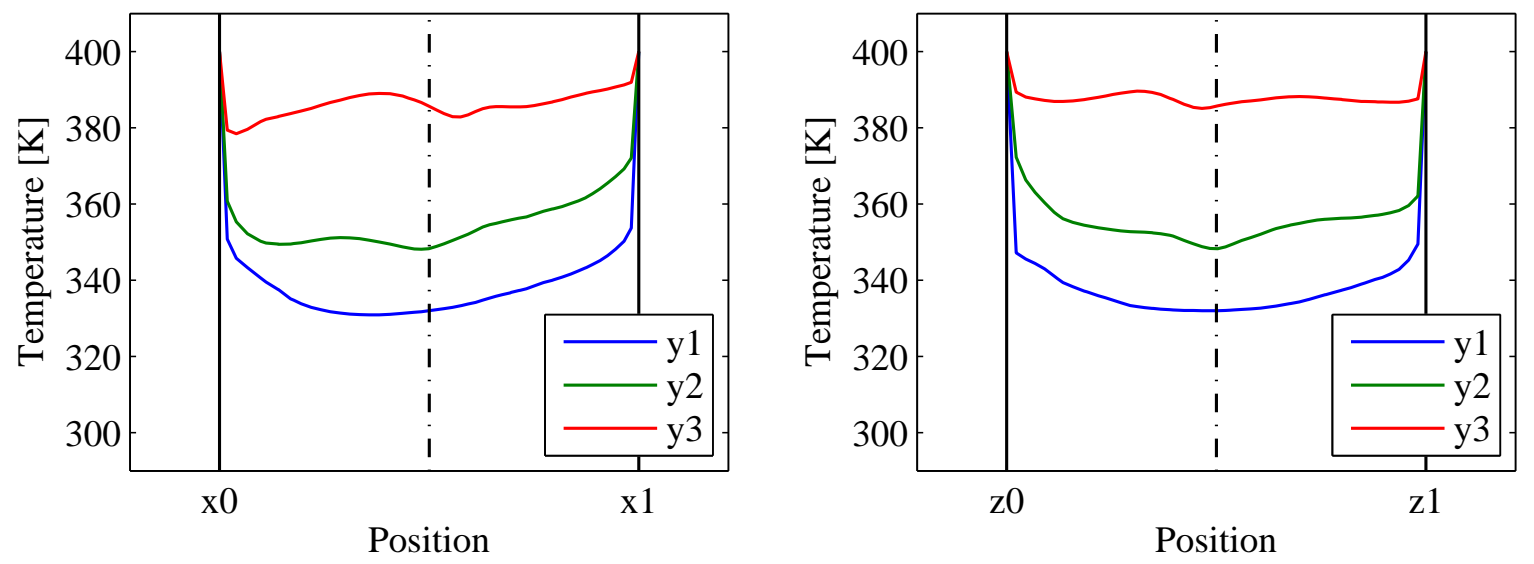

(b) toluene

Figure 6.3: Radial temperature profiles at 3 different positions along the cylinder axis at IVC for RON tests 

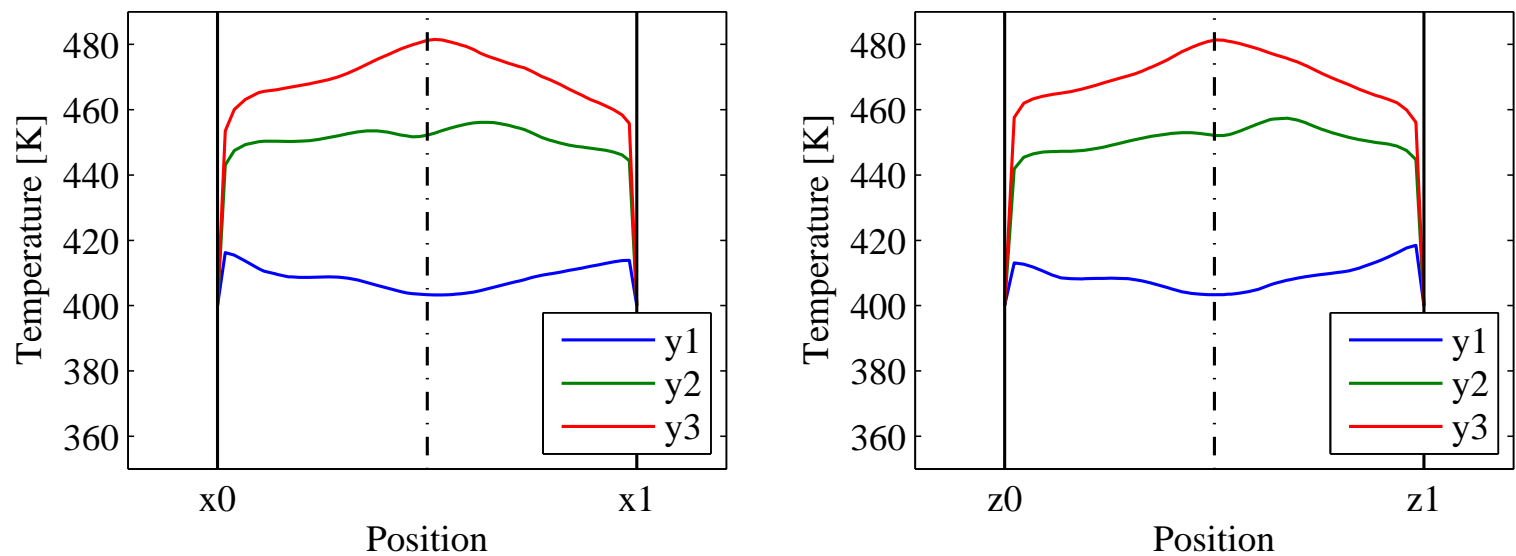

(a) iso-octane
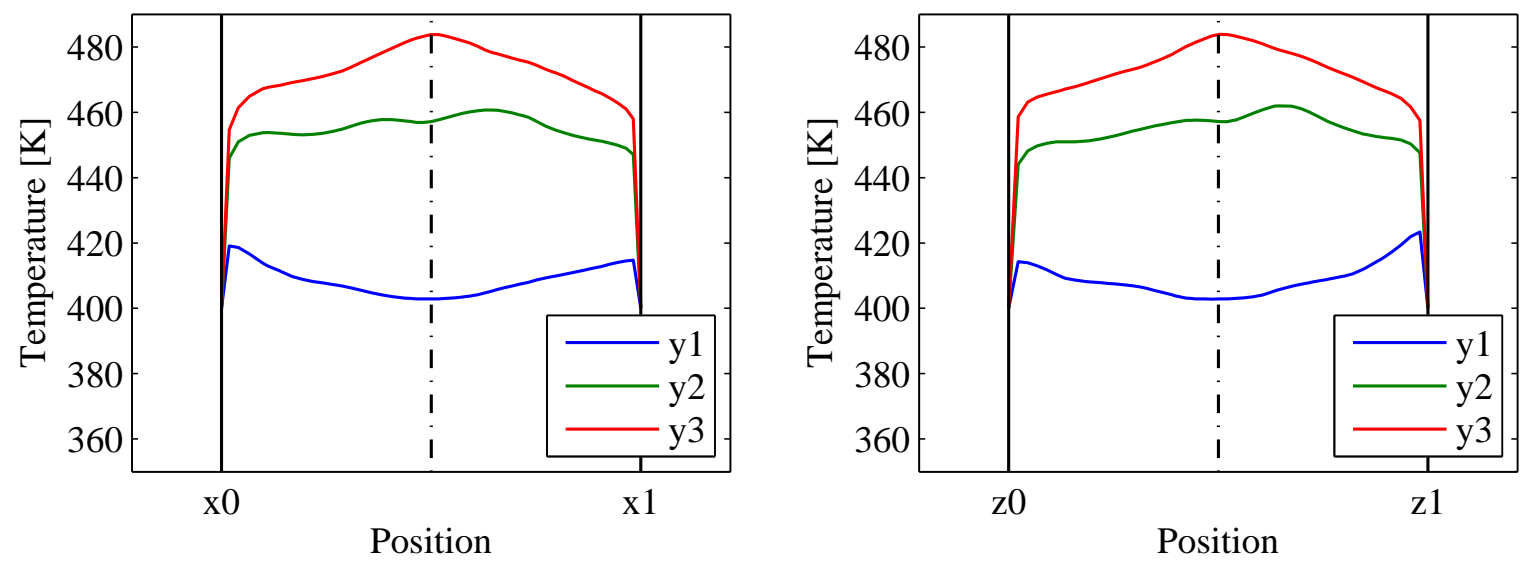

(b) toluene

Figure 6.4: Radial temperature profiles at 3 different positions along the cylinder axis at IVC for MON tests

For the RON case, the temperature profiles were not symmetrical about the cylinder axis. For both the RON and the MON cases, the temperature profiles changed with axial height. Since this strong axial temperature gradient was not obvious and not assumed in literature, it requires further investigation.

The asymmetry observed under RON conditions for iso-octane-air and toluene-air was also observed for the motored RON case, implying that it was not only a result of the evaporative cooling, but also the lower engine speeds associated with the RON tests. The asymmetry can be attributed to a combination of the following:

1. Evaporative cooling of the gas phase due to the evaporation of the liquid fuel entering the cylinder, as well as the liquid fuel already present in the cylinder. 
2. The lower speeds associated with the RON setup. Under RON conditions, the lower engine speeds resulted in less developed in-cylinder swirling flow and turbulence, compared to the MON tests. This resulted in poorer mixing of the fresh charge with the REG, which, in turn, resulted in less homogeneous distribution of in-cylinder properties, compared to MON.

This asymmetrical temperature distribution was not exhibited for the MON case. The higher speeds allowed better establishment of cylindrical swirling flow and turbulence, which helped the fresh charge and exhaust residuals to mix. This had implications on the thermal and AFR distributions within the cylinder. This was confirmed when looking at the AFR distribution in the cylinder at $30^{\circ} \mathrm{BTDC}$, and was also noted in the literature [40].

\subsection{Combustion chamber wall temperatures}

The same chamber wall temperature BC of $400 \mathrm{~K}$ was used for all simulations. This chamber wall temperature had a very different effect on the shape of the temperature profile produced, depending on the temperature of the in-cylinder gas. When the chamber wall temperature was hotter than the initial gas temperature prior to compression, the wall heated the adjacent gas, and this gas would then be further heated with the rest of the gas during compression. This would result in an intermediate temperature profile as shown in Figure 6.5.

\section{Hotter gas}
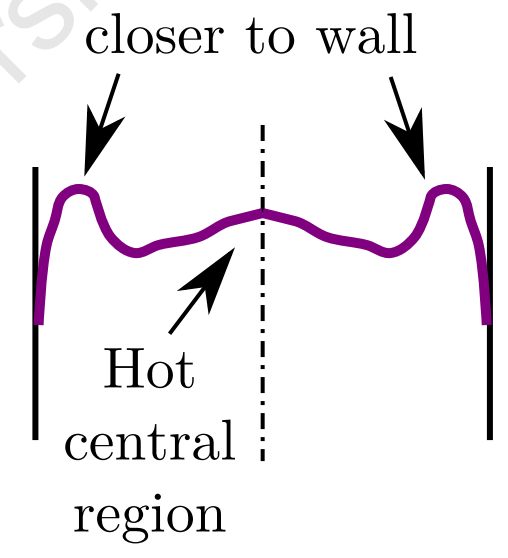

Figure 6.5: Radial temperature profile which results where the chamber wall temperatures are higher than the initial in-cylinder gas temperature. This temperature profile would be exhibited at some point during compression when the gas temperature has risen above the wall temperature.

The temperature distribution at this intermediate time during compression does not have the hottest gas at the centre regions, but rather closer to the walls. This was observed in the current work under RON conditions and also found in [42]. 
This effect did not come into play under MON condition. However, the chamber wall BC of $400 \mathrm{~K}$ was chosen to be an average between the coolant temperature of $373 \mathrm{~K}$ and the experimentally measured wall temperature in [12] of $423 \mathrm{~K}$. In Figure 5.22 it was seen that all of the in-cylinder gas is below $420 \mathrm{~K}$ for both fuel types, as well as the motored case. In fact, for iso-octane-air and toluene-air, some of the mass was actually below $400 \mathrm{~K}$. This means that, if the wall temperature was chosen as the higher extreme of $423 \mathrm{~K}$, the wall heating affect would have come into play even in the MON tests. The higher temperatures associated with the MON tests would also make the possibility of higher combustion chamber wall temperatures plausible. This has implications on the prediction of knock, as auto-ignition will initiate in hotter regions within the cylinder.

\subsection{The effect of the choice of fixed surface temperature bound- ary conditions}

The above discussed results should be understood in the context of the limitations of the current work. The surface temperatures of the manifold, port and cylinder walls were assumed to remain constant. This is certainly no the case for a motored versus a fired operation. The purpose of the motored simulations in the current work was not to accurately model the engine under cool motored conditions, but rather to illustrate the effect of th fuel addition to the thermodynamic conditions development. Thus the same boundary conditions must have been used in order to make the comparison.

Moreover, if latent heat is in fact derived primarily from the engine surfaces, these surfaces should cool down, and the extent of cooling will be dependent on the fuel used. However, in order to model this properly, a FEM analysis of the wall heat transfer would have to be added, including the material properties of the wall and ideally the coolant flow dynamics. The model would also have to be run over over many cycles, which was not practical given the time taken for a single cycle to be simulated.

Furthermore, it would have been useful to measure the actual outer wall temperatures of the manifold walls experimentally. This was however not done and is a clear weakness of the current work. 


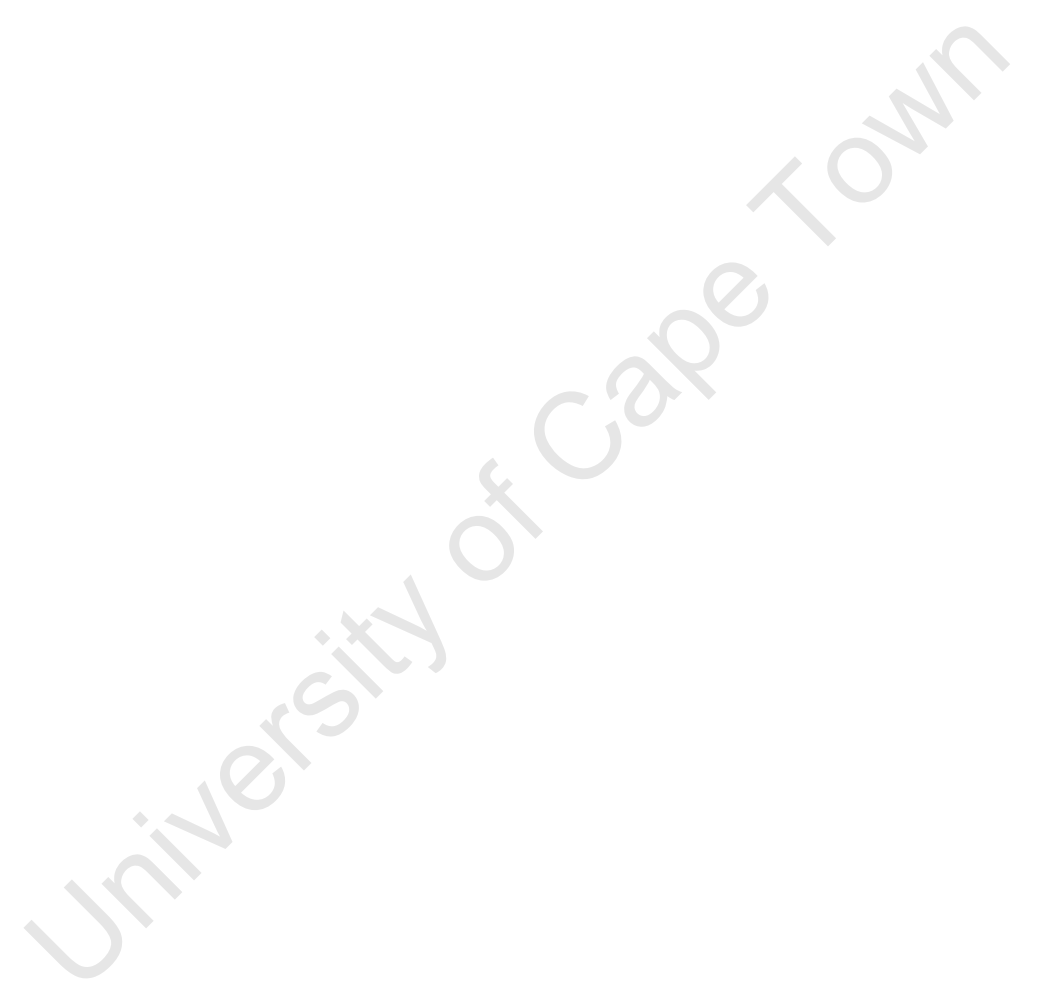




\section{Conclusions}

Based on the results presented and discussed in the previous chapters, the following conclusions could be made.

A 3D model of the intake and compression cycle of the CFR engine was developed in order to study the thermal gradient and heat transfer which occurs for both RON and MON test conditions. The model with all its submodels worked and has provided valuable insight in a number of areas.

Under MON conditions, all the liquid fuel entered the port and cylinder pre-evaporated and hence did not produce evaporative cooling in the cylinder. Under RON conditions, a substantial amount of the fuel entered the port as liquid, and a small amount - up to $3 \%$ iso-octane and 5\% toluene - entered the cylinder as liquid.

The maximum cooling potential of the liquid in the cylinder was found to be negligible. The maximum cooling potential of the liquid fuel in the intake port - $28 \mathrm{~K}$ for iso-octane, and $86 \mathrm{~K}$ for toluene - was substantial. This however only translated to the in-cylinder toluene-air mixture being $10 \mathrm{~K}$ cooler than that of the iso-octane-air mixture at IVC. A large amount of the LHV required to evaporate the fuel was thus not sourced from the surrounding gas, but rather the hotter chamber surfaces. This shows heat transfer to be a significant factor, since it largely smudged out the differences in fuel LHV effect on in-cylinder temperatures.

A clear in-cylinder temperature distribution was observed for both test fuels and the motored case under both RON and MON conditions. Strong axial temperature gradients were observed. The radial temperature profile shapes were greatly influence by the temperature differential between the chamber walls and gas within it. The higher engine speeds associated with the MON tests resulted in more turbulence and better developed swirling flow within the cylinder, compared to RON. For RON, this resulted in asymmetry of the temperature profiles in the RON tests. 
For both RON and MON tests, the compression heating behaviour of the fuel-air mixture constituents, i. e. $\gamma$, had a significant influence on the final in-cylinder gas temperatures. This was in contrast with the weak effect that LHV had on the start of compression temperatures. It can thus be said that $\gamma$ effects during compression have a much bigger influence on end-gas temperature than evaporative cooling effects during intake. 


\section{Recommendations}

Based on the work done in this thesis, together with the conclusions that could be drawn from it, various recommendations regarding future research can be made.

\subsection{Further CFD modelling with the existing model}

The CFD model developed could be extended to further investigate some of the important findings, as listed below.

1. Grid sensitivity studies, or rather grid convergence studies, should be undertaken to determine the level of accuracy of the results found in this work. This could be done by rerunning one test condition and fuel with a mesh consisting of double the amount of cells. Furthermore error analysis based on [62] could be conducted to add numerical error values to the grids used.

2. Sensitivity studies on both the wall temperature values and the hot REG temperature values should 00be conducted. Since heat transfer effects have been shown to play a significant role in the in - cylinder temperature distributions, sensitivity studies such as these would add further value.

3. Testing of other single component fuels with differing thermodynamic properties, such as methanol, $n$-heptane and hexene, and testing of multi-component fuels, such as PRF blends, would be useful to gain further insight into OS and fuel effects during octane rating.

\subsection{Simple 0D and 1D engine modelling to investigate $\gamma$ effects}

The effect of $\gamma$ values on piston and flame compression heating can readily be done using a $0 \mathrm{D}$ or $1 \mathrm{D}$ engine model. These would require a fraction of the computational cost and time compared to full 3D CFD. The use of such a simple model would make it possible to investigate this $\gamma$ effect for all the major hydrocarbon structure classes, and various other fuel compositions. 


\subsection{Improvement of the results obtained from CFD analyses}

Minor improvements in the existing model could result in further valuable insight. These improvements should reduce the need for assumptions regarding the initial and boundary conditions which were chosen. Possible improvements are suggested below:

1. Inclusion of the exhaust valve and port would allow for the exhaust flow processed to be accounted for in the model. This would remove the need to patch end-of-exhaust REG conditions - such as temperature, pressure and composition - onto the computational domain.

2. Once (1) is implemented, the CFD calculation could be run over many cycles, and the flow in the cylinder due to the inlet and exhaust processes could be fully developed. Such a study should be run over many cycles due to the highly transient cycle-to-cycle variations. Cycle averaging over many cycles would result in more representative results. The latter would also make it possible to adjust the MON heater element temperature based on cycle-averaged values and also allow for stabilisation of conditions to give better start of simulation gas boundary conditions.

3. A comprehensive 1D engine model could also be used to obtain better gas BCs.

\subsection{Experimental work required to provide better initial and boundary conditions}

More experimental work is required to obtain better initial and boundary conditions for future engine modelling. For CFR engine models, more information is required to characterize the fuel spray, in particular initial droplet sizes and distributions. This could be attempted by:

1. incorporating optical access windows into the manifold and port sections to validate the distance travelled by the droplet spray prior to evaporation;

2. using experimental particle sizing techniques such as PDPA or a Malvern particle sizing device.

The combustion chamber wall temperatures, which have been shown to be important, could be further investigated since these can influence the location of auto-ignition significantly. Although wall temperature sensitivity tests can provide further insight into this, experimental investigation could be useful. Such investigations could be done by:

1. incorporating in-cylinder surface thermocouples; 
2. measurement of cooling water inlet and outlet temperatures, combined with a CFD model of the water cooling channels.

The study has also shown that the biggest thermal changes occur in the inlet and port. An experimental investigations of the thermal behaviour in the inlet and port an thus add useful insight. Comparison of such experimental work with the current work would also be useful in proving, disproving some of the findings made in the current work.

\subsection{Combustion and auto-ignition modelling using full 3D CFD}

Full 3D CFD of the combustion processes would provide a superior method for studying flame front propagation, heat release and composition change, as well as flame front compression of the unburnt gas during combustion. CFD could also be used to model auto-ignition of the end gas, taking into account the thermal and composition distribution within the cylinder.

At this stage, however, the computational cost associated with accurate and extensive CFD combustion modelling is still a limiting factor, since many simplifying assumptions need to be made to reduce modelling complexity. Studies such as suggested previously in this chapter may thus be better options of future work until new research emerges, or improved computational technologies are implemented. 


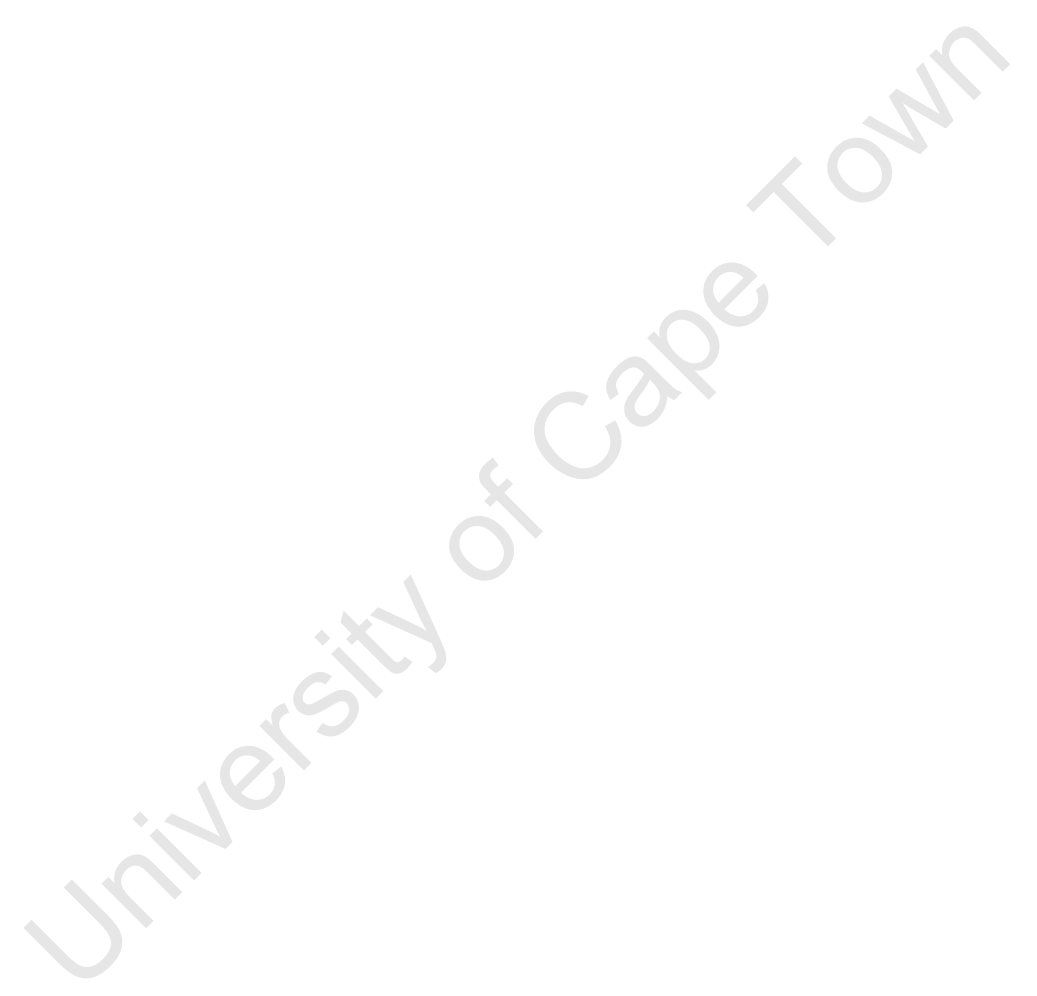




\section{A. User-defined functions}

\section{A.1 DPM mass flow rate UDF}

A UDF to regulate the mass flow rate of the fuel spray injection was developed. The mass flow rate for the liquid fuel injection was calculated such that it would ensure a stoichiometric AFR of the mixture at the end of compression.

The main steps followed in the UDF are listed below:

1. Initialize variables

2. Get the mass flow rate of air though the injection plane

3. Calculate the required mass flow rate of liquid fuel

4. Loop over all particle streams in an injection and assign to it the required mass flow rate

The fuel injection mass flow rate was calculated as:

$$
\dot{m}_{f u e l}=\frac{\dot{m}_{\text {air }}}{A F R_{\text {stoic }}}
$$

The mass flow rate of air, $\dot{m}_{\text {air }}$, used in this calculation was calculated across the plane where the spray was injected.

For iso-octane fuel spray, the UDF is given as: 


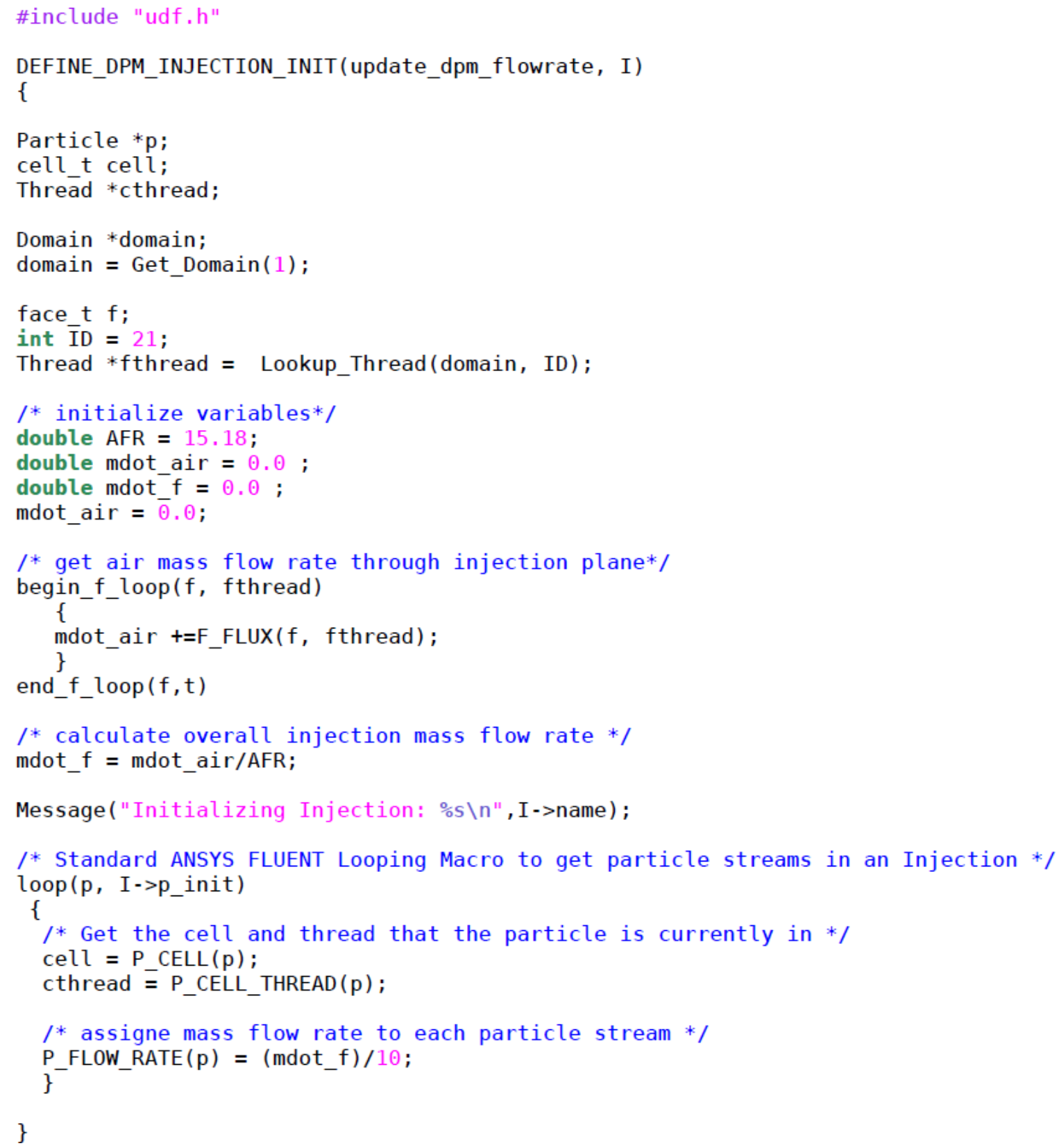




\section{References}

[1] J. B. Heywood. Internal combustion engine fundamentals. McGraw-Hill, international edition, 1988. Chapter 9.

[2] V. Mittal, J. B. Heywood, and W. H. Green. The underlying physics and chemistry behind fuel sensitivity. SAE International, 2010. SAE paper 2010-01-00617.

[3] American Society for Testing and Materials. Standard test method for knock characteristics of motor fuels by the research method, 1983.

[4] American Society for Testing and Materials. Standard test method for knock characteristics of motor and aviation fuels by the motor method, 1983.

[5] Test methods for rating motor, diesel and aviation fuels, volume 05.04. American Society for Testing and Materials, 1984.

[6] A. Swarts and A. D. B. Yates. In-cylinder fuel evaporation and heat transfer information inferred from the polytropic character of the compression stroke in a spark ignition engine. SAE International, 2004. SAE paper 2004-01-1856.

[7] D. P. Moran and A. B. Taylor. An evaporative and engine-cycle model for fuel octane sensitivity prediction. SAE international, (SAE paper 952524), 1995.

[8] D. P. Moran and A. D. B. Yates. The effects of fuel evaporation on engine knock. Master's thesis, University of Cape Town, June 1993.

[9] M. Mehl, T. Faravelli, and E. Ranzi. Experimental and kinetic modelling study of octane number and sensitivity of hydrocarbon mixtures. SAE International, 2005. SAE paper 2005-24-077.

[10] M. Mehl, T. Faravelli, and E. Ranzi. Kinetic modelling of knock properties in internal combustion engines. SAE International, 2006. SAE paper 06FFL-191.

[11] J. F. Griffiths. Reduced kinetic models and their application to practical combustion systems. Progress in energy and combustion science, 21:25-207, 1995. 
[12] M. A. Marr, J.S. Wallace, S. Chandra, L. Pershin, and J. Mostaghimi. A fast response thermocouple for internal combustion engine surface temperature measurements. Experimental thermal and fluid science, 34:183-189, 2009.

[13] A. Swarts. Insights relating to octane rating and the underlying role of autoignition. $\mathrm{PhD}$ thesis, University of Cape Town, February 2006.

[14] C. Viljoen. Assessment of Discrete Strategies For Octane Prediction. PhD thesis, University of Cape Town, August 2009.

[15] A. By, B. Kempinski, and J. M. Rife. Knock in spark ignition engines. SAE International, (SAE paper 810147), 1981.

[16] http://www.dresserwaukesha.com/index.cfm/go/list-products/productline/CFR-f1-f2octane-category/, April 2011.

[17] M. Perumal. A multi-zone model of the CFR engine: Investigating cascading autoignition and octane rating. Master's thesis, University of Cape Town, January 2011.

[18] J. B. Heywood. Internal combustion engine fundamentals. McGraw-Hill, international edition, 1988. Chapter 14.

[19] J. B. Heywood. Internal combustion engine fundamentals. McGraw-Hill, international edition, 1988. Appendix B.

[20] R. Meyer and J. B. Heywood. Evaporation of in-cylinder fuel droplets in an SI engine: A diagnostic-based modelling study. SAE International, 1999. SAE paper 1999-01-0567.

[21] M. Perumal and G. Floweday. An investigation of cascading autoignition and octane number using a multi-zone model of the CFR engine. SAE International, 2011. SAE paper 2011-01-0850.

$[22]$ G. Floweday. A new functional global autoignition model for hydrocarbon fuels - part 2 of 2: model formulation, development and performance assessment. 2010 SAE International powertrains, fuels and lubricants meeting, 2010. SAE paper 2010-01-2169.

[23] W. A. Sirignano. Fluid dynamics and transport of droplets and sprays. Cambridge university press, second edition, 2010.

[24] S. S. Sazhin. Advanced models of fuel droplet heating and evaporation. Progress in energy and combustion science, 32:162-214, 2006.

[25] S. S. Sazhin, T. Kristyadi, and M. R. Heikal. Fuel droplet heating and evaporation: analysis of liquid and gas phase models. SAE International, 2007. SAE paper 2007-010019 . 
[26] S. S. Sazhin, W. A. Abdelghaffar, E. M. Sazhina, and M. R. Heikal. Models of droplet transient heating: Effects on droplet evaporation, ignition, and break-up. International journal of thermal sciences, 44:610-622, 2005.

[27] S. S. Sazhin, W. A. Abdelghaffar, E. M. Sazhina, and M.R. Heikal. New approaches to numerical modeling of droplet transient heating and evaporation. International journal of heat and mass transfer, 48:4215-4228, 2005.

[28] R. Rioboo, C. Tropea, and M. Marengo. Outcome from a drop impact on solid surfaces. Atomization and sprays, 11, 2001.

[29] G. E. Cossali, M. Marengo, and M. Santini. Impact of single and multiple drop array on a liquid film. Proceedings of the 19th annual conference on liquid atomization and spray systems ILASS, 2004.

[30] P. J. O'Rourke and A. A. Amsden. A particle numerical model for wall film dynamics in port-injected engines. SAE International, 1996. SAE paper 969161.

[31] A. L. N. Moreira, A. S. Moita, and M. R. Panao. Advances and challenges in explaining fuel spray impingement: How much of single droplet impact research is useful? Progress in energy and combustion science, 6:554-580, 2010.

[32] C. Crowe, M. Sommerfeld, and Y. Tsuji. Multiphase flows with droplets and particles. CRC Press, 1998.

[33] ANSYS 12.0. FLUENT $T^{T M}$ theory guide Chapter 15: Discrete phase, January 2009.

[34] http://webbook.nist.gov/chemistry/fluid/, April 2010.

[35] B. Poling, J. M. Prausnitz, and J. P. O'Connel. The properties of gases and liquids. McGraw-Hill, 2001. Chapter 11.

[36] L. P. Wyszynski, C. R. Stone, and G. T. Kalghatgi. The volumetric efficiency of direct and port injected gasoline engines with different fuels. SAE International, 2002. SAE paper 2002-01-0839.

[37] B. Poling, J. M. Prausnitz, and J. P. O'Connel. The properties of gases and liquids. McGraw-Hill, 2001. Chapter 7.

[38] R. Stauch and U. Maas. The auto-ignition of single n-heptane/iso-octane droplets. International journal of heat and mass transfer, 50:3047-3053, 2006.

[39] D. Bradley and R. A. Head. Engine autoignition: The relationship between octane numbers and autoignition delay times. Combustion and flame, 147:171-184, 2006. 
[40] C. Chen, D.R. Amlee, R. J. R. Johns, and Y. Zeng. Detailed modeling of liquid fuel sprays in one-dimensional gas flow simulation. SAE International, 2004. SAE paper 2004-21-3000.

[41] T. P. Hsiao. Flame propagation model for the cfr engine under knocking and non-knocking conditions. Master's thesis, University of Cape Town, March 2006.

[42] C. Liu and G.A. Karim. A 3d-simulation with detailed chemical kinetics of combustion and quenching in an hcci engine. SAE International, 2008. SAE paper 2008-01-1655.

[43] R. Meyer and J. B. Heywood. Liquid fuel transport into the cylinder of a firing portinjected SI engine during start up. SAE Transactions, (SAE paper 970865), 1997.

[44] Introductory GAMBIT $T^{T M}$ Training. FLUENT ${ }^{\mathrm{TM}} 6.3$ Online Training, www.fluentusers.com.

[45] GAMBIT ${ }^{T M}$ 2.4 Documentation. Section 3.3.1.

[46] ANSYS 12.0. FLUENT $T^{T M}$ user guide Chapter 31: Field function definitions, January 2009 .

[47] H. K. Versteeg and W. Malalasekera. An Introduction to Computational Fluid Dynamics, the FVM Chapter 2: Conservation laws of fluid motion. Pearson Prentice Hall, 2007.

[48] H. K. Versteeg and W. Malalasekera. An Introduction to Computational Fluid Dynamics, the FVM Chapter 1: Introduction. Pearson Prentice Hall, 2007.

[49] ANSYS 12.0. FLUENT ${ }^{T M}$ theory guide Chapter 4: Turbulence, January 2009.

[50] H.K. Versteeg and W. Malalasekera. An Introduction to Computational Fluid Dynamics, the FVM Chapter 3: Turbulence and its modelling. Pearson Prentice Hall, second edition, 2007.

[51] Modelling Turbulent Flows. FLUENT ${ }^{\mathrm{TM}}$ introductory training.

[52] S. Orsino. Ansys presentation: Best practice for gas combustion (non-premixed model) in fluent ${ }^{\mathrm{TM}}$.

[53] V. Bertram. Practical ship hydrodynamics Chapter 1. Butterworth-Heinemann, 2000.

[54] http://www.cortana.com/20061218-boundary-layer-of-flat-plate-jpg, October 2008.

[55] H. K. Versteeg and W. Malalasekera. An Introduction to Computational Fluid Dynamics, the FVM Chapter 5: Finite volume method for convection-diffusion problems. Pearson Prentice Hall, 2007. 
[56] ANSYS 12.0. FLUENT ${ }^{T M}$ theory guide Chapter 18: Solver theory, January 2009.

[57] H.K. Versteeg and W. Malalasekera. An Introduction to Computational Fluid Dynamics, the FVM Chapter 6: Algorithms for pressure-velocity coupling. Pearson Prentice Hall, second edition, 2007. Chapter 6.

[58] H.K. Versteeg and W. Malalasekera. An Introduction to Computational Fluid Dynamics, the FVM Chapter 8: The FVM for unsteady flows. Pearson Prentice Hall, second edition, 2007.

[59] P. Tucker and A. Mosquera. Introduction to grid and mesh generation for CFD. The international association for the engineering analysis community.

[60] L. F. Richardson. The approximate arithmetical solution by finite differences of physical problems involving differential equations, with an application to the stresses in a masonry dam. Transactions of the Royal Society of London, Ser, A, 210, 1910.

[61] L. F. Richardson and J. A. Gaunt. The deferred approach to the limit. Transactions of the Royal Society of London, Ser, A, 226, 1927.

[62] I. B. Celik. Procedure for estimation and reporting of discretization error in CFD applications. Mechanical and aerospace engineering department of West Virginia University.

[63] ANSYS 12.0. FLUENT TM user guide Chapter 23: Modeling the discrete phase, January 2009.

[64] ANSYS 12.0. FLUENT ${ }^{T M}$ user guide Chapter 11: Modeling flows using sliding and deforming meshes, January 2009.

[65] J. B. Heywood. Internal combustion engine fundamentals. McGraw-Hill, international edition, 1988. Chapter 2.

[66] ANSYS 12.0. FLUENT $T^{T M}$ user guide Chapter 12: Modeling turbulence, January 2009.

[67] ANSYS 12.0. FLUENT $T^{T M}$ user guide Chapter 15: Modeling species transport and finiterate chemistry, January 2009.

[68] ANSYS 12.0. FLUENT TM user guide Chapter 26: Using the solver, January 2009. 